\title{
The burden of disease in Spain: results from the global burden of disease study 2010
}

\author{
Josep Maria Haro ${ }^{1,2^{*}}$, Stefanos Tyrovolas ${ }^{1,2}$, Noe Garin ${ }^{1,2}$, Cesar Diaz-Torne ${ }^{3}$, Loreto Carmona ${ }^{4}$, \\ Lidia Sanchez-Riera ${ }^{5,6}$, Fernando Perez-Ruiz ${ }^{7}$ and Christopher JL Murray ${ }^{8}$
}

\begin{abstract}
Background: We herein evaluate the Spanish population s trends in health burden by comparing results of two Global Burden of Diseases, Injuries, and Risk Factors Studies (the GBD studies) performed 20 years apart.

Methods: Data is part of the GBD study for 1990 and 2010. We present results for mortality, years of life lost (YLLs), years lived with disability, and disability-adjusted life years (DALYs) for the Spanish population. Uncertainty intervals for all measures have been estimated.

Results: Non-communicable diseases accounted for 3,703,400 (95\% Cl 3,648,270 3,766,720) (91.3\%) of 4,057,400 total deaths, in the Spanish population. Cardiovascular and circulatory diseases were the main cause of mortality among non-communicable diseases (34.7\% of total deaths), followed by neoplasms (27.1\% of total deaths). Neoplasms, cardiovascular and circulatory diseases, and chronic respiratory diseases were the top three leading causes for YLLs. The most important causes of DALYs in 2010 were neoplasms, cardiovascular and circulatory diseases, musculoskeletal disorders, and mental and behavioral disorders.

Conclusions: Mortality and disability in Spain have become even more linked to non-communicable diseases over the last years, following the worldwide trends. Cardiovascular and circulatory diseases, neoplasms, mental and behavioral disorders, and neurological disorders are the leading causes of mortality and disability. Specific focus is needed from health care providers and policy makers to develop health promotion and health education programs directed towards non-communicable disorders.
\end{abstract}

Keywords: Disability-adjusted life years, Global Burden of Diseases, Injuries, and Risk Factors Studies, Spain, Mortality, Years lived with disability, Years of life lost

\section{Background}

The impact of diseases and injures on population health is usually assessed with measures of mortality and non-fatal health outcomes [1]. These estimates are used to signal the most relevant public health problems, allow comparison between different populations and different health conditions, and assess changes over time. The only comprehensive effort to date to estimate summary measures of the global population health, by cause and by world

\footnotetext{
* Correspondence: jmharo@pssjd.org

${ }^{1}$ Parc Sanitari Sant Joan de Du, Universitat de Barcelona, Fundaci Sant Joan de Du. Dr Antoni Pujades, 42, 08830 Sant Boi de Llobregat, Barcelona, Spain

${ }^{2}$ Instituto de Salud Carlos III, Centro de Investigacin Biomdica en Red de Salud Mental CIBERSAM, Dr. Esquerdo 46, 28007 Madrid, Spain

Full list of author information is available at the end of the article
}

region, is the ongoing Global Burden of Diseases, Injuries, and Risk Factors (GBD) initiative [2,3].

The first GBD study analyzed data from 1990 [4] and was published in 1993. Since then, a number of updates have been published [5]. The Global Burden of Diseases, Injuries, and Risk Factors Study 2010 (GBD 2010) has updated and expanded previous efforts to include 1,160 diseases and injury sequelae from the previous analysis, which included 483 diseases. The most important limitation of previous GBD studies is that results were not estimated with uncertainty [6]. Specifically, uncertainty can come from many sources, including heterogeneity in the empirical data that are available and uncertainty in the indirect estimation models used to make predictions for populations with little or no data. However, this 
limitation has been solved in the recent analysis of the GBD 2010 study.

The GBD initiative uses disability-adjusted life years (DALYs) and mortality as the summary measurements of the impact of health conditions on population health. The DALY combines the years of life lost (YLLs) due to premature mortality and the years lived with disability (YLDs). The use of DALYs as measure of impact, the inclusion of a large number of communicable and non-communicable conditions, the analysis of the impact of health conditions stratified by gender and age, and the effort to use comparable methodologies across countries and regions make the GBD project an excellent tool to understand the determinants of health and their variability across time and regions. According to previous reported global results, in 1990, $47 \%$ of DALYs were attributed to communicable, maternal, neonatal, and nutritional disorders, $43 \%$ to noncommunicable diseases, and $10 \%$ to injuries, while in 2010 , this had reversed to $35 \%, 54 \%$, and $11 \%$, respectively [5]. Besides clear differences among countries with different socioeconomic conditions, heterogeneity is also present when analyzing and comparing countries with more similar socioeconomic conditions. For example, in the United Kingdom (UK), mortality and disability as well as overall health has improved in absolute terms between 1990 and 2010. However, according to Murray et al. [7], the UK performed worse than other EU countries in age-standardized mortality, YLLs, and life expectancy rates. The UK age-standardized DALY rates for chronic obstructive pulmonary disease, disorders of drug use, lower respiratory infections, breast cancers, and preterm birth complications were significantly higher compared to the mean of the EU-15 area countries [7].

Spain is a southern European country with distinct sociodemographic and health characteristics; while in former years large proportions of the population adhered to a Mediterranean diet, dietary habits are changing rapidly with alarming rates of obesity and smoking and alcohol drinking are still highly prevalent in the country despite public health efforts to reduce consumption. Nevertheless, the, until recently, universal coverage of the National Health System also facilitates good health care levels and the implementation of some preventive actions. Until now, to the best of our knowledge, only two studies have tried to assess the burden of disease in Spain. Genova-Maleras et al. [8] estimated the impact of different diseases using DALYs. According to the researchers, the DALYs due to all diseases and injuries were estimated at 5.1 million in Spain. Almost 90\% of these were attributed to chronic diseases. Specifically, the leading causes of DALYs were neurological and mental disorders, followed by malignant neoplasms and cardiovascular diseases [8]. However, the aforementioned results were partially limited, since the mortality data came from Spanish registers, while the incidence and severity rates were obtained from estimations of the WHO for a variety of European countries (i.e., WHO European Region Eur-A). A more recent project, but only including data from Valencia, revealed similar results [9], reporting that the number of DALYs gradually increased with age and almost $27 \%$ of all DALYs occurred among people over the age of 70 [9].

Given the scarcity of analysis of the data from Spain and the relevance of learning from the experience and comparison with similar and not so similar countries, the aim of the present work was to evaluate the change in burden of disease in Spain, comparing the available data of the GBD over time, between 1990 and 2010.

\section{Methods}

\section{Overview}

The GBD 2010 study estimates the impact of 291 diseases and injuries and 67 risk factors for 187 countries distributed into 21 world regions between 1990 and 2010. For each cause, 1 to 24 sequelae were defined. Sequelae are the clinical outcomes that can be related to specific diseases and injuries such as neuropathy due to diabetes. In total, the study includes 1,160 sequelae. More detailed information about data and analysis for the GBD 2010 have been previously reported [2,5,6,10,11]. For the present analysis, only information regarding Spain will be reported.

\section{Measurements}

We report data on mortality, YLLs due to premature mortality, YLDs, and DALYs. Age-specific mortality rates for Spain were estimated for each sex. As in other developed countries, information about deaths was predominantly driven by data from official vital registration systems [12]. The denominators were based on Spanish census returns and intercensal estimates. Similarly to the other 187 countries of the GBD study, the estimated number of deaths and YLLs was based on 235 defined underlying causes of mortality from the list of 291 diseases and injuries, for 20 age groups and both sexes [2,13]. The YLLs were computed by multiplying the number of deaths in each age group by a reference life expectancy [2]. The YLDs were computed by multiplying the prevalence of a sequelae by its disability weight (DW), used to quantify population s health losses. This procedure varies slightly compared to previous GBD studies, in which incidence and average duration of the case until remission was used instead of prevalence [14]. A total of 1,160 possible sequelae of diseases and injuries were analyzed. Murray et al. [5] have described the systematic analysis of available data conducted for each sequelae with regard to the prevalence, incidence, remission, and excess mortality. For each age-sex-year group, estimates were made for most sequelae using Bayesian meta-regression methods. DWs were obtained 
for 220 health states covering the 1,160 sequelae [6]. For each sequelae, DWs were derived based on the scoring of short lay descriptions of the relevant health domains in large population-based studies in several countries (i.e., Peru, USA) and through an open internet survey [15]. Finally, for the estimation of the DALYs, the arithmetic sum of YLLs and YLDs was used.

The YLDs age-standardized rates for each cause, in 1990 and 2010, were calculated. For this procedure the WHO age-standard was used as has been described in former GBD analyses [16]. Spain s YLDs age-standardized rankings were compared with other European countries. The aforementioned comparison of YLDs age-standardized rates provides an opportunity to compare the YLDs across the European countries in specific periods, controlling for number variations and crude rates due to differences in population age.

In order to differentiate the change in DALYs due to demographic variations from those to health changes or other reasons, two counterfactual increases in total DALYs were calculated based on the 1990 population sex and age distribution and compared with the observed increase from 1990 to 2010: i) the expected increase in total DALYs if total population increase had been as observed but without change in the age/sex structure or in strata-specific DALY rates; ii) the expected increase in total DALYs if the population and its age/sex structure had changed as observed but without change in strata-specific DALY rates (application of 1990 stratum-specific DALY rates to the 2010 population strata sizes). The first estimate, (i), provides the increase attributable to population increase without population aging; (ii) minus (i) gives the increase attributable to population aging and the observed increase from 1990 to 2010 minus (ii) gives the increase attributable to changes in stratum-specific DALY rates.

\section{Uncertainty levels}

Uncertainty levels for mortality rate were estimated using standard simulation methods [2]. Uncertainty for mortality and YLLs reflected uncertainty in the levels of all-cause mortality and uncertainty in the estimation of each mortality cause, in each age group, sex, and year. Uncertainty in the disability weight for each sequelae was propagated into the estimates of YLDs for each disease and injury. For a more accurate estimation of YLDs, the effect of comorbidity was taken into account, as explained in Vos et al. [10]. Specifically, the procedure of microsimulation for each country (explicitly here for Spain), age, sex, and year were used in a large number of simulated individuals. This standard simulation method was repeated 1,000 times to be able to capture uncertainty in the prevalence of all sequelae and disability weights [10].

\section{Results}

From 1990 to 2010, the overall Spanish population increased by almost $15 \%$ (from 38,914,907 to 44,558,264 people) (Table 1). A similar increase was observed in males and females, close to $15 \%$ and $14 \%$, respectively. The population of older adults in Spain presented the highest increase among all age groups. For example, in octogenarians (aged 80+ years), the increase was almost double. The highest decrease of population was observed in the younger ages (0 20 years old), where the population dropped by $21 \%$.

Table 2 illustrates the main causes of mortality and YLLs by gender and age group for the Spanish population. As expected, non-communicable diseases were the major cause of mortality, accounting for 3,703,400 (95\% CI $3,648,2703,766,720$ ) (91.3\%) of 4,057,400 total deaths. Cardiovascular and circulatory diseases were the main cause of mortality among the non-communicable diseases (34.7\% of total deaths), followed by neoplasms (27.1\% of total deaths). The third category, injuries, accounted for $4.1 \%$ of Spanish deaths [170,040, 95\% CI $(148,350$ 187,710)].

Age-specific analysis revealed that non-communicable diseases remained the major cause of mortality except for children below 1 year of age. Cardiovascular- and circulatory-related mortality raised with ageing, from $6 \%$ of deaths in individuals younger than 20 years old to almost $41 \%$ in those 80 years old and over. Neoplasmrelated mortality was almost $22 \%$ of deaths in individuals aged up to 40 years, nearly doubled in the group aged 4079 years and finally dropped to $17 \%$ in the group of octogenarians. On the contrary, the main cause of mortality in newborns was communicable diseases, which accounted for $61.5 \%$ of all deaths within the group.

In regard to gender differences, the main cause of male mortality was neoplasms (33\%), followed by cardiovascular diseases $(30.3 \%)$, chronic respiratory diseases $(9.5 \%)$, neurological diseases (5.3\%), and injuries (5.2\%). On the other hand, the main cause of female mortality were cardiovascular diseases (39.4\%) followed by neoplasms (21.4\%), neurological disorders (9.4\%), and the group formed by

Table 1 Spanish population (millions) in 1990 and 2010

\begin{tabular}{cllr}
\hline & $\mathbf{1 9 9 0}$ & $\mathbf{2 0 1 0}$ & $\boldsymbol{\Delta} \%$ \\
\hline Both sexes, all ages & $38,914,907$ & $44,558,264$ & 15 \\
0 19 years old & $11,078,521$ & $8,781,618$ & -21 \\
20 39 years old & $11,618,044$ & $13,004,297$ & 12 \\
40 59 years old & $8,820,927$ & $12,589,546$ & 43 \\
6079 years old & $6,240,651$ & $7,878,252$ & 26 \\
$80+$ years old & $1,156,764$ & $2,304,554$ & 99 \\
Males, all ages & $19,057,590$ & $21,964,405$ & 15 \\
Females, all ages & $19,857,317$ & $22,593,859$ & 14 \\
\hline
\end{tabular}


Table 2 Main causes of mortality and years of life lost (YLLs) by age groups and by gender, for the Spanish population in 2010

\begin{tabular}{|c|c|c|c|c|c|c|c|c|c|c|c|c|}
\hline \multirow[t]{2}{*}{ Deaths (hundreds) } & \multirow[b]{2}{*}{ Both sexes, all ages } & \multirow[b]{2}{*}{$\begin{array}{l}<1 \\
\text { year }\end{array}$} & \multirow[b]{2}{*}{$\begin{array}{l}119 \\
\text { years }\end{array}$} & \multirow[b]{2}{*}{$\begin{array}{l}2039 \\
\text { years }\end{array}$} & \multirow[b]{2}{*}{$\begin{array}{l}4059 \\
\text { years }\end{array}$} & \multirow[b]{2}{*}{$\begin{array}{l}6079 \\
\text { years }\end{array}$} & \multirow[b]{2}{*}{$\begin{array}{l}80+ \\
\text { years }\end{array}$} & \multirow[b]{2}{*}{ Males, all ages } & \multirow[b]{2}{*}{ Females, all ages } & \multicolumn{3}{|l|}{ YLLs (hundreds) } \\
\hline & & & & & & & & & & Both sexes, all ages & Males, all ages & Females, all ages \\
\hline All causes & $4,057.4$ & 16.9 & 14.9 & 74.9 & 345.6 & $1,283.0$ & $2,322.2$ & $2,049.9$ & $2,007.5$ & $54,562.8$ & $32,736.3$ & $21,826.5$ \\
\hline $\begin{array}{l}\text { Communicable, maternal, } \\
\text { neonatal, and nutritional } \\
\text { disorders }\end{array}$ & $\begin{array}{l}183.96 \\
(157.53214 .88)\end{array}$ & 10.4 & 1.3 & 5.8 & 14.3 & 35.6 & 116.6 & $\begin{array}{l}89.84 \\
(74.84 \text { 110.39) }\end{array}$ & $\begin{array}{l}94.12 \\
(75.54 \quad 117)\end{array}$ & $\begin{array}{l}3,124.9 \\
(2,873.32 \quad 3,397.56)\end{array}$ & $\begin{array}{ll}1,818.74 & \\
(1,629.9 & 2,038.1)\end{array}$ & $\begin{array}{ll}1,306.17 & \\
(1,154.6 & 1,471.72)\end{array}$ \\
\hline HIV/AIDS and tuberculosis & $\begin{array}{l}16.76 \\
(14.719 .39)\end{array}$ & 0.0 & 0.0 & 3.6 & 7.5 & 3.0 & 2.8 & $\begin{array}{l}12.12 \\
(10.2114 .78)\end{array}$ & $\begin{array}{l}4.63 \\
(3.855 .63)\end{array}$ & $\begin{array}{l}555.31 \\
(480.43649 .07)\end{array}$ & $\begin{array}{l}420.04 \\
(349.4512 .85)\end{array}$ & $\begin{array}{l}135.27 \\
(115.48 \text { 159.21) }\end{array}$ \\
\hline $\begin{array}{l}\text { Diarrhea, lower respiratory } \\
\text { infections, meningitis, and } \\
\text { other common infectious } \\
\text { diseases }\end{array}$ & $\begin{array}{l}138.1 \\
(113.55169 .75)\end{array}$ & 0.4 & 0.7 & 1.5 & 5.2 & 27.8 & 102.4 & $\begin{array}{l}64.36 \\
(49.585 .36)\end{array}$ & $\begin{array}{l}73.75 \\
(55.79 \text { 96.84) }\end{array}$ & $\begin{array}{ll}1,432.97 & \\
(1,244.82 & 1,680.97)\end{array}$ & $\begin{array}{l}783.48 \\
(640.77986 .07)\end{array}$ & $\begin{array}{l}649.49 \\
(534.56788 .48)\end{array}$ \\
\hline $\begin{array}{l}\text { Neglected tropical diseases } \\
\text { and malaria }\end{array}$ & $\begin{array}{l}1.66 \\
(0.843 .19)\end{array}$ & 0.0 & 0.0 & 0.0 & 0.0 & 0.3 & 0.5 & $\begin{array}{l}0.62 \\
(0.311 .14)\end{array}$ & $\begin{array}{l}1.04 \\
(0.42 .45)\end{array}$ & $\begin{array}{l}49.34 \\
(27.4789 .06)\end{array}$ & $\begin{array}{l}20.64 \\
(11.3536 .68)\end{array}$ & $\begin{array}{l}28.7 \\
(11.8561 .85)\end{array}$ \\
\hline Maternal disorders & $\begin{array}{ll}0.32 & \\
(0.22 & 0.42)\end{array}$ & 0.0 & 0.0 & 0.0 & 0.0 & 0.0 & 0.0 & * & $\begin{array}{ll}0.32 & \\
(0.22 & 0.42)\end{array}$ & $\begin{array}{l}16.2 \\
(10.8621 .52)\end{array}$ & * & $\begin{array}{l}16.2 \\
(10.8621 .52)\end{array}$ \\
\hline Neonatal disorders & $\begin{array}{l}9.8 \\
(8.5411 .03)\end{array}$ & 9.8 & 0.0 & 0.0 & 0.0 & 0.0 & 0.0 & $\begin{array}{ll}5.5 \\
(4.636 .4)\end{array}$ & $\begin{array}{l}4.3 \\
(3.45 .11)\end{array}$ & $\begin{array}{l}842.8 \\
(734.44948 .51)\end{array}$ & $\begin{array}{l}473.06 \\
(398.16550 .48)\end{array}$ & $\begin{array}{l}369.74 \\
(292.36439 .4)\end{array}$ \\
\hline Nutritional deficiencies & $\begin{array}{l}9.52 \\
(5.2413 .19)\end{array}$ & 0.0 & 0.0 & 0.0 & 0.0 & 1.3 & 7.8 & $\begin{array}{l}3.24 \\
(1.824 .57)\end{array}$ & $\begin{array}{l}6.29 \\
(2.729 .74)\end{array}$ & $\begin{array}{l}82.92 \\
(48.12106 .53)\end{array}$ & $\begin{array}{l}35.8 \\
(20.245 .91)\end{array}$ & $\begin{array}{l}47.12 \\
(22.0768 .11)\end{array}$ \\
\hline $\begin{array}{l}\text { Other communicable, maternal, } \\
\text { neonatal, and nutritional } \\
\text { disorders }\end{array}$ & $\begin{array}{l}7.79 \\
(5.079 .57)\end{array}$ & 0.0 & 0.0 & 0.1 & 1.1 & 3.1 & 3.1 & $\left.\begin{array}{ll}4 \\
(2.37 & 5.02\end{array}\right)$ & $\begin{array}{ll}3.8 & \\
(2.32 & 5.06)\end{array}$ & $\begin{array}{l}145.36 \\
(100.68 \text { 168.33) }\end{array}$ & $\begin{array}{l}85.71 \\
(56.07102 .53)\end{array}$ & $\begin{array}{l}59.64 \\
(39.6373 .75)\end{array}$ \\
\hline Non-communicable diseases & $\begin{array}{l}3,703.42 \\
(3,648.27 \quad 3,766.72)\end{array}$ & 6.1 & 7.5 & 39.6 & 297.0 & 1.205 .7 & 2.147 .2 & $\begin{array}{l}1,853.9 \\
(1,818.92 \quad 1,891.49)\end{array}$ & $\begin{array}{ll}1,849.53 & \\
(1,807.93 & 1,895.78)\end{array}$ & $\begin{array}{l}46,970.31 \\
(46,249.68 \quad 48,068.86)\end{array}$ & $\begin{array}{l}27,624.78 \\
(27,059.44 \quad 28,507.45)\end{array}$ & $\begin{array}{l}19,345.53 \\
(18,957.63 \quad 19,922.49)\end{array}$ \\
\hline Neoplasms & $\begin{array}{ll}1,101.24 & \\
(1,014.78 & 1,168.33)\end{array}$ & 0.0 & 3.2 & 16.5 & 166.4 & 513.7 & 401.3 & $\begin{array}{l}671.72 \\
(603.52728 .99)\end{array}$ & $\begin{array}{l}429.52 \\
(386.58 \text { 469.72) }\end{array}$ & $\begin{array}{ll}18,488.09 & \\
(17,216.3 & 19,429.35)\end{array}$ & $\begin{array}{ll}11,636.36 & \\
(10,542.8 & 12,486.19)\end{array}$ & $\begin{array}{l}6851.73 \\
(6324.27 \quad 7307.47)\end{array}$ \\
\hline Esophageal cancer & $\begin{array}{l}19.45 \\
(14.4126 .46)\end{array}$ & 0.0 & 0.0 & 0.0 & 4.9 & 9.9 & 4.6 & $\begin{array}{l}16.47 \\
(11.7923 .21)\end{array}$ & $\begin{array}{l}2.97 \\
(1.894 .47)\end{array}$ & $\begin{array}{l}388.59 \\
(286.84536 .01)\end{array}$ & $\begin{array}{l}349.47 \\
(253.61493 .6)\end{array}$ & $\begin{array}{l}39.11 \\
(23.4353 .73)\end{array}$ \\
\hline Stomach cancer & $\begin{array}{l}64.9 \\
(49.7293 .74)\end{array}$ & 0.0 & 0.0 & 0.7 & 7.9 & 29.2 & 27.1 & $\begin{array}{l}36.68 \\
(24.6952 .52)\end{array}$ & $\begin{array}{l}28.22 \\
(18.1351 .95)\end{array}$ & $\begin{array}{ll}986.61 & \\
(741.61 & 1,378.18)\end{array}$ & $\begin{array}{l}613.71 \\
(403.16859 .83)\end{array}$ & $\begin{array}{l}372.9 \\
(229.02636 .47)\end{array}$ \\
\hline Liver cancer & $\begin{array}{l}52.14 \\
(42.3965 .26)\end{array}$ & 0.0 & 0.0 & 0.3 & 6.8 & 26.6 & 18.2 & $\begin{array}{l}32.93 \\
(25.0942 .88)\end{array}$ & $\begin{array}{l}19.2 \\
(14.5528 .42)\end{array}$ & $\begin{array}{l}837.17 \\
(701.49 \quad 1,036.73)\end{array}$ & $\begin{array}{l}591.1 \\
(470.26768 .21)\end{array}$ & $\begin{array}{l}246.07 \\
(197.75342 .93)\end{array}$ \\
\hline Larynx cancer & $\begin{array}{l}17.38 \\
(9.0331 .26)\end{array}$ & 0.0 & 0.0 & 0.0 & 4.8 & 8.9 & 3.6 & $\begin{array}{l}16.56 \\
(8.3830 .24)\end{array}$ & $\begin{array}{l}0.83 \\
(0.381 .31)\end{array}$ & $\begin{array}{l}361.52 \\
(190.52673 .47)\end{array}$ & $\begin{array}{l}348.38 \\
(180.74659 .95)\end{array}$ & $\begin{array}{l}13.14 \\
(5.5922 .2)\end{array}$ \\
\hline $\begin{array}{l}\text { Trachea, bronchus, and lung } \\
\text { cancers }\end{array}$ & $\begin{array}{l}191.92 \\
(123.09218 .32)\end{array}$ & 0.0 & 0.0 & 1.3 & 39.2 & 105.4 & 46.0 & $\begin{array}{l}163.47 \\
(99.12188 .56)\end{array}$ & $\begin{array}{l}28.45 \\
(12.4335 .7)\end{array}$ & $\begin{array}{l}3,641.21 \\
(2,366.65 \quad 4,115.82)\end{array}$ & $\begin{array}{ll}3,088.96 & \\
(1,923.35 & 3,509.43)\end{array}$ & $\begin{array}{l}552.25 \\
(221.23698 .17)\end{array}$ \\
\hline Breast cancer & $\begin{array}{l}65.87 \\
(58.9873 .57)\end{array}$ & 0.0 & 0.0 & 2.0 & 16.6 & 24.7 & 22.5 & * & $\begin{array}{l}65.87 \\
(58.9873 .57)\end{array}$ & $\begin{array}{l}1281.35 \\
(1177.31410 .43)\end{array}$ & * & $\begin{array}{ll}1,281.35 & \\
(1,177.3 \quad 1,410.43)\end{array}$ \\
\hline Cervical cancer & $\begin{array}{l}9.09 \\
(5.9813 .94)\end{array}$ & 0.0 & 0.0 & 0.4 & 2.9 & 3.3 & 2.4 & * & $\begin{array}{l}9.09 \\
(5.9813 .94)\end{array}$ & $\begin{array}{l}207.1 \\
(130.86301 .52)\end{array}$ & * & $\begin{array}{l}207.1 \\
(130.86301 .52)\end{array}$ \\
\hline Uterine cancer & $\begin{array}{l}12.5 \\
(5.2917 .15)\end{array}$ & 0.0 & 0.0 & 0.0 & 1.2 & 6.3 & 5.0 & * & $\begin{array}{l}12.5 \\
(5.2917 .15)\end{array}$ & $\begin{array}{l}181.01 \\
(81.49256 .02)\end{array}$ & * & $\begin{array}{l}181.01 \\
(81.49256 .02)\end{array}$ \\
\hline
\end{tabular}


Table 2 Main causes of mortality and years of life lost (YLLs) by age groups and by gender, for the Spanish population in 2010 (Continued)

\begin{tabular}{|c|c|c|c|c|c|c|c|c|c|c|c|c|}
\hline Prostate cancer & $\begin{array}{ll}76.48 & \\
(43.85 & 133.33)\end{array}$ & 0.0 & 0.0 & 0.0 & 1.3 & 29.5 & 45.7 & $\begin{array}{ll}76.48 & \\
(43.85 & 133.33)\end{array}$ & * & $\begin{array}{ll}818.99 & \\
(459.29 & 1,372.44)\end{array}$ & $\begin{array}{ll}818.99 & \\
(459.29 & 1,372.44)\end{array}$ & * \\
\hline Colon and rectum cancers & $\begin{array}{l}160.31 \\
(126.24180 .29)\end{array}$ & 0.0 & 0.0 & 1.1 & 16.9 & 71.9 & 70.3 & $\begin{array}{l}90.85 \\
(66.16104 .65)\end{array}$ & $\begin{array}{l}69.46 \\
(50.3982 .73)\end{array}$ & $\begin{array}{l}2,323.71 \\
(1,897.63 \quad 2,571.55)\end{array}$ & $\begin{array}{ll}1,399.22 & \\
(1,060.67 & 1,580.73)\end{array}$ & $\begin{array}{l}924.49 \\
(704.14 \\
1,098.13)\end{array}$ \\
\hline Mouth cancer & $\begin{array}{l}13.87 \\
(11.6216 .79)\end{array}$ & 0.0 & 0.0 & 0.1 & 3.7 & 6.2 & 3.6 & $\begin{array}{l}10.33 \\
(8.4813 .11)\end{array}$ & $\begin{array}{l}3.54 \\
(2.144 .85)\end{array}$ & $\begin{array}{l}282.8 \\
(236.88 \text { 352.29) }\end{array}$ & $\begin{array}{l}230.16 \\
(187.58298 .5)\end{array}$ & $\begin{array}{l}52.65 \\
(31.3470 .41)\end{array}$ \\
\hline Nasopharynx cancer & $\begin{array}{l}2.61 \\
(1.643 .53)\end{array}$ & 0.0 & 0.0 & 0.0 & 0.8 & 1.2 & 0.5 & $\begin{array}{l}1.96 \\
(1.142 .91)\end{array}$ & $\begin{array}{l}0.65 \\
(0.311 .05)\end{array}$ & $\begin{array}{l}62.2 \\
(39.43 \\
87.48)\end{array}$ & $\begin{array}{l}49.32 \\
(29.1274 .66)\end{array}$ & $\begin{array}{l}12.88 \\
(6.1419 .83)\end{array}$ \\
\hline $\begin{array}{l}\text { Cancer of other part of } \\
\text { pharynx and oropharynx }\end{array}$ & $\begin{array}{l}8.63 \\
(4.9311 .43)\end{array}$ & 0.0 & 0.0 & 0.0 & 3.2 & 4.1 & 1.2 & $\begin{array}{l}7.5 \\
(4.1210 .7)\end{array}$ & $\begin{array}{ll}1.13 & \\
(0.48 & 1.83)\end{array}$ & $\begin{array}{l}206.07 \\
(118.56292 .36)\end{array}$ & $\begin{array}{l}185.2 \\
(104.09277 .98)\end{array}$ & $\begin{array}{l}20.87 \\
(8.5233 .12)\end{array}$ \\
\hline $\begin{array}{l}\text { Gallbladder and biliary tract } \\
\text { cancer }\end{array}$ & $\begin{array}{l}19.93 \\
(13.6130 .72)\end{array}$ & 0.0 & 0.0 & 0.0 & 1.4 & 9.3 & 9.0 & $\begin{array}{l}7.04 \\
(4.2211 .03)\end{array}$ & $\begin{array}{l}12.89 \\
(7.1422 .96)\end{array}$ & $\begin{array}{l}269.16 \\
(187.54408 .35)\end{array}$ & $\begin{array}{l}107.66 \\
(65.4163 .82)\end{array}$ & $\begin{array}{l}161.5 \\
(88.78288 .73)\end{array}$ \\
\hline Pancreatic cancer & $\begin{array}{l}53.44 \\
(38.1770 .44)\end{array}$ & 0.0 & 0.0 & 0.3 & 7.6 & 27.4 & 18.0 & $\begin{array}{l}27.66 \\
(17.7936 .7)\end{array}$ & $\begin{array}{l}25.78 \\
(15.5639 .61)\end{array}$ & $\begin{array}{l}875.5 \\
(637.54 \\
1,113.72)\end{array}$ & $\begin{array}{l}517.94 \\
(335.01680 .03)\end{array}$ & $\begin{array}{l}357.56 \\
(215.21544 .31)\end{array}$ \\
\hline Malignant melanoma of skin & $\begin{array}{l}9.36 \\
(5.5813 .09)\end{array}$ & 0.0 & 0.0 & 0.5 & 2.2 & 3.8 & 2.7 & $\begin{array}{l}5.14 \\
(2.28 \text { 8.31) }\end{array}$ & $\begin{array}{l}4.22 \\
(2.236 .97)\end{array}$ & $\begin{array}{l}198.46 \\
(125.2285 .93)\end{array}$ & $\begin{array}{l}114.49 \\
(55.15197 .21)\end{array}$ & $\begin{array}{l}83.97 \\
(46.36131 .3)\end{array}$ \\
\hline Non-melanoma skin cancer & $\begin{array}{l}6.79 \\
(4.1211 .36)\end{array}$ & 0.0 & 0.0 & 0.0 & 0.3 & 1.6 & 4.7 & $\begin{array}{l}3.57 \\
(1.726 .74)\end{array}$ & $\begin{array}{l}3.23 \\
(1.427 .1)\end{array}$ & $\begin{array}{l}72.93 \\
(44.47\end{array}$ & $\begin{array}{l}46.17 \\
(22.3590 .37)\end{array}$ & $\begin{array}{l}26.75 \\
(11.8456 .5)\end{array}$ \\
\hline Ovarian cancer & $\begin{array}{l}21.15 \\
(13.3527 .17)\end{array}$ & 0.0 & 0.0 & 0.4 & 5.0 & 10.1 & 5.6 & * & $\begin{array}{l}21.15 \\
(13.3527 .17)\end{array}$ & $\begin{array}{l}415.83 \\
(277.13553 .19)\end{array}$ & * & $\begin{array}{l}415.83 \\
(277.13553 .19)\end{array}$ \\
\hline Testicular cancer & $\begin{array}{l}0.66 \\
(0.321 .1)\end{array}$ & 0.0 & 0.0 & 0.0 & 0.0 & 0.0 & 0.1 & $\begin{array}{l}0.66 \\
(0.321 .1)\end{array}$ & * & $\left.\begin{array}{ll}21.3 & \\
(11.38 & 31.3\end{array}\right)$ & $\begin{array}{l}21.3 \\
(11.38 \quad 31.3)\end{array}$ & * \\
\hline $\begin{array}{l}\text { Kidney and other urinary } \\
\text { organ cancers }\end{array}$ & $\begin{array}{l}34.18 \\
(24.5756 .41)\end{array}$ & 0.0 & 0.0 & 0.2 & 5.2 & 16.8 & 11.8 & $\begin{array}{l}21.44 \\
(14.9841 .31)\end{array}$ & $\begin{array}{l}12.74 \\
(7.0123 .93)\end{array}$ & $\begin{array}{l}574.91 \\
(420.5941 .19)\end{array}$ & $\begin{array}{l}385.7 \\
(278.74736 .03)\end{array}$ & $\begin{array}{l}189.21 \\
(108.55335 .81)\end{array}$ \\
\hline Bladder cancer & $\begin{array}{l}49.77 \\
(34.660 .6)\end{array}$ & 0.0 & 0.0 & 0.0 & 3.6 & 22.1 & 24.1 & $\begin{array}{l}39.86 \\
(24.750 .26)\end{array}$ & $\begin{array}{l}9.91 \\
(7.1313)\end{array}$ & $\begin{array}{l}654.29 \\
(461.6771 .51)\end{array}$ & $\begin{array}{l}549.7 \\
(357.15673 .67)\end{array}$ & $\begin{array}{l}104.6 \\
(77.76128 .75)\end{array}$ \\
\hline $\begin{array}{l}\text { Brain and nervous system } \\
\text { cancers }\end{array}$ & $\begin{array}{l}35.98 \\
(19.7547 .79)\end{array}$ & 0.0 & 0.9 & 2.0 & 8.4 & 17.2 & 7.5 & $\begin{array}{l}19.01 \\
(7.6326 .36)\end{array}$ & $\begin{array}{l}16.96 \\
(6.6325 .86)\end{array}$ & $\begin{array}{l}830.9 \\
(476.65 \quad 1,032.05)\end{array}$ & $\begin{array}{l}470.77 \\
(201.2635 .62)\end{array}$ & $\begin{array}{l}360.13 \\
(155.29483 .64)\end{array}$ \\
\hline Thyroid cancer & $\begin{array}{l}3.59 \\
(2.594 .71)\end{array}$ & 0.0 & 0.0 & 0.0 & 0.3 & 1.7 & 1.4 & $\begin{array}{ll}1.2 & \\
(0.67 & 1.73)\end{array}$ & $\begin{array}{l}2.39 \\
(1.48 \\
(.39)\end{array}$ & $\begin{array}{l}57.39 \\
(42.1973 .87)\end{array}$ & $\begin{array}{l}23.49 \\
(12.6833 .42)\end{array}$ & $\begin{array}{l}33.91 \\
(21.2847 .35)\end{array}$ \\
\hline Hodgkin's disease & $\begin{array}{l}2.65 \\
\left(\begin{array}{ll}1.69 & 3.95)\end{array}\right.\end{array}$ & 0.0 & 0.0 & 0.3 & 0.4 & 1.1 & 0.6 & $\begin{array}{l}1.43 \\
\left(\begin{array}{ll}0.72 & 2.35\end{array}\right)\end{array}$ & $\begin{array}{l}1.22 \\
\left(\begin{array}{ll}0.6 & 2.06\end{array}\right)\end{array}$ & $\begin{array}{l}70.16 \\
(46.16106 .97)\end{array}$ & $\begin{array}{l}41.58 \\
(21.5369 .86)\end{array}$ & $\begin{array}{l}28.59 \\
(14.8649 .59)\end{array}$ \\
\hline Non-Hodgkin lymphoma & $\begin{array}{l}27.11 \\
(17.9133 .76)\end{array}$ & 0.0 & 0.0 & 1.0 & 4.0 & 12.6 & 9.3 & $\begin{array}{l}14.12 \\
(8.5717 .92)\end{array}$ & $\begin{array}{l}12.98 \\
\left(\begin{array}{ll}6.48 & 17.22)\end{array}\right.\end{array}$ & $\begin{array}{l}487.31 \\
(353.12563 .21)\end{array}$ & $\begin{array}{l}283.95 \\
(184.07336 .15)\end{array}$ & $\begin{array}{l}203.35 \\
(113.58 \text { 246.19) }\end{array}$ \\
\hline Multiple myeloma & $\begin{array}{l}17.81 \\
(11.2425 .25)\end{array}$ & 0.0 & 0.0 & 0.0 & 1.6 & 9.7 & 6.4 & $\begin{array}{l}8.93 \\
(4.615 .05)\end{array}$ & $\begin{array}{l}8.88 \\
(4.4914 .08)\end{array}$ & $\begin{array}{l}267.24 \\
(175.37381 .15)\end{array}$ & $\begin{array}{l}142.53 \\
(75.4242 .08)\end{array}$ & $\begin{array}{l}124.71 \\
(64.53196 .93)\end{array}$ \\
\hline Leukemia & $\begin{array}{l}39.1 \\
(27.6448 .47)\end{array}$ & 0.0 & 0.9 & 1.5 & 3.8 & 16.3 & 16.6 & $\begin{array}{l}22.79 \\
(13.6428 .19)\end{array}$ & $\begin{array}{l}16.31 \\
(8.2523 .37)\end{array}$ & $\begin{array}{l}672.27 \\
(529803.1)\end{array}$ & $\begin{array}{l}406.11 \\
(272.07491 .1)\end{array}$ & $\begin{array}{l}266.16 \\
(149.02354 .36)\end{array}$ \\
\hline Other neoplasms & $\begin{array}{l}84.59 \\
(64.71111 .97)\end{array}$ & 0.0 & 0.8 & 2.2 & 11.6 & 37.0 & 32.9 & $\begin{array}{l}45.63 \\
(29.9569 .82)\end{array}$ & $\begin{array}{l}38.96 \\
(25.6958 .6)\end{array}$ & $\begin{array}{ll}1,442.11 & \\
(1,134.05 & 1,965.74)\end{array}$ & $\begin{array}{l}850.47 \\
(589.15 \\
1,306.45)\end{array}$ & $\begin{array}{l}591.64 \\
(403.56900 .02)\end{array}$ \\
\hline $\begin{array}{l}\text { Cardiovascular and circulatory } \\
\text { diseases }\end{array}$ & 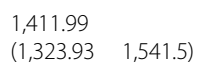 & 0.2 & 0.9 & 9.8 & 70.4 & 374.6 & 956.1 & $\begin{array}{l}621.16 \\
(583.59657 .16)\end{array}$ & $\begin{array}{l}790.83 \\
(714.8921 .4)\end{array}$ & $\begin{array}{ll}1,4937.05 & \\
(1,4229.38 & 1,6134.05)\end{array}$ & $\begin{array}{l}8,344.88 \\
(7,929.4 \quad 8,713.27)\end{array}$ & $\begin{array}{l}6,592.17 \\
(6,047.73 \quad 7,708.66)\end{array}$ \\
\hline Rheumatic heart disease & $\begin{array}{l}36.79 \\
(29.0345 .78)\end{array}$ & 0.0 & 0.0 & 0.1 & 1.3 & 9.4 & 25.7 & $\begin{array}{l}8.77 \\
(5.8311 .22)\end{array}$ & $\begin{array}{l}28.02 \\
(21.1136 .12)\end{array}$ & $\begin{array}{l}368.29 \\
(308.32428 .92)\end{array}$ & $\begin{array}{l}117.17 \\
(78.95138 .38)\end{array}$ & $\begin{array}{l}251.12 \\
(204.52302 .84)\end{array}$ \\
\hline
\end{tabular}


Table 2 Main causes of mortality and years of life lost (YLLs) by age groups and by gender, for the Spanish population in 2010 (Continued)

\begin{tabular}{|c|c|c|c|c|c|c|c|c|c|c|c|c|}
\hline Ischemic heart disease & $\begin{array}{l}626.56 \\
(577.22692 .18)\end{array}$ & 0.0 & 0.1 & 4.9 & 40.2 & 178.0 & 403.3 & $\begin{array}{l}308.45 \\
(282.37 \text { 349.41) }\end{array}$ & $\begin{array}{l}318.11 \\
(282.55370 .95)\end{array}$ & $\begin{array}{l}7,081 \\
(6,637.38 \quad 7,802.67)\end{array}$ & $\begin{array}{l}4,428.89 \\
(4,114.2 \quad 4,977.74)\end{array}$ & $\begin{array}{l}2,652.11 \\
(2,409.27 \quad 3,107.44)\end{array}$ \\
\hline Cerebrovascular disease & $\begin{array}{l}429.4 \\
(368.79561 .38)\end{array}$ & 0.0 & 0.1 & 1.9 & 14.0 & 103.2 & 310.0 & $\begin{array}{l}171.63 \\
(142.42222 .99)\end{array}$ & $\begin{array}{l}257.77 \\
(213.81366 .07)\end{array}$ & $\begin{array}{l}4,112.36 \\
(3,640.69 \quad 5,216.74)\end{array}$ & $\begin{array}{l}2,003.92 \\
(1,732.92 \quad 2,516.07)\end{array}$ & $\begin{array}{ll}2,108.44 & \\
(1,807.93 & 2,964.23)\end{array}$ \\
\hline Hypertensive heart disease & $\begin{array}{l}63.64 \\
(47.68 \text { 83.36) }\end{array}$ & 0.0 & 0.0 & 0.1 & 1.6 & 12.9 & 48.8 & $\begin{array}{l}21.25 \\
(15.4526 .68)\end{array}$ & $\begin{array}{l}42.38 \\
(27.8262 .1)\end{array}$ & $\begin{array}{l}566.48 \\
(445.62709 .6)\end{array}$ & $\begin{array}{l}242.98 \\
(183.82 \text { 285.61) }\end{array}$ & $\begin{array}{l}323.5 \\
(216.24 \quad 460.26)\end{array}$ \\
\hline $\begin{array}{l}\text { Cardiomyopathy and } \\
\text { myocarditis }\end{array}$ & $\begin{array}{l}50.88 \\
(43.6760 .5)\end{array}$ & 0.0 & 0.0 & 1.1 & 4.8 & 16.3 & 28.4 & $\begin{array}{l}28.21 \\
(22.5836 .59)\end{array}$ & $\begin{array}{l}22.67 \\
(18.1527 .22)\end{array}$ & $\begin{array}{l}697.3 \\
(611.01826 .99)\end{array}$ & $\begin{array}{l}470.92 \\
(389.76589 .69)\end{array}$ & $\begin{array}{l}226.37 \\
(192.37257 .76)\end{array}$ \\
\hline Atrial fibrillation and flutter & $\begin{array}{l}49.57 \\
(29.3278 .88)\end{array}$ & 0.0 & 0.0 & 0.0 & 0.3 & 8.8 & 40.5 & $\begin{array}{l}16.36 \\
(8.1730 .95)\end{array}$ & $\begin{array}{l}33.21 \\
(16.1560 .01)\end{array}$ & $\begin{array}{l}388.35 \\
(250.38588 .8)\end{array}$ & $\begin{array}{l}156.64 \\
(84.08278 .6)\end{array}$ & $\begin{array}{l}231.71 \\
(129.37389 .33)\end{array}$ \\
\hline Aortic aneurysm & $\begin{array}{l}22.1 \\
(16.23\end{array}$ & 0.0 & 0.0 & 0.1 & 1.8 & 9.8 & 10.2 & $\begin{array}{l}16.99 \\
(11.5323 .56)\end{array}$ & $\begin{array}{l}5.11 \\
(3.658 .83)\end{array}$ & $\begin{array}{l}308.17 \\
(224.6403 .06)\end{array}$ & $\begin{array}{l}251.95 \\
(169.97345 .83)\end{array}$ & $\begin{array}{l}56.22 \\
(45.1384 .66)\end{array}$ \\
\hline Peripheral vascular disease & $\begin{array}{l}13.51 \\
(7.8921 .83)\end{array}$ & 0.0 & 0.0 & 0.0 & 0.1 & 2.8 & 10.5 & $\begin{array}{l}4.27 \\
(1.778 .98)\end{array}$ & $\begin{array}{l}9.24 \\
(4.8416 .38)\end{array}$ & $\begin{array}{l}113.92 \\
(69.2182 .18)\end{array}$ & $\begin{array}{l}49.4 \\
(20.22100 .5)\end{array}$ & $\begin{array}{l}64.52 \\
(37.27 \\
(112.09)\end{array}$ \\
\hline Endocarditis & $\begin{array}{l}4.85 \\
(3.535 .85)\end{array}$ & 0.0 & 0.0 & 0.0 & 0.3 & 1.6 & 2.6 & $\begin{array}{l}2.32 \\
\left(\begin{array}{ll}1.68 & 2.94)\end{array}\right.\end{array}$ & $\begin{array}{l}2.53 \\
\left(\begin{array}{ll}1.48 & 3.34\end{array}\right)\end{array}$ & $\begin{array}{l}68.91 \\
(52.7180 .35)\end{array}$ & $\begin{array}{l}39.65 \\
(30.2547 .19)\end{array}$ & $\begin{array}{l}29.26 \\
(17.3236 .84)\end{array}$ \\
\hline $\begin{array}{l}\text { Other cardiovascular and } \\
\text { circulatory diseases }\end{array}$ & $\begin{array}{l}114.69 \\
(103.64128 .09)\end{array}$ & 0.0 & 0.0 & 1.1 & 5.2 & 31.8 & 76.3 & $\begin{array}{l}42.9 \\
(38.1647 .72)\end{array}$ & $\begin{array}{l}71.79 \\
(61.684 .61)\end{array}$ & $\begin{array}{l}1,232.26 \\
(1,151.54 \quad 1,317.41)\end{array}$ & $\begin{array}{l}583.36 \\
(538.22631 .15)\end{array}$ & $\begin{array}{l}648.9 \\
(582.6724 .27)\end{array}$ \\
\hline Chronic respiratory diseases & $\begin{array}{l}314.29 \\
(290.3340 .56)\end{array}$ & 0.0 & 0.0 & 0.9 & 7.7 & 85.1 & 220.4 & $\begin{array}{l}184.71 \\
(168.12 \text { 201.48) }\end{array}$ & $\begin{array}{l}129.58 \\
(112.04149 .2)\end{array}$ & $\begin{array}{l}3,042.65 \\
(2,862.02 \quad 3,232.05)\end{array}$ & $\begin{array}{l}1,988.91 \\
(1,853.32 \quad 2,124.11)\end{array}$ & $\begin{array}{ll}1,053.75 \\
(943.48 & 1,173.44)\end{array}$ \\
\hline $\begin{array}{l}\text { Chronic obstructive } \\
\text { pulmonary disease }\end{array}$ & $\begin{array}{l}186.69 \\
(161.55210 .12)\end{array}$ & 0.0 & 0.0 & 0.1 & 4.1 & 54.2 & 128.0 & $\begin{array}{l}127.89 \\
(102.9151 .05)\end{array}$ & 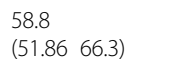 & $\begin{array}{ll}1,816.89 & \\
(1,581.96 & 2,035.79)\end{array}$ & $\begin{array}{l}1,350.53 \\
(1,113.46 \quad 1,566.59)\end{array}$ & $\begin{array}{l}466.36 \\
(424.85510 .99)\end{array}$ \\
\hline Pneumoconiosis & $\begin{array}{l}14.55 \\
(9.4921 .14)\end{array}$ & 0.0 & 0.0 & 0.0 & 0.3 & 4.1 & 10.1 & $\begin{array}{l}8.41 \\
(4.6813 .84)\end{array}$ & $\begin{array}{l}6.14 \\
(3.0810 .78)\end{array}$ & $\begin{array}{l}142.94 \\
(95.3207 .26)\end{array}$ & $\begin{array}{l}95.76 \\
(55.91154 .49)\end{array}$ & $\begin{array}{l}47.18 \\
(24.9778 .07)\end{array}$ \\
\hline Asthma & $\begin{array}{l}10.82 \\
(8.3114 .66)\end{array}$ & 0.0 & 0.0 & 0.0 & 0.5 & 3.0 & 7.0 & $\begin{array}{l}2.48 \\
(1.665 .71)\end{array}$ & $\begin{array}{l}8.34 \\
\left(\begin{array}{l}5.6 \\
10.39\end{array}\right)\end{array}$ & $\begin{array}{l}126.37 \\
(103.62173 .86)\end{array}$ & $\begin{array}{l}39.76 \\
(28.9283 .48)\end{array}$ & $\begin{array}{l}86.61 \\
(61.65101 .64)\end{array}$ \\
\hline $\begin{array}{l}\text { Interstitial lung disease and } \\
\text { pulmonary sarcoidosis }\end{array}$ & $\begin{array}{l}19.91 \\
(12.3229 .59)\end{array}$ & 0.0 & 0.0 & 0.0 & 1.0 & 8.9 & 10.0 & $\begin{array}{l}10.94 \\
(5.8119 .32)\end{array}$ & $\begin{array}{l}8.98 \\
(4.0415 .82)\end{array}$ & $\begin{array}{l}246.47 \\
(167.1353 .69)\end{array}$ & $\begin{array}{l}145.14 \\
(85.09237 .34)\end{array}$ & $\begin{array}{l}101.33 \\
(52.57165 .72)\end{array}$ \\
\hline $\begin{array}{l}\text { Other chronic respiratory } \\
\text { diseases }\end{array}$ & $\begin{array}{l}82.32 \\
(60.89108 .12)\end{array}$ & 0.0 & 0.0 & 0.2 & 1.6 & 14.9 & 65.3 & $\begin{array}{l}34.99 \\
(24.6253 .3)\end{array}$ & $\begin{array}{l}47.32 \\
(30.8169 .1)\end{array}$ & $\begin{array}{l}709.98 \\
(577.64969 .25)\end{array}$ & $\begin{array}{l}357.72 \\
(274.56541 .95)\end{array}$ & $\begin{array}{l}352.26 \\
(248.4502 .74)\end{array}$ \\
\hline Cirrhosis of the liver & $\begin{array}{l}88.53 \\
(77.98111 .17)\end{array}$ & 0.0 & 0.0 & 2.1 & 21.5 & 37.6 & 27.3 & $\begin{array}{l}54.89 \\
(46.8874 .57)\end{array}$ & $\begin{array}{l}33.64 \\
(27.9244 .82)\end{array}$ & $\begin{array}{l}1,735.13 \\
(1,547.01 \quad 2,184.28)\end{array}$ & $\begin{array}{l}1,222.34 \\
(1,061.28 \quad 1,660.84)\end{array}$ & $\begin{array}{l}512.79 \\
(438.15732 .49)\end{array}$ \\
\hline $\begin{array}{l}\text { Cirrhosis of the liver } \\
\text { secondary to hepatitis B }\end{array}$ & $\begin{array}{l}3.68 \\
(3.064 .61)\end{array}$ & 0.0 & 0.0 & 0.0 & 0.8 & 1.6 & 1.2 & $\begin{array}{l}1.93 \\
(1.52 .69)\end{array}$ & $\begin{array}{l}1.74 \\
\left(\begin{array}{ll}1.33 & 2.27\end{array}\right)\end{array}$ & $\begin{array}{l}66.56 \\
(55.7485 .12)\end{array}$ & $\begin{array}{l}40.9 \\
(31.6356 .41)\end{array}$ & $\begin{array}{l}25.66 \\
(19.8834 .09)\end{array}$ \\
\hline $\begin{array}{l}\text { Cirrhosis of the liver } \\
\text { secondary to hepatitis } C\end{array}$ & $\begin{array}{l}38.7 \\
(32.5549 .19)\end{array}$ & 0.0 & 0.0 & 0.4 & 7.2 & 17.5 & 13.6 & $\begin{array}{l}18.92 \\
(14.5926 .86)\end{array}$ & $\begin{array}{l}19.78 \\
(15.0326 .26)\end{array}$ & $\begin{array}{l}672.7 \\
(569.42857 .44)\end{array}$ & $\begin{array}{l}389.74 \\
(303.2550 .58)\end{array}$ & $\begin{array}{l}282.96 \\
(221.68386 .87)\end{array}$ \\
\hline $\begin{array}{l}\text { Cirrhosis of the liver } \\
\text { secondary to alcohol use }\end{array}$ & $\begin{array}{l}38.53 \\
(32.4150 .61)\end{array}$ & 0.0 & 0.0 & 1.1 & 10.9 & 15.9 & 10.6 & $\begin{array}{l}29.81 \\
(23.6941 .15)\end{array}$ & $\begin{array}{l}8.72 \\
(6.9311 .34)\end{array}$ & $\begin{array}{ll}812.93 & \\
(675.37 & 1,067.51)\end{array}$ & $\begin{array}{l}676.87 \\
(540.63931 .27)\end{array}$ & $\begin{array}{l}136.05 \\
(110.95182 .42)\end{array}$ \\
\hline Other cirrhosis of the liver & $\begin{array}{l}7.62 \\
(6.349 .58)\end{array}$ & 0.0 & 0.0 & 0.4 & 2.7 & 2.5 & 1.9 & $\begin{array}{l}4.24 \\
(3.296)\end{array}$ & $\begin{array}{l}3.39 \\
(2.634 .49)\end{array}$ & $\begin{array}{l}182.95 \\
(152.89235 .21)\end{array}$ & $\begin{array}{l}114.82 \\
(89.6162 .57)\end{array}$ & $\begin{array}{l}68.12 \\
(53.8496 .43)\end{array}$ \\
\hline $\begin{array}{l}\text { Digestive diseases } \\
\text { (except cirrhosis) }\end{array}$ & $\begin{array}{l}129.05 \\
(111.24 \quad 145.99)\end{array}$ & 0.0 & 0.0 & 0.9 & 6.1 & 35.4 & 86.5 & $\begin{array}{l}54.43 \\
(41.6865 .06)\end{array}$ & $\begin{array}{l}74.62 \\
(62.0187 .51)\end{array}$ & $\begin{array}{l}1,367.61 \\
(1,200.26 \quad 1,524.33)\end{array}$ & $\begin{array}{l}712.46 \\
(566.44835 .42)\end{array}$ & $\begin{array}{l}655.16 \\
(575.25735 .08)\end{array}$ \\
\hline Peptic ulcer disease & $\begin{array}{l}7.94 \\
(6.1212 .78)\end{array}$ & 0.0 & 0.0 & 0.0 & 0.5 & 2.3 & 5.0 & $\begin{array}{l}3.91 \\
(2.756 .09)\end{array}$ & $\begin{array}{l}4.03 \\
(2.787 .21)\end{array}$ & $\begin{array}{l}94.36 \\
(78.41133 .23)\end{array}$ & $\begin{array}{l}56.52 \\
(42.8976 .76)\end{array}$ & $\begin{array}{l}37.84 \\
(29.660 .57)\end{array}$ \\
\hline
\end{tabular}


Table 2 Main causes of mortality and years of life lost (YLLs) by age groups and by gender, for the Spanish population in 2010 (Continued)

\begin{tabular}{|c|c|c|c|c|c|c|c|c|c|c|c|c|}
\hline Gastritis and duodenitis & $\left.\begin{array}{ll}0.7 & \\
(0.38 & 1.34\end{array}\right)$ & 0.0 & 0.0 & 0.0 & 0.0 & 0.0 & 0.5 & $\begin{array}{ll}0.33 \\
(0.15 & 0.79)\end{array}$ & $\begin{array}{ll}0.37 \\
(0.17 & 0.83)\end{array}$ & $\begin{array}{l}7.71 \\
(4.6614)\end{array}$ & $\begin{array}{l}4.37 \\
(2.439 .64)\end{array}$ & $\begin{array}{l}3.34 \\
(1.856 .94)\end{array}$ \\
\hline Appendicitis & $\begin{array}{l}2.27 \\
\left(\begin{array}{ll}1.25 & 3.37\end{array}\right)\end{array}$ & 0.0 & 0.0 & 0.0 & 0.0 & 0.8 & 1.2 & $\begin{array}{l}0.99 \\
(0.391 .73)\end{array}$ & $\begin{array}{l}1.28 \\
\left(\begin{array}{ll}0.51 & 2.11)\end{array}\right.\end{array}$ & $\begin{array}{l}29.43 \\
(17.1140 .96)\end{array}$ & $\begin{array}{l}15.49 \\
(6.22 \text { 24.66) }\end{array}$ & $\begin{array}{l}13.94 \\
(5.8920 .06)\end{array}$ \\
\hline $\begin{array}{l}\text { Paralytic ileus and intestinal } \\
\text { obstruction without hernia }\end{array}$ & $\begin{array}{l}18.65 \\
(11.2227 .18)\end{array}$ & 0.0 & 0.0 & 0.0 & 0.3 & 4.2 & 13.9 & $\begin{array}{l}6.75 \\
(3.2410 .65)\end{array}$ & $\begin{array}{l}11.89 \\
(6.2719 .31)\end{array}$ & $\begin{array}{l}168.81 \\
(105.79226 .43)\end{array}$ & $\begin{array}{l}73.98 \\
(37.43 \text { 107.19) }\end{array}$ & $\begin{array}{l}94.82 \\
(52.72 \text { 141.84) }\end{array}$ \\
\hline Inguinal or femoral hernia & $\begin{array}{l}1.98 \\
\left(\begin{array}{ll}1.78 & 2.05)\end{array}\right.\end{array}$ & 0.0 & 0.0 & 0.0 & 0.0 & 0.4 & 1.4 & $\begin{array}{l}0.87 \\
\left(\begin{array}{ll}0.67 & 0.91)\end{array}\right.\end{array}$ & $\begin{array}{l}1.11 \\
(1.071 .16)\end{array}$ & $\begin{array}{l}19.83 \\
(17.6420 .41)\end{array}$ & $\begin{array}{l}10.61 \\
(8.4610 .99)\end{array}$ & $\begin{array}{l}9.22 \\
(99.53)\end{array}$ \\
\hline $\begin{array}{l}\text { Non-infective inflammatory } \\
\text { bowel disease }\end{array}$ & $\begin{array}{l}3.44 \\
(2.084 .92)\end{array}$ & 0.0 & 0.0 & 0.0 & 0.0 & 1.0 & 2.1 & $\begin{array}{l}1.38 \\
(0.772 .01)\end{array}$ & $\begin{array}{l}2.05 \\
(1.083 .31)\end{array}$ & $\begin{array}{l}42.73 \\
(27.9855 .05)\end{array}$ & $\begin{array}{l}21.53 \\
(12.8928 .54)\end{array}$ & $\begin{array}{l}21.2 \\
(12.3730 .96)\end{array}$ \\
\hline $\begin{array}{l}\text { Vascular disorders of } \\
\text { intestine }\end{array}$ & $\begin{array}{l}31.9 \\
(17.3957 .23)\end{array}$ & 0.0 & 0.0 & 0.0 & 0.9 & 8.9 & 21.9 & $\begin{array}{l}14.29 \\
(3.4238 .48)\end{array}$ & $\begin{array}{l}17.61 \\
(7.8328 .97)\end{array}$ & $\begin{array}{l}311.86 \\
(164.05601 .7)\end{array}$ & $\begin{array}{l}170.18 \\
(41.36457 .84)\end{array}$ & $\begin{array}{l}141.68 \\
(65.87231 .55)\end{array}$ \\
\hline $\begin{array}{l}\text { Gall bladder and bile duct } \\
\text { disease }\end{array}$ & $\begin{array}{l}17.51 \\
(12.1924 .68)\end{array}$ & 0.0 & 0.0 & 0.0 & 0.3 & 3.9 & 13.0 & $\begin{array}{l}6.69 \\
(3.9410 .06)\end{array}$ & $\begin{array}{l}10.82 \\
\left(\begin{array}{ll}6.51 & 17.59)\end{array}\right.\end{array}$ & $\begin{array}{l}162.17 \\
(122.46210 .64)\end{array}$ & $\begin{array}{l}74.29 \\
(46.15 \\
103.25)\end{array}$ & $\begin{array}{l}87.88 \\
(57.82 \\
129.7)\end{array}$ \\
\hline Pancreatitis & $\begin{array}{l}13.45 \\
(9.97 \quad 17.26)\end{array}$ & 0.0 & 0.0 & 0.2 & 1.5 & 4.7 & 7.0 & $\begin{array}{l}6.36 \\
(4.139 .22)\end{array}$ & $\begin{array}{l}7.08 \\
(4.7210 .08)\end{array}$ & $\begin{array}{l}190.96 \\
(146.04250 .94)\end{array}$ & $\begin{array}{l}113.47 \\
(77.21 \quad 169.87)\end{array}$ & $\begin{array}{l}77.5 \\
(54.84111 .18)\end{array}$ \\
\hline Other digestive diseases & $\begin{array}{l}31.22 \\
(21.139 .68)\end{array}$ & 0.0 & 0.0 & 0.1 & 1.6 & 8.9 & 20.4 & $\begin{array}{l}12.85 \\
(8.5816 .52)\end{array}$ & $\begin{array}{l}18.36 \\
(9.9526 .55)\end{array}$ & $\begin{array}{l}339.75 \\
(246.38418 .1)\end{array}$ & $\begin{array}{l}172.02 \\
(118.95215 .82)\end{array}$ & $\begin{array}{l}167.73 \\
(90.77234 .96)\end{array}$ \\
\hline Neurological disorders & $\begin{array}{l}298.8 \\
(200.34394 .65)\end{array}$ & 0.3 & 0.7 & 1.7 & 6.5 & 64.8 & 224.8 & $\begin{array}{l}109.69 \\
(77.39153 .19)\end{array}$ & $\begin{array}{l}189.11 \\
(95.13279 .64)\end{array}$ & $\begin{array}{l}2,744.5 \\
(2,092.35 \quad 3,423.72)\end{array}$ & $\begin{array}{ll}1,220.72 \\
(953.14 & 1,606.4)\end{array}$ & $\begin{array}{l}1,523.78 \\
(921.34 \quad 2,077)\end{array}$ \\
\hline $\begin{array}{l}\text { Alzheimer's disease and } \\
\text { other dementias }\end{array}$ & $\begin{array}{l}243.35 \\
(144.54342 .54)\end{array}$ & 0.0 & 0.0 & 0.0 & 1.0 & 44.2 & 198.0 & $\begin{array}{l}81.11 \\
(52.94126 .3)\end{array}$ & $\begin{array}{l}162.24 \\
(67.03254 .46)\end{array}$ & $\begin{array}{l}1,877.24 \\
(1,253.43 \quad 2,491)\end{array}$ & 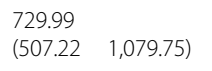 & $\begin{array}{ll}1,147.26 & \\
(548.76 & 1,699.76)\end{array}$ \\
\hline Parkinson's disease & $\begin{array}{l}29.53 \\
(18.6640 .27)\end{array}$ & 0.0 & 0.0 & 0.0 & 0.1 & 9.3 & 19.8 & $\begin{array}{l}14.86 \\
(7.6921 .9)\end{array}$ & $\begin{array}{l}14.67 \\
(7.22 \text { 22.47) }\end{array}$ & $\begin{array}{l}274.27 \\
(188.02366 .69)\end{array}$ & $\begin{array}{l}151.03 \\
(87.14227 .06)\end{array}$ & $\begin{array}{l}123.24 \\
\left(\begin{array}{ll}69.48 & 176.67)\end{array}\right.\end{array}$ \\
\hline Epilepsy & $\begin{array}{l}4.82 \\
(3.256 .35)\end{array}$ & 0.0 & 0.0 & 0.4 & 0.8 & 1.2 & 2.0 & $\begin{array}{l}2.47 \\
(1.6 \quad 3.31)\end{array}$ & $\begin{array}{l}2.36 \\
(1.253 .71)\end{array}$ & $\begin{array}{l}108.12 \\
(77.25127 .15)\end{array}$ & $\begin{array}{l}64.85 \\
(42.3382 .13)\end{array}$ & $\begin{array}{l}43.27 \\
(27.2255 .03)\end{array}$ \\
\hline Multiple sclerosis & $\begin{array}{l}1.95 \\
(1.422 .48)\end{array}$ & 0.0 & 0.0 & 0.0 & 0.8 & 0.7 & 0.2 & $\begin{array}{l}0.83 \\
(0.541 .11)\end{array}$ & $\begin{array}{l}1.12 \\
(0.7 \quad 1.57)\end{array}$ & $\begin{array}{l}54.54 \\
(39.8470)\end{array}$ & $\begin{array}{l}23.21 \\
(15.2631 .31)\end{array}$ & $\begin{array}{l}31.33 \\
(19.4944 .75)\end{array}$ \\
\hline Other neurological disorders & $\begin{array}{l}19.16 \\
(12.1426 .18)\end{array}$ & 0.3 & 0.3 & 0.9 & 3.5 & 9.2 & 4.7 & $\begin{array}{l}10.43 \\
(5.9515 .79)\end{array}$ & $\begin{array}{l}8.73 \\
(3.8214 .32)\end{array}$ & $\begin{array}{l}430.33 \\
(278.35593 .29)\end{array}$ & $\begin{array}{l}251.65 \\
(149.6392 .18)\end{array}$ & $\begin{array}{l}178.69 \\
(80.22282 .85)\end{array}$ \\
\hline $\begin{array}{l}\text { Mental and behavioral } \\
\text { disorders }\end{array}$ & $\begin{array}{l}13.49 \\
(9.7221 .5)\end{array}$ & 0.0 & 0.1 & 3.9 & 4.2 & 3.2 & 2.2 & $\begin{array}{l}10.37 \\
(7.1518 .63)\end{array}$ & $\begin{array}{l}3.13 \\
(1.834 .34)\end{array}$ & $\begin{array}{l}453.07 \\
(323.04797 .96)\end{array}$ & 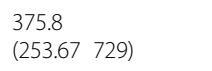 & $\begin{array}{l}77.26 \\
\left(\begin{array}{ll}48.94 & 109.57)\end{array}\right.\end{array}$ \\
\hline Schizophrenia & $\begin{array}{ll}1.17 & \\
(0.61 & 1.69)\end{array}$ & 0.0 & 0.0 & 0.0 & 0.0 & 0.4 & 0.4 & $\begin{array}{ll}0.61 & \\
(0.27 & 0.9)\end{array}$ & $\begin{array}{ll}0.56 & \\
(0.22 & 0.99)\end{array}$ & $\left.\begin{array}{ll}22.53 & \\
(12.28 & 29.9\end{array}\right)$ & $\begin{array}{l}13.51 \\
(6.0219 .24)\end{array}$ & $\begin{array}{l}9.02 \\
(3.7313 .98)\end{array}$ \\
\hline Alcohol use disorders & $\begin{array}{l}4.36 \\
(2.618 .97)\end{array}$ & 0.0 & 0.0 & 0.2 & 2.0 & 1.7 & 0.4 & $\begin{array}{l}3.82 \\
(2.058 .28)\end{array}$ & $\begin{array}{l}0.55 \\
(0.221 .13)\end{array}$ & $\begin{array}{l}124.99 \\
(77.51260 .19)\end{array}$ & $\begin{array}{l}109.43 \\
(61.15243 .7)\end{array}$ & $\begin{array}{l}15.57 \\
(6.0531 .99)\end{array}$ \\
\hline Drug use disorders & $\begin{array}{ll}6.98 \\
(3.87 & 12.75)\end{array}$ & 0.0 & 0.1 & 3.3 & 2.0 & 0.6 & 0.8 & $\begin{array}{l}5.52 \\
(2.6610 .82)\end{array}$ & $\begin{array}{l}1.47 \\
(0.542 .24)\end{array}$ & $\begin{array}{l}291.52 \\
(165.61588 .06)\end{array}$ & $\begin{array}{l}245.59 \\
(124.78541 .19)\end{array}$ & $\begin{array}{l}45.92 \\
(21.1272 .44)\end{array}$ \\
\hline Eating disorders & $\begin{array}{l}0.29 \\
\left(\begin{array}{ll}0.09 & 0.53)\end{array}\right.\end{array}$ & 0.0 & 0.0 & 0.0 & 0.0 & 0.0 & 0.2 & $\begin{array}{ll}0.1 & \\
(0.02 & 0.2)\end{array}$ & $\begin{array}{l}0.19 \\
(0.050 .39)\end{array}$ & $\begin{array}{l}4.53 \\
(2.046 .56)\end{array}$ & $\begin{array}{l}1.77 \\
\left(\begin{array}{ll}0.6 & 2.79\end{array}\right)\end{array}$ & $\begin{array}{l}2.76 \\
(1.074 .43)\end{array}$ \\
\hline $\begin{array}{l}\text { Other mental and behavioral } \\
\text { disorders }\end{array}$ & $\begin{array}{l}0.69 \\
\left(\begin{array}{ll}0.22 & 1.23\end{array}\right)\end{array}$ & 0.0 & 0.0 & 0.0 & 0.0 & 0.0 & 0.4 & 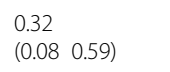 & $\begin{array}{l}0.37 \\
\left(\begin{array}{ll}0.08 & 0.77)\end{array}\right)\end{array}$ & $\begin{array}{l}9.5 \\
(3.9414 .28)\end{array}$ & $\begin{array}{l}5.5 \\
(1.798 .61)\end{array}$ & $\begin{array}{l}3.99 \\
(1.367 .01)\end{array}$ \\
\hline $\begin{array}{l}\text { Diabetes, urogenital, blood, and } \\
\text { endocrine diseases }\end{array}$ & $\begin{array}{l}280.14 \\
(242.63 \text { 353.84) }\end{array}$ & 0.3 & 0.9 & 2.2 & 11.2 & 79.6 & 185.6 & $\begin{array}{l}122.76 \\
(103.65167 .15)\end{array}$ & $\begin{array}{l}157.38 \\
(128.53205 .29)\end{array}$ & $\begin{array}{ll}3,032.98 \\
(2,645.05 & 4,078.86)\end{array}$ & $\begin{array}{ll}1,571.2 & \\
(1,318.85 & 2,311.61)\end{array}$ & $\begin{array}{l}1,461.78 \\
(1,239.48 \quad 1,988.42)\end{array}$ \\
\hline
\end{tabular}


Table 2 Main causes of mortality and years of life lost (YLLs) by age groups and by gender, for the Spanish population in 2010 (Continued)

\begin{tabular}{|c|c|c|c|c|c|c|c|c|c|c|c|c|}
\hline Diabetes mellitus & $\begin{array}{l}112.02 \\
(93.98151 .69)\end{array}$ & 0.0 & 0.0 & 0.2 & 3.9 & 34.7 & 73.3 & $\begin{array}{l}44.69 \\
(36.3164 .25)\end{array}$ & $\begin{array}{l}67.33 \\
(53.4795 .38)\end{array}$ & $\begin{array}{ll}1,146.26 \\
(995.43 & 1,592.83)\end{array}$ & $\begin{array}{l}561.77 \\
(479.06 \text { 867.39) }\end{array}$ & $\begin{array}{l}584.49 \\
(484.08 \text { 889.93) }\end{array}$ \\
\hline Acute glomerulonephritis & $\begin{array}{l}0.12 \\
(0.06 \quad 0.2)\end{array}$ & 0.0 & 0.0 & 0.0 & 0.0 & 0.0 & $<0.1$ & $\begin{array}{l}0.07 \\
(0.03 \quad 0.13)\end{array}$ & $\begin{array}{l}0.05 \\
\left(\begin{array}{ll}0.02 & 0.1\end{array}\right)\end{array}$ & $\begin{array}{l}2.5 \\
(1.133 .85)\end{array}$ & $\begin{array}{l}1.55 \\
\left(\begin{array}{ll}0.61 & 2.75)\end{array}\right.\end{array}$ & $\begin{array}{l}0.95 \\
\left(\begin{array}{ll}0.33 & 1.55)\end{array}\right)\end{array}$ \\
\hline Chronic kidney diseases & $\begin{array}{l}84.59 \\
(67.95110 .54)\end{array}$ & 0.0 & 0.0 & 0.2 & 2.4 & 21.6 & 60.0 & $\begin{array}{l}40.03 \\
(32.5456 .18)\end{array}$ & $\begin{array}{l}44.56 \\
(30.4267 .37)\end{array}$ & $\begin{array}{ll}819.87 & \\
(698.77 & 1,098.83)\end{array}$ & $\begin{array}{l}434.71 \\
(369.26 \text { 647.87) }\end{array}$ & $\begin{array}{l}385.15 \\
\text { (289.79 590.98) }\end{array}$ \\
\hline $\begin{array}{l}\text { Urinary diseases and male } \\
\text { infertility }\end{array}$ & $\begin{array}{l}44.35 \\
(26.8663 .32)\end{array}$ & 0.0 & 0.0 & 0.0 & 1.1 & 10.8 & 32.0 & $\begin{array}{l}21.01 \\
(10.933 .29)\end{array}$ & $\begin{array}{l}23.34 \\
(11.3538 .22)\end{array}$ & $\begin{array}{l}422.38 \\
(270.67566 .13)\end{array}$ & $\begin{array}{l}222.07 \\
(121.63335 .67)\end{array}$ & $\begin{array}{l}200.31 \\
(108.39294 .38)\end{array}$ \\
\hline Gynecological diseases & $\begin{array}{l}0.35 \\
(0.30 .39)\end{array}$ & 0.0 & 0.0 & 0.0 & 0.0 & 0.0 & 0.2 & * & $\begin{array}{l}0.35 \\
\left(\begin{array}{ll}0.3 & 0.39\end{array}\right)\end{array}$ & $\begin{array}{l}5.16 \\
(3.996 .54)\end{array}$ & * & $\begin{array}{l}5.16 \\
(3.996 .54)\end{array}$ \\
\hline $\begin{array}{l}\text { Hemoglobinopathies and } \\
\text { hemolytic anemia }\end{array}$ & $\begin{array}{l}5.12 \\
(3.777 .24)\end{array}$ & 0.0 & 0.0 & 0.0 & 0.0 & 1.3 & 3.3 & $\begin{array}{l}1.89 \\
\left(\begin{array}{ll}1.27 & 3.33\end{array}\right)\end{array}$ & $\begin{array}{l}3.23 \\
(2.154 .79)\end{array}$ & $\begin{array}{l}63.28 \\
(49.7989 .97)\end{array}$ & $\begin{array}{l}28.65 \\
(21.4847 .37)\end{array}$ & $\begin{array}{l}34.63 \\
(24.7552 .35)\end{array}$ \\
\hline $\begin{array}{l}\text { Other endocrine, nutritional, } \\
\text { blood, and immune } \\
\text { disorders }\end{array}$ & $\begin{array}{l}33.6 \\
(20.5784 .45)\end{array}$ & 0.3 & 0.8 & 1.3 & 3.4 & 11.0 & 16.8 & $\begin{array}{l}15.07 \\
(7.4350 .5)\end{array}$ & $\begin{array}{l}18.52 \\
(9.54 \quad 47.65)\end{array}$ & $\begin{array}{l}573.52 \\
(378.68\end{array}$ & $\begin{array}{l}322.44 \\
(178.48991 .18)\end{array}$ & $\begin{array}{l}251.08 \\
(145.49542 .18)\end{array}$ \\
\hline Musculoskeletal disorders & $\begin{array}{l}39.06 \\
(25.6466 .48)\end{array}$ & 0.0 & 0.0 & 0.3 & 1.5 & 7.9 & 29.3 & $\begin{array}{l}13.39 \\
(7.9229 .58)\end{array}$ & $\begin{array}{l}25.67 \\
(14.3648 .24)\end{array}$ & $\begin{array}{l}375.55 \\
(268.26 \text { 613.79) }\end{array}$ & $\begin{array}{l}143.94 \\
(96.43294 .08)\end{array}$ & $\begin{array}{l}231.6 \\
(141.74414 .1)\end{array}$ \\
\hline Rheumatoid arthritis & $\begin{array}{l}2.89 \\
(1.984 .19)\end{array}$ & 0.0 & 0.0 & 0.0 & 0.1 & 1.5 & 1.1 & 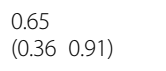 & $\begin{array}{l}2.24 \\
(1.423 .54)\end{array}$ & $\begin{array}{l}44.56 \\
(31.5768 .08)\end{array}$ & $\begin{array}{l}11.65 \\
(6.7915 .99)\end{array}$ & $\begin{array}{l}32.91 \\
(2257.69)\end{array}$ \\
\hline $\begin{array}{l}\text { Other musculoskeletal } \\
\text { disorders }\end{array}$ & $\begin{array}{l}36.17 \\
(23.9362 .55)\end{array}$ & 0.0 & 0.0 & 0.2 & 1.3 & 6.5 & 28.2 & $\begin{array}{l}12.74 \\
(7.3228 .65)\end{array}$ & $\begin{array}{l}23.43 \\
(13.0742 .38)\end{array}$ & $\begin{array}{l}330.99 \\
(241.09564 .59)\end{array}$ & $\begin{array}{l}132.3 \\
(86.48278 .21)\end{array}$ & $\begin{array}{l}198.69 \\
(129.47310 .74)\end{array}$ \\
\hline $\begin{array}{l}\text { Other non-communicable } \\
\text { diseases }\end{array}$ & $\begin{array}{l}26.82 \\
(21.1232 .38)\end{array}$ & 4.9 & 1.3 & 1.1 & 1.7 & 4.1 & 13.7 & $\begin{array}{l}10.76 \\
(8.6813 .33)\end{array}$ & $\begin{array}{l}16.06 \\
(11.3221 .15)\end{array}$ & $\begin{array}{l}793.69 \\
(700.07998 .5)\end{array}$ & $\begin{array}{l}408.18 \\
(349.11554 .42)\end{array}$ & $\begin{array}{l}385.51 \\
(323.19522 .23)\end{array}$ \\
\hline Congenital anomalies & $\begin{array}{l}15.23 \\
(12.8918 .32)\end{array}$ & 4.2 & 1.3 & 1.1 & 1.5 & 2.0 & 5.3 & $\begin{array}{l}6.84 \\
(5.668 .92)\end{array}$ & $\begin{array}{l}8.38 \\
(6.4110 .77)\end{array}$ & $\begin{array}{l}637.65 \\
(561.04 \text { 826.64) }\end{array}$ & $\begin{array}{l}336.37 \\
(283.44 \text { 482.44) }\end{array}$ & $\begin{array}{l}301.28 \\
(254.05437 .58)\end{array}$ \\
\hline $\begin{array}{l}\text { Skin and subcutaneous } \\
\text { diseases }\end{array}$ & $\begin{array}{l}10.88 \\
(6.3615 .66)\end{array}$ & 0.0 & 0.0 & 0.0 & 0.0 & 2.2 & 8.4 & $\begin{array}{l}3.53 \\
(1.835 .17)\end{array}$ & $\begin{array}{l}7.36 \\
(3.2611 .69)\end{array}$ & $\begin{array}{l}94.99 \\
(60.79124 .7)\end{array}$ & $\begin{array}{l}37.8 \\
(22.5150 .55)\end{array}$ & $\begin{array}{l}57.19 \\
(28.4583 .86)\end{array}$ \\
\hline $\begin{array}{l}\text { Sudden infant death } \\
\text { syndrome }\end{array}$ & $\begin{array}{l}0.71 \\
\left(\begin{array}{ll}0.37 & 1.3)\end{array}\right.\end{array}$ & 0.7 & 0.0 & 0.0 & 0.0 & 0.0 & 0.0 & $\begin{array}{ll}0.4 & \\
(0.16 & 0.89)\end{array}$ & $\begin{array}{ll}0.31 & \\
(0.12 & 0.74)\end{array}$ & $\begin{array}{l}61.05 \\
(31.43111 .36)\end{array}$ & $\begin{array}{l}34 \\
(14.13 \\
76.52)\end{array}$ & $\begin{array}{l}27.04 \\
(1063.55)\end{array}$ \\
\hline Injuries & $\begin{array}{l}170.04 \\
(148.35187 .71)\end{array}$ & 0.3 & 6.1 & 29.5 & 34.1 & 41.7 & 58.4 & $\begin{array}{l}106.22 \\
(90.64120 .45)\end{array}$ & $\begin{array}{l}63.81 \\
(51.3976 .28)\end{array}$ & $\begin{array}{ll}4,467.58 & \\
(3,963.64 & 4,986.31)\end{array}$ & $\begin{array}{l}3,292.76 \\
(2,796.78 \quad 3,744.15)\end{array}$ & $\begin{array}{ll}1,174.82 & \\
(1,034.86 & 1,351.47)\end{array}$ \\
\hline Transport injuries & $\begin{array}{l}43.13 \\
(37.1758 .97)\end{array}$ & 0.1 & 3.6 & 12.6 & 11.0 & 10.4 & 5.4 & $\begin{array}{l}31.86 \\
(25.7245 .25)\end{array}$ & $\begin{array}{l}11.27 \\
(9.316 .49)\end{array}$ & $\begin{array}{l}1,619.03 \\
(1,392.59 \quad 2,120.51)\end{array}$ & $\begin{array}{l}1,251.7 \\
(1,009.35 \quad 1,695.55)\end{array}$ & $\begin{array}{l}367.33 \\
(311.6513 .85)\end{array}$ \\
\hline Road injury & $\left.\begin{array}{ll}39.5 & \\
(34.03 & 54.39\end{array}\right)$ & 0.0 & 3.5 & 11.9 & 9.9 & 9.3 & 4.9 & $\begin{array}{l}28.91 \\
(23.741 .92)\end{array}$ & $\begin{array}{l}10.59 \\
(8.916 .96)\end{array}$ & $\begin{array}{ll}1,495.72 & \\
(1,292.76 & 1,960.84)\end{array}$ & $\begin{array}{l}1,151.98 \\
(940.51 \quad 1,613.62)\end{array}$ & $\begin{array}{l}343.74 \\
(298.04519 .39)\end{array}$ \\
\hline Other transport injury & $\begin{array}{l}3.63 \\
(2.554 .38)\end{array}$ & 0.0 & 0.1 & 0.9 & 1.1 & 1.2 & 0.5 & $\begin{array}{l}2.95 \\
\left(\begin{array}{ll}1.89 & 3.64)\end{array}\right.\end{array}$ & $\begin{array}{l}0.68 \\
(0.410 .94)\end{array}$ & $\begin{array}{l}123.31 \\
(84.2148 .87)\end{array}$ & $\begin{array}{l}99.72 \\
\left(\begin{array}{ll}65 & 123.8\end{array}\right)\end{array}$ & $\begin{array}{l}23.59 \\
(13.1931 .95)\end{array}$ \\
\hline $\begin{array}{l}\text { Unintentional injuries other } \\
\text { than transport injuries }\end{array}$ & $\begin{array}{l}86.75 \\
(66.99104 .08)\end{array}$ & 0.2 & 1.5 & 7.1 & 10.1 & 20.6 & 47.2 & $\begin{array}{l}44.43 \\
(34.2553 .05)\end{array}$ & $\begin{array}{l}42.32 \\
(29.0355 .39)\end{array}$ & $\begin{array}{ll}1,528.68 & \\
(1,254.66 & 1,728.1)\end{array}$ & $\begin{array}{l}1,042.66 \\
(815 \quad 1,229.43)\end{array}$ & $\begin{array}{l}486.02 \\
(376.59575 .11)\end{array}$ \\
\hline Falls & $\begin{array}{l}30.18 \\
(18.17 \text { 39.84) }\end{array}$ & 0.0 & 0.1 & 1.5 & 2.9 & 7.5 & 18.0 & $\begin{array}{l}14.31 \\
(7.5119 .64)\end{array}$ & $\begin{array}{l}15.87 \\
(8.0223 .56)\end{array}$ & $\begin{array}{l}447.58 \\
(283.13557 .46)\end{array}$ & $\begin{array}{l}290.71 \\
(160.9367 .9)\end{array}$ & $\begin{array}{l}156.87 \\
(86.49210 .64)\end{array}$ \\
\hline Drowning & $\begin{array}{l}6 \\
(4.887 .84)\end{array}$ & 0.0 & 0.5 & 1.3 & 1.6 & 1.8 & 0.8 & $\begin{array}{l}4.75 \\
(3.716 .73)\end{array}$ & $\left.\begin{array}{ll}1.24 & \\
(0.93 & 1.72\end{array}\right)$ & $\begin{array}{l}211.72 \\
(171.89283 .97)\end{array}$ & $\begin{array}{l}174.93 \\
(136.46247 .02)\end{array}$ & $\begin{array}{l}36.79 \\
(28.1550 .44)\end{array}$ \\
\hline $\begin{array}{l}\text { Fire, heat, and hot } \\
\text { substances }\end{array}$ & $\begin{array}{l}3.68 \\
(2.885 .15)\end{array}$ & 0.0 & 0.0 & 0.1 & 0.4 & 0.9 & 1.9 & $\begin{array}{l}1.91 \\
\left(\begin{array}{ll}1.41 & 2.8\end{array}\right)\end{array}$ & $\begin{array}{l}1.78 \\
\left(\begin{array}{ll}1.2 & 2.94\end{array}\right)\end{array}$ & $\begin{array}{l}70.8 \\
(57.8997 .19)\end{array}$ & $\begin{array}{ll}45.6 \\
(33.42 & 69.01)\end{array}$ & $\begin{array}{ll}25.2 & \\
(18.93 & 38.16)\end{array}$ \\
\hline
\end{tabular}


Table 2 Main causes of mortality and years of life lost (YLLs) by age groups and by gender, for the Spanish population in 2010 (Continued)

\begin{tabular}{|c|c|c|c|c|c|c|c|c|c|c|c|c|}
\hline Poisoning & $\begin{array}{ll}4.4 \\
(2.11 & 6.11)\end{array}$ & 0.0 & 0.0 & 1.6 & 1.2 & 0.6 & 0.8 & $\begin{array}{ll}2.98 \\
(1.074 .44)\end{array}$ & $\left.\begin{array}{ll}1.42 & \\
(0.61 & 1.99\end{array}\right)$ & $\begin{array}{l}158.83 \\
(71.04225 .89)\end{array}$ & $\begin{array}{l}120.96 \\
(40.98 \quad 185.67)\end{array}$ & $\begin{array}{l}37.88 \\
(18.8349 .55)\end{array}$ \\
\hline $\begin{array}{l}\text { Exposure to mechanical } \\
\text { forces }\end{array}$ & $\begin{array}{l}3.13 \\
(1.834 .37)\end{array}$ & 0.0 & 0.0 & 0.7 & 1.1 & 0.7 & 0.4 & $\begin{array}{l}2.59 \\
\left(\begin{array}{ll}1.37 & 3.91\end{array}\right)\end{array}$ & $\begin{array}{l}0.54 \\
(0.210 .84)\end{array}$ & $\begin{array}{l}110.16 \\
(64.39170 .53)\end{array}$ & $\begin{array}{l}97.15 \\
(52.91 \text { 157.16) }\end{array}$ & $\begin{array}{l}13.01 \\
\left(\begin{array}{ll}6.317 .41)\end{array}\right.\end{array}$ \\
\hline $\begin{array}{l}\text { Adverse effects of medical } \\
\text { treatment }\end{array}$ & $\begin{array}{l}9.25 \\
(5.8914 .86)\end{array}$ & 0.0 & 0.0 & 0.0 & 0.8 & 3.0 & 5.2 & $\begin{array}{l}3.72 \\
(2.854 .92)\end{array}$ & $\begin{array}{l}5.53 \\
(2.3811 .18)\end{array}$ & $\begin{array}{l}124.7 \\
(92.38 \text { 189.32) }\end{array}$ & $\begin{array}{l}61.82 \\
(50.9375 .98)\end{array}$ & $\begin{array}{l}62.88 \\
(32.01124 .6)\end{array}$ \\
\hline Animal contact & $\begin{array}{l}0.23 \\
(0.160 .33)\end{array}$ & 0.0 & 0.0 & 0.0 & 0.0 & 0.0 & $<0.1$ & $\begin{array}{l}0.17 \\
\left(\begin{array}{ll}0.1 & 0.25\end{array}\right)\end{array}$ & $\begin{array}{l}0.06 \\
(0.030 .1)\end{array}$ & $\begin{array}{l}6.95 \\
\left(\begin{array}{ll}4.67 & 10.13\end{array}\right)\end{array}$ & $\begin{array}{l}5.57 \\
(3.38 .48)\end{array}$ & $\begin{array}{l}1.38 \\
(0.822 .26)\end{array}$ \\
\hline $\begin{array}{l}\text { Unintentional injuries not } \\
\text { classified elsewhere }\end{array}$ & $\begin{array}{l}29.87 \\
(20.7240 .43)\end{array}$ & 0.1 & 0.1 & 1.4 & 2.2 & 6.0 & 20.1 & $\begin{array}{l}14 \\
(10.17 \text { 19.39) }\end{array}$ & $\begin{array}{l}15.87 \\
(7.8825 .14)\end{array}$ & $\begin{array}{l}397.93 \\
(328.14565 .3)\end{array}$ & $\begin{array}{l}245.92 \\
(194.62407 .48)\end{array}$ & $\begin{array}{l}152.01 \\
(106.27205 .69)\end{array}$ \\
\hline $\begin{array}{l}\text { Self-harm and interpersonal } \\
\text { violence }\end{array}$ & $\begin{array}{l}40.16 \\
(29.9251 .78)\end{array}$ & 0.0 & 0.6 & 9.9 & 12.9 & 10.7 & 5.8 & $\begin{array}{l}29.94 \\
(20.8140 .68)\end{array}$ & $\begin{array}{l}10.22 \\
(7.1114 .48)\end{array}$ & $\begin{array}{l}1,319.87 \\
(925.79 \\
1,566.26)\end{array}$ & $\begin{array}{l}998.4 \\
(662.08 \\
1251.41)\end{array}$ & $\begin{array}{l}321.47 \\
(206.88412 .94)\end{array}$ \\
\hline Self-harm & $\begin{array}{l}35.73 \\
(25.8447 .01)\end{array}$ & 0.0 & 0.5 & 8.2 & 11.5 & 10.0 & 5.4 & $\begin{array}{l}26.91 \\
(17.6737 .57)\end{array}$ & $\begin{array}{l}8.82 \\
(5.9513 .1)\end{array}$ & $\begin{array}{l}1,139.19 \\
(743.97 \quad 1,364.03)\end{array}$ & $\begin{array}{l}870.51 \\
(539.56 \\
1,112.22)\end{array}$ & $\begin{array}{l}268.69 \\
(162.15360 .64)\end{array}$ \\
\hline Interpersonal violence & $\begin{array}{l}4.43 \\
(3.286 .27)\end{array}$ & 0.0 & 0.1 & 1.7 & 1.4 & 0.7 & 0.4 & $\begin{array}{l}3.03 \\
(1.994 .87)\end{array}$ & $\begin{array}{l}1.4 \\
\left(\begin{array}{ll}1 & 1.69\end{array}\right)\end{array}$ & $\begin{array}{l}180.68 \\
(131.92 \quad 255.73)\end{array}$ & $\begin{array}{l}127.9 \\
(81.49197 .53)\end{array}$ & $\begin{array}{l}52.78 \\
(36.9463 .05)\end{array}$ \\
\hline
\end{tabular}

Data are presented as mean values and uncertainty intervals (Lower, Upper).

Mortality s uncertainty intervals in the six age groups (original age groups are 20) are not included in the table and will be given upon request. * not applicable. 
diabetes, urogenital, blood, and endocrine diseases (7.8\%) (Figure 1).

As shown in Table 2, 86\% of total YLLs were due to non-communicable diseases, with injuries and communicable diseases accounting for $8 \%$ and $6 \%$, respectively (see specific ranking in Figure 2). In 2010, regardless of gender, the leading specific cause for YLLs was neoplasms followed by cardiovascular and circulatory diseases.

In the top twenty causes of YLLs, the ranking (\% of change between the number of YLLs) changed between 1990 and 2010 (Figure 2). Remarkably, due to a large decrease in the number of YLLs, cardiovascular and circulatory diseases dropped from the first cause in 1990 to the second cause of YLLs in 2010 (decreased by 18\%). Neoplasms were the leading cause in 2010 (the second in 1990), and increased almost $12 \%$ in 2010 compared to 1990. Transport injuries were the seventh cause (third in 1990), and the chronic respiratory diseases were the third cause (fourth in 1990). Unintentional injuries other than transport injuries were the eighth cause (fifth in 1990), and decreased by approximately 46\% since 1990 . Diabetes, urogenital, blood, and endocrine diseases were the fourth cause (sixth in 1990), increasing by almost $10 \%$. The burden of YLLs in 2010, attributable to cirrhosis of the liver as well as diarrhea, lower respiratory infections, meningitis, and other common infectious diseases, decreased compared with 1990 (seventh to sixth cause and eight to ninth position, respectively). The percentage of YLLs attributable to self-harm and interpersonal violence, neonatal disorders, other noncommunicable diseases, and HIV/AIDS and tuberculosis, decreased by almost 9\%, 32\%, 35\%, and 55\% between 1990 and 2010, respectively. In contrast, YLLs attributable to neurological disorders increased by $134 \%$ and were the fifth leading cause in 2010, while YLLs due to mental and behavioral disorders increased by $29 \%$ between 1990 and 2010 (Figure 2).

Table 3 presents all causes of DALYs and YLDs in the Spanish population between 1990 and 2010. In this 20year period, there was an increase in the overall burden of YLDs of almost 26\%. In the broader classification terms (between 1990 and 2010), musculoskeletal disorders, mental and behavioral disorders, and diabetes, urogenital, blood, and endocrine diseases were the three main contributors to the years lived with disability in Spain. Focusing only in 2010, the largest contributors to the burden of YLDs, were the musculoskeletal disorders (26\%) and the mental and behavioral disorders (22\%). The group of diabetes, urogenital, blood, and endocrine disorders had an important contribution to the burden of disability (10\%), followed by the group of other noncommunicable disorders (9\%), neurological disorders (8\%), and other unintentional injuries except for transportation injuries $(7 \%)$.

Putting premature mortality and disability together in terms of DALYs provides an overall picture of the leading health problems in Spain. Between 1990 and 2010, there was a slight increase (5.4\%) in DALYs (i.e., overall health burden) in the overall Spanish population (from $104,615.9$ in 1990 to $110,269.4$ in 2010). The dominant causes of DALYs in 2010 were neoplasms, cardiovascular and circulatory diseases, musculoskeletal disorders, mental and behavioral disorders, and diabetes, urogenital, blood, and endocrine diseases, which accounted for nearly $59 \%$ of all DALYs (Table 3).

The top five leading causes for DALYs remained similar in both 1990 and 2010, despite the decreases or increases in the number of DALYs for each cause (\% of change between the numbers of DALYs). There was only

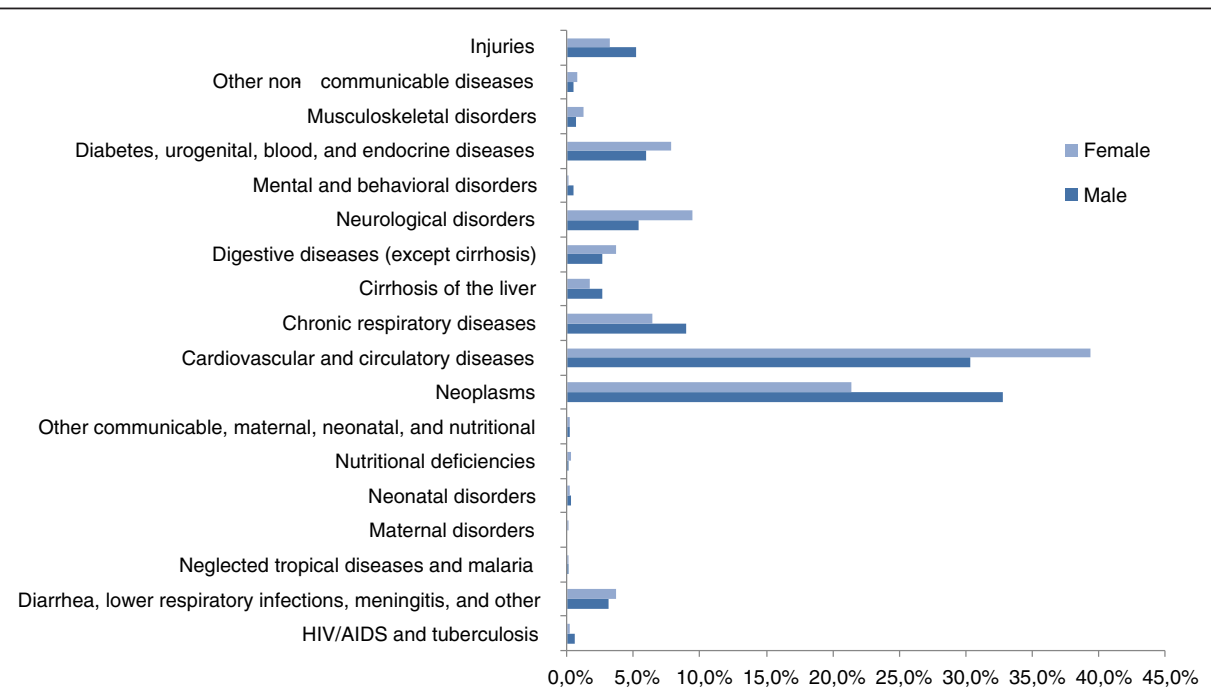

Figure 1 Spanish deaths in 2010 for males (204,990 total deaths) and females (200,750 total deaths) at all ages. 


\begin{tabular}{|c|c|c|c|c|}
\hline \multicolumn{2}{|r|}{1990 Main causes for YLLS } & \multicolumn{2}{|r|}{2010 Main causes for YLLs } & \multirow{2}{*}{$\begin{aligned} \% \Delta \\
12 \%\end{aligned}$} \\
\hline 1 & Cardiovascular and circulatory diseases & 1 & Neoplasms & \\
\hline 2 & Neoplasms & 2 & Cardiovascular and circulatory diseases & $-18 \%$ \\
\hline 3 & Transport injuries & 3 & Chronic respiratory diseases & $-17 \%$ \\
\hline 4 & Chronic respiratory diseases & 4 & Diabetes, urogenital, blood, and endocrine diseases & $10 \%$ \\
\hline 5 & Unintentional injuries other than transport injuries & 5 & Neurological disorders & $134 \%$ \\
\hline 6 & Diabetes, urogenital, blood, and endocrine diseases & 6 & Cirrhosis of the liver & $-25 \%$ \\
\hline 7 & Cirrhosis of the liver & 7 & Transport injuries & $-57 \%$ \\
\hline \multirow{2}{*}{8} & \multirow{3}{*}{$\begin{array}{l}\text { Diarrhea, lower respiratory infections, meningitis, and other } \\
\text { common infectious diseases } \\
\text { Self-harm and interpersonal violence }\end{array}$} & 8 & Unintentional injuries other than transport injuries & $-46 \%$ \\
\hline & & \multirow[t]{2}{*}{9} & \multirow{2}{*}{$\begin{array}{l}\text { Diarrhea, lower respiratory infections, meningitis, and other } \\
\text { common infectious diseases }\end{array}$} & \multirow[t]{2}{*}{$-16 \%$} \\
\hline 9 & & & & \\
\hline 10 & Othernon-communicable diseases & 10 & Digestive diseases (except cirrhosis) & $9 \%$ \\
\hline 11 & Digestive diseases (except cirrhosis) & 11 & Self-harm and interpersonal violence & $-9 \%$ \\
\hline 12 & HIV/AIDS and tuberculosis & 12 & Neonataldisorders & $-32 \%$ \\
\hline 13 & Neonatal disorders & 13 & Othernon-communicable diseases & $-39 \%$ \\
\hline 14 & Neurological disorders & 14 & HIV/AIDS and tuberculosis & $-55 \%$ \\
\hline 15 & Mental and behavioral disorders & 15 & Mental and behavioral disorders & $29 \%$ \\
\hline 16 & Musculoskeletaldisorders & 16 & Musculoskeletal disorders & $51 \%$ \\
\hline 17 & Other communicable, maternal, neonatal, and nutritional disorders & 17 & Other communicable, maternal, neonatal, and nutritional disorders & $13 \%$ \\
\hline 18 & Nutritional deficiencies & 18 & Nutritional deficiencies & $4 \%$ \\
\hline 19 & Neglected tropical diseases and malaria & 19 & Neglected tropical diseases and malaria & $-19 \%$ \\
\hline 20 & Maternal disorders & 20 & Maternal disorders & $-29 \%$ \\
\hline
\end{tabular}

a slight change in numerical order between the first two causes. Due to a large decrease in the number of DALYs (11\%), cardiovascular and circulatory diseases dropped to the second cause of DALYs in 2010 and neoplasms reached the first position increasing by 14\% from 1990 . Between the sixth and the eleventh positions the causes for DALYs changed only slightly. The transport injuries dropped from the ninth position in 1990s to the tenth in 2010, due to a large decrease (38\%) in the number of DALYs. The neurological disorders, with a $61 \%$ increase in DALYs, achieved the sixth position in 2010 from its tenth position in 1990. The HIV/AIDS and tuberculosis cause dropped one position and neonatal disorders increased one position in the list of causes for DALYs for the year 2010. Finally, the last four causes among the 20 top ranking of DALYs in Spain remained completely stable between 1990 and 2010, despite the changes in disability (Figure 3).

Summarizing the above data, in 2010, the leading causes for DALYs among newborn children ( $\left.\begin{array}{ll}0 & 1 \\ \text { years old }\end{array}\right)$ were neonatal disorders and the other communicable diseases group. The leading causes for DALYs in the younger group (5 to 44 years old) in the Spanish population were mental and behavioral disorders and musculoskeletal disorders, while in the middle aged and older adults groups the leading causes shifted to cardiovascular and circulatory diseases and neoplasms (Figure 4).

In both 1990 and 2010, there was a high consistency across European countries regarding the top causes of
YLDs being major depressive disorders, musculoskeletal disorders, low back and neck pain, and diabetes, as well as injuries (i.e., falls). YLDs caused by asthma, anxiety disorders, and chronic obstructive pulmonary disease ranked lower in Spain compared with other southern European countries, in both 1990 and 2010 (Figure 5).

From 1990 to 2010 overall DALYs attributable to noncommunicable diseases in Spain increased by 897,900 from $8,626,000$ to $9,523,900$. When standardizing the DALYs of 2010 to the 1990 population, the number of DALYs which would have increased was estimated to be $1,250,900$. Thus, the actual population health improved since the demographic changes would have caused a higher increase in DALYs than the actual 2010 DALYs. Changes in DALYs mostly correspond to population increase and are secondary to population ageing. Moreover, the highest difference was attributed to those 40 years and older.

\section{Discussion}

During the past 20 years, substantial changes have taken place in the relative impact of burden of diseases in Spain: while musculoskeletal disorders have increased by $28 \%$ (rank 3 as major cause of DALYs), mental and behavioral disorders have increased by $23 \%$ (rank 4), neurological disorders by 61\% (rank 6), and neoplasms by $14 \%$ (rank 1). On the other hand, cardiovascular and circulatory diseases have decreased by $11 \%$ (now rank 2), transport injuries by $38 \%$ (rank 10), and chronic 
Table 3 Comparison of the main causes of disability-adjusted life years (DALYs) and years lived with disability (YLDs), in 1990 and 2010 , for the Spanish population

\begin{tabular}{|c|c|c|c|c|c|c|}
\hline \multicolumn{4}{|l|}{ YLDs (hundreds) } & \multicolumn{3}{|l|}{ DALYs (hundreds) } \\
\hline & 1990 & 2010 & $\% \Delta$ & 1990 & 2010 & $\% \Delta$ \\
\hline All causes & $44,316.8$ & $55,706.6$ & $25.7 \%$ & $104,615.9$ & $110,269.4$ & $5.4 \%$ \\
\hline $\begin{array}{l}\text { Communicable, maternal, neonatal, and } \\
\text { nutritional disorders }\end{array}$ & $1,914.7(1,415.32,628.9)$ & $1,942.5(1,377.82,767.8)$ & $1 \%$ & $6,394.8(5,773.67,126)$ & $5,067.4(4,450.85,848.2)$ & $-21 \%$ \\
\hline HIV/AIDS and tuberculosis & $285.4(202.5384 .5)$ & $183(128.5248 .3)$ & $-36 \%$ & $1,532.1(1,356.21,720.5)$ & $738.3(643.6844 .3)$ & $-52 \%$ \\
\hline Tuberculosis & $114.8(71.7$ 171.8) & $84.4(50.8129 .2)$ & $-26 \%$ & 340.6 (274 409.3) & $175.9(136.8230 .7)$ & $-48 \%$ \\
\hline HIV/AIDS & 170.6 (123.2 223.1) & $98.6(70.8131 .4)$ & $-42 \%$ & $1,191.6(1,042 \quad 1,360.4)$ & $562.4(478.8651 .9)$ & $-53 \%$ \\
\hline HIV disease resulting in mycobacterial infection & $12.1\left(\begin{array}{ll}6.7 & 19.4\end{array}\right)$ & $4.7\left(\begin{array}{ll}2 & 8.3\end{array}\right)$ & $-62 \%$ & $59.8(51.569 .1)$ & $19.6(1624.1)$ & $-67 \%$ \\
\hline $\begin{array}{l}\text { HIV disease resulting in other specified or } \\
\text { unspecified diseases }\end{array}$ & 158.4 (114.2 206.7) & $93.9(67.9$ 125.4) & $-41 \%$ & 1,131.7 (993.6 1,271.3) & $542.8(469.8$ 629.4) & $-52 \%$ \\
\hline $\begin{array}{l}\text { Diarrhea, lower respiratory infections, meningitis, and } \\
\text { other common infectious diseases }\end{array}$ & $858.5(590.7$ 1249) & 925.1 (621.7 1339.5) & $8 \%$ & $2,555.5(2,206.4 \quad 3,004.8)$ & 2,358.1 (1,992.4 2,814.4) & $-8 \%$ \\
\hline Diarrheal diseases & $306.2(201.3440 .4)$ & $363.2(238.6520 .2)$ & $19 \%$ & 358.6 (252.5 493.1) & 428.9 (305.6 585.7) & $20 \%$ \\
\hline Typhoid and paratyphoid fevers & $0\left(\begin{array}{ll}0 & 0.1\end{array}\right)$ & $0\left(\begin{array}{ll}0 & 0.1\end{array}\right)$ & $27 \%$ & $1.1(0.12)$ & $1\left(\begin{array}{ll}0.1 & 2\end{array}\right)$ & $-4 \%$ \\
\hline Lower respiratory infections & $44.3(29.7 \quad 62)$ & $48.4(3366.8)$ & $9 \%$ & $1391.6(1227.51612 .5)$ & $1,275.9(1,096 \quad 1,523.9)$ & $-8 \%$ \\
\hline Upper respiratory infections & 219.3 (106.2 390.9) & 227.8 (110.9 429.7) & $4 \%$ & $221\left(\begin{array}{lll}107.8 & 392.7\end{array}\right)$ & 228.4 (111.5 430.3) & $3 \%$ \\
\hline Otitis media & 211.6 (127.1 339.9) & $211.9\left(\begin{array}{lll}120.7 & 361\end{array}\right)$ & $0.2 \%$ & $213.6(127.9341 .7)$ & $213.3(123 \quad 363.3)$ & $0 \%$ \\
\hline Meningitis & $52.1(33.7$ 77.9) & $46.8(2971.8)$ & $-10 \%$ & $261.9(229.8309)$ & 153.7 (129.1 183.3) & $-41 \%$ \\
\hline Pneumococcal meningitis & $10.2\left(\begin{array}{ll}6.2 & 15.4\end{array}\right)$ & $6.7(3.810 .6)$ & $-34 \%$ & $39.8(33.247 .3)$ & $21.8(17.626 .7)$ & $-45 \%$ \\
\hline $\mathrm{H}$ influenza type $\mathrm{B}$ meningitis & $7.4(4.311 .8)$ & $3.1(1.65 .1)$ & $-58 \%$ & $33.7(27.242 .1)$ & $13.6(10.817 .3)$ & $-60 \%$ \\
\hline Meningococcal infection & $16.6(10.225 .7)$ & $14.6(8.423 .2)$ & $-12 \%$ & $88.8(75.5$ 106.7) & $50.1(40.961 .3)$ & $-44 \%$ \\
\hline Other meningitis & $17.2(10.926 .6)$ & $21.8(13.333 .6)$ & $27 \%$ & $99(86116.3)$ & $67.7(55.682 .7)$ & $-32 \%$ \\
\hline Encephalitis & $5.6(3.38 .7)$ & $5.5(3.29)$ & $-1 \%$ & $28.5(24.732 .7)$ & 17.9 (14.7 21.8) & $-37 \%$ \\
\hline Diphtheria & $0\left(\begin{array}{ll}0 & 0\end{array}\right)$ & $0\left(\begin{array}{ll}0 & 0\end{array}\right)$ & $-82 \%$ & $0.9\left(\begin{array}{lll}0 & 7.3)\end{array}\right.$ & $0.3\left(\begin{array}{ll}0 & 2.5\end{array}\right)$ & $-66 \%$ \\
\hline Whooping cough & $2.4\left(\begin{array}{ll}1.3 & 3.8\end{array}\right)$ & $0.6\left(\begin{array}{ll}0.3 & 1.1\end{array}\right)$ & $-74 \%$ & $47.8(2.3221 .4)$ & $9.3(0.640 .9)$ & $-81 \%$ \\
\hline Tetanus & $0\left(\begin{array}{ll}0 & 0\end{array}\right)$ & $0\left(\begin{array}{ll}0 & 0\end{array}\right)$ & $-69 \%$ & $3.4\left(\begin{array}{ll}0.4 & 13\end{array}\right)$ & $0.7\left(\begin{array}{ll}0.1 & 2.6\end{array}\right)$ & $-80 \%$ \\
\hline Measles & $0.8(0.31 .4)$ & $0\left(\begin{array}{ll}0 & 0.1\end{array}\right)$ & $-99 \%$ & $4(2.26 .4)$ & $1.5\left(\begin{array}{ll}0.7 & 3.9\end{array}\right)$ & $-62 \%$ \\
\hline Varicella & $16.2(8.229 .1)$ & $20.8(10.139 .2)$ & $29 \%$ & $23.3(9.263 .2)$ & $27.3(11.667 .5)$ & $18 \%$ \\
\hline Neglected tropical diseases and malaria & $8.5(2.321 .8)$ & $15.6(2.551 .2)$ & $83 \%$ & $69.6(39.7$ 120.3) & $64.9(35.1$ 120.1) & $-7 \%$ \\
\hline Malaria & $0.5\left(\begin{array}{ll}0 & 3.9\end{array}\right)$ & $0.1\left(\begin{array}{ll}0 & 0.8\end{array}\right)$ & $-75 \%$ & $0.5\left(\begin{array}{ll}0 & 3.9\end{array}\right)$ & $0.1\left(\begin{array}{ll}0 & 0.8\end{array}\right)$ & $-75 \%$ \\
\hline Leishmaniasis & $1.3\left(\begin{array}{ll}0.6 & 2.7\end{array}\right)$ & $1.4\left(\begin{array}{ll}0.6 & 2.9\end{array}\right)$ & $6 \%$ & $10.6\left(\begin{array}{ll}7.4 & 14.7)\end{array}\right.$ & $8.4(5.8$ 11.5) & $-21 \%$ \\
\hline${ }^{*}$ Cysticercosis & $0\left(\begin{array}{ll}0 & 0\end{array}\right)$ & * & * & $0.6\left(\begin{array}{ll}0 & 2.2\end{array}\right)$ & * & * \\
\hline Echinococcosis & $5.3\left(\begin{array}{ll}0.8 & 18.1)\end{array}\right)$ & 7 (1 25.6) & $32 \%$ & $15.6(2.247 .7)$ & $12.1(2.135 .5)$ & $-22 \%$ \\
\hline
\end{tabular}

\begin{abstract}
5.3 (0.8 18.1)
\end{abstract}


Table 3 Comparison of the main causes of disability-adjusted life years (DALYs) and years lived with disability (YLDs), in 1990 and 2010 , for the Spanish population (Continued)

\begin{tabular}{|c|c|c|c|c|c|c|}
\hline Dengue & $0\left(\begin{array}{llll}0 & 0\end{array}\right)$ & $0\left(\begin{array}{lll}0 & 0\end{array}\right)$ & * & $31.8(9.670 .5)$ & $30.9(12.568 .8)$ & $-3 \%$ \\
\hline Rabies & $0\left(\begin{array}{ll}0 & 0\end{array}\right)$ & $0\left(\begin{array}{ll}0 & 0\end{array}\right)$ & $13 \%$ & $5.6\left(\begin{array}{ll}2.7 & 12.4\end{array}\right)$ & $2\left(\begin{array}{ll}1.1 & 3.9\end{array}\right)$ & $-64 \%$ \\
\hline Food-borne trematodiases & $0.1\left(\begin{array}{ll}0 & 0.4\end{array}\right)$ & $0.1\left(\begin{array}{ll}0 & 0.3\end{array}\right)$ & $-31 \%$ & $0.1\left(\begin{array}{ll}0 & 0.4\end{array}\right)$ & $0.1\left(\begin{array}{ll}0 & 0.3\end{array}\right)$ & $-31 \%$ \\
\hline Other neglected tropical diseases & $1.3(0.11 .6)$ & $7(0.26 .2)$ & $425 \%$ & $4.8(2.57)$ & $11.3(3 \quad 11.7)$ & $133 \%$ \\
\hline Maternal disorders & $14.1\left(\begin{array}{ll}8.9 & 21.7\end{array}\right)$ & $12.5(7.519 .6)$ & $-11 \%$ & $37(2947.5)$ & 28.7 (21.7 37.2) & $-22 \%$ \\
\hline Maternal hemorrhage & $5.9(3.49 .5)$ & $5.3(38.5)$ & $-11 \%$ & $10.3\left(\begin{array}{ll}7 & 14\end{array}\right)$ & $8.5\left(\begin{array}{ll}6 & 11.9\end{array}\right)$ & $-17 \%$ \\
\hline Maternal sepsis & $0.3\left(\begin{array}{ll}0 & 1\end{array}\right)$ & $0.3\left(\begin{array}{ll}0 & 1\end{array}\right)$ & $-9 \%$ & $1.3\left(\begin{array}{ll}0.8 & 2.1\end{array}\right)$ & $0.9\left(\begin{array}{ll}0.5 & 1.6\end{array}\right)$ & $-33 \%$ \\
\hline Hypertensive disorders of pregnancy & $0.6\left(\begin{array}{ll}0.3 & 1.1\end{array}\right)$ & $0.7(0.31 .2)$ & $9 \%$ & $3.9(3 \quad 5.1)$ & $2.9(2.13 .8)$ & $-26 \%$ \\
\hline Obstructed labor & $1.1\left(\begin{array}{ll}0 & 3.4\end{array}\right)$ & $1.1\left(\begin{array}{ll}0 & 3.4\end{array}\right)$ & $-2 \%$ & $2(0.94 .3)$ & $1.8\left(\begin{array}{ll}0.7 & 4.2\end{array}\right)$ & $-12 \%$ \\
\hline Abortion & $2\left(\begin{array}{ll}1.1 & 3.4\end{array}\right)$ & $1.4\left(\begin{array}{ll}0.7 & 2.5\end{array}\right)$ & $-29 \%$ & $5.2(3.96 .8)$ & $3.4(2.44 .6)$ & $-35 \%$ \\
\hline Other maternal disorders & $4.1(2.66 .2)$ & $3.8(2.35 .8)$ & $-8 \%$ & $14.2\left(\begin{array}{ll}11.1 & 18.1)\end{array}\right.$ & $11.3(8.414 .4)$ & $-21 \%$ \\
\hline Neonatal disorders & $224.6(164.9300 .8)$ & $218.4\left(\begin{array}{ll}162.3 & 282.8\end{array}\right)$ & $-3 \%$ & $1,468.3(1,242.31,668.6)$ & $1,061.2(934.7 \quad 1,183)$ & $-28 \%$ \\
\hline Preterm birth complications & $96.4(66.9$ 136.9) & $109.4(75.9$ 149.6) & $13 \%$ & 788.4 (627.9 938.5) & $533(433665.9)$ & $-32 \%$ \\
\hline $\begin{array}{l}\text { Neonatal encephalopathy (birth asphyxia and } \\
\text { birth trauma) }\end{array}$ & $126.6(89.5172 .7)$ & $107(77.3143 .6)$ & $-15 \%$ & 425.4 (336.5 527.3) & $297.5(235.6375)$ & $-30 \%$ \\
\hline $\begin{array}{l}\text { Sepsis and other infectious disorders of the } \\
\text { newborn baby }\end{array}$ & $0.1\left(\begin{array}{ll}0.1 & 0.3)\end{array}\right.$ & $0.3\left(\begin{array}{ll}0.2 & 0.6\end{array}\right)$ & $140 \%$ & $110.1(60.9192 .3)$ & $111.2\left(\begin{array}{ll}60 & 197.5\end{array}\right)$ & $1 \%$ \\
\hline Other neonatal disorders & $1.4\left(\begin{array}{ll}1 & 2\end{array}\right)$ & $1.7\left(\begin{array}{ll}1.2 & 2.3)\end{array}\right.$ & $16 \%$ & $144.3(91.2 \quad 262.3)$ & $119.5(67.1179 .2)$ & $-17 \%$ \\
\hline Nutritional deficiencies & $444.6(269.2$ 712.5) & $507.8(314.2801 .1)$ & $14 \%$ & $524.2(347 \quad 797.2)$ & $590.7(394.4885)$ & $13 \%$ \\
\hline Protein-energy malnutrition & $14.3(9.321 .2)$ & $22.7\left(\begin{array}{ll}14.8 & 31.8\end{array}\right)$ & $59 \%$ & $46.2(38.557 .6)$ & 75 (52.4 93.8) & $62 \%$ \\
\hline lodine deficiency & $427.1(251.8694)$ & $480.5(285763.7)$ & $13 \%$ & $428.8(253.7 \quad 695.7)$ & $482.4(286.9765 .8)$ & $13 \%$ \\
\hline Iron-deficiency anemia & $1.1\left(\begin{array}{ll}0.5 & 2\end{array}\right)$ & $3\left(\begin{array}{ll}1 & 3.2\end{array}\right)$ & $171 \%$ & $41.8(35.454 .5)$ & $27.9\left(\begin{array}{lll}16.9 & 33.7\end{array}\right)$ & $-33 \%$ \\
\hline Other nutritional deficiencies & $2.2\left(\begin{array}{ll}1.8 & 2.8\end{array}\right)$ & $1.5\left(\begin{array}{ll}0.9 & 2\end{array}\right)$ & $-29 \%$ & $7.5(6.39 .8)$ & $5.3(3.26 .9)$ & $-29 \%$ \\
\hline $\begin{array}{l}\text { Other communicable, maternal, neonatal, and } \\
\text { nutritional disorders }\end{array}$ & 79 (38.7 149.3) & $80.1(35.6168 .3)$ & $1 \%$ & $208(161.6281 .2)$ & $225.5(162.4323 .7)$ & $8 \%$ \\
\hline Sexually transmitted diseases excluding HIV & $64.3(29.6128 .4)$ & $56.9\left(\begin{array}{ll}26 & 114\end{array}\right)$ & $-11 \%$ & $91.6(56.3157 .8)$ & $66.6(35.2123 .7)$ & $-27 \%$ \\
\hline Syphilis & $3.9(0.28 .7)$ & $4.8(0.310 .7)$ & $21 \%$ & 25.4 (15.7 39.3) & $10.9\left(\begin{array}{ll}5 & 18\end{array}\right)$ & $-57 \%$ \\
\hline Sexually transmitted chlamydial diseases & $25.3(8.760)$ & $22.4(7.653 .6)$ & $-12 \%$ & $26.7(10.161 .5)$ & $23.2(8.354 .3)$ & $-13 \%$ \\
\hline Gonococcal infection & $13.7(5.230 .2)$ & $14.5\left(\begin{array}{ll}5 & 32.7\end{array}\right)$ & $6 \%$ & $14.8\left(\begin{array}{ll}6.1 & 31.3\end{array}\right)$ & $15.1(5.633 .5)$ & $2 \%$ \\
\hline Trichomoniasis & $13.3(0.141 .8)$ & $9.2\left(\begin{array}{ll}0.1 & 29.1\end{array}\right)$ & $-31 \%$ & $13.3(0.141 .8)$ & $9.2(0.129 .1)$ & $-31 \%$ \\
\hline Other sexually transmitted diseases & $8(2.918 .1)$ & $6\left(\begin{array}{ll}2.5 & 12\end{array}\right)$ & $-25 \%$ & $11.5(6.221 .5)$ & $8.2\left(\begin{array}{ll}4.5 & 14.2\end{array}\right)$ & $-29 \%$ \\
\hline Hepatitis & $12.2\left(\begin{array}{ll}6 & 21.9\end{array}\right)$ & $12.9\left(\begin{array}{ll}6.2 & 22.8\end{array}\right)$ & $6 \%$ & $67.3(57.979)$ & 66 (55.8 77.8) & $-2 \%$ \\
\hline Acute hepatitis A & $8.3\left(\begin{array}{ll}3.9 & 14.7)\end{array}\right.$ & 7.5 (3.7 13) & $-9 \%$ & 17 (11.2 24.8) & $11\left(\begin{array}{ll}6.5 & 17.6)\end{array}\right.$ & $-35 \%$ \\
\hline Acute hepatitis B & $2.8\left(\begin{array}{ll}0.2 & 9.2)\end{array}\right.$ & $3.4\left(\begin{array}{ll}0.2 & 10.6\end{array}\right)$ & $21 \%$ & $42.8(26.355 .3)$ & $24(12.6 \quad 43.4)$ & $-44 \%$ \\
\hline
\end{tabular}


Table 3 Comparison of the main causes of disability-adjusted life years (DALYs) and years lived with disability (YLDs), in 1990 and 2010 , for the Spanish population (Continued)

\begin{tabular}{|c|c|c|c|c|c|c|}
\hline Acute hepatitis C & $1(0.22 .1)$ & $2\left(\begin{array}{ll}0.4 & 4.1)\end{array}\right.$ & $87 \%$ & $7.6(2.815 .8)$ & $31(10.553 .4)$ & $308 \%$ \\
\hline Leprosy & $0.1\left(\begin{array}{ll}0 & 0.1\end{array}\right)$ & $0\left(\begin{array}{ll}0 & 0\end{array}\right)$ & $-87 \%$ & $0.1\left(\begin{array}{ll}0 & 0.1\end{array}\right)$ & $0\left(\begin{array}{ll}0 & 0\end{array}\right)$ & $-87 \%$ \\
\hline Other infectious diseases & $2.5\left(\begin{array}{ll}0 & 0.3\end{array}\right)$ & $10.3\left(\begin{array}{ll}0 & 0.4\end{array}\right)$ & $314 \%$ & 49 (37.6 81.3) & $92.8(39.3114 .7)$ & $89 \%$ \\
\hline Non-communicable diseases & $38,528.9(32,278.245,503.6)$ & $48,269.1(40,257.656,616.6)$ & $25 \%$ & $86,260(79,823.193,301.2)$ & $95,239.4(87,522.210,3475.6)$ & $10 \%$ \\
\hline Neoplasms & $474.2(349.1630 .6)$ & $816.4(597.6$ 1074.5) & $72 \%$ & $16,917.3(16,102.7 \quad 17,858.3)$ & $19,304.5(18,00320,302.6)$ & $14 \%$ \\
\hline Esophageal cancer & $3.2(1.65 .3)$ & $3.6(1.8 \quad 6.2)$ & $10 \%$ & 413.9 (310.3 535.2) & 392.1 (290.6 538.8) & $-5 \%$ \\
\hline Stomach cancer & $24.3(15.335 .6)$ & $21.2(12.933)$ & $-13 \%$ & $1,443.2(1,131 \quad 1,931.8)$ & $1,007.9(7651,402.6)$ & $-30 \%$ \\
\hline Liver cancer & 6.5 (3.9 9.8) & $9.6(5.814 .8)$ & $47 \%$ & $720(581.5818 .5)$ & $846.8(712.5$ 1,044.6) & $18 \%$ \\
\hline Liver cancer secondary to hepatitis B & $0.9(0.31 .8)$ & $1.3\left(\begin{array}{lll}0.4 & 2.4\end{array}\right)$ & $38 \%$ & $100.9(80.1116 .3)$ & $119.2(97.8$ 152.4) & $18 \%$ \\
\hline Liver cancer secondary to hepatitis C & $2.8\left(\begin{array}{ll}1.5 & 4.7\end{array}\right)$ & $3.9(26.6)$ & $40 \%$ & 287.9 (236.3 332.2) & $331.9(277.3427 .1)$ & $15 \%$ \\
\hline Liver cancer secondary to alcohol use & $2.2\left(\begin{array}{ll}1.1 & 3.7\end{array}\right)$ & $3.6(1.95 .9)$ & $64 \%$ & $253(201.7$ 291.3) & $301(253$ 381) & $19 \%$ \\
\hline Other liver cancer & $0.6\left(\begin{array}{ll}0.1 & 1.4\end{array}\right)$ & $0.8(0.11 .6)$ & $26 \%$ & $78.2(62.990 .4)$ & 94.7 (74.6 116.3) & $21 \%$ \\
\hline Larynx cancer & $14\left(\begin{array}{ll}7.3 & 24.9\end{array}\right)$ & $12.3\left(\begin{array}{ll}6.5 & 23.3\end{array}\right)$ & $-12 \%$ & 498.7 (252.2 859.6) & $373.9(202.3685 .2)$ & $-25 \%$ \\
\hline Trachea, bronchus, and lung cancers & $34.1(22.349 .5)$ & $45.5(25.964 .2)$ & $34 \%$ & $3,138.8(2,541.8 \quad 3,982.3)$ & $3,686.7(2,408.14,161.1)$ & $17 \%$ \\
\hline Breast cancer & $122.7(80.8$ 187.9) & 188.1 (120.8 277.2) & $53 \%$ & $1,496.8(1,400.8 \quad 1,611.5)$ & 1,469.4 (1,340.6 1,619.2) & $-2 \%$ \\
\hline Cervical cancer & $5.4(2.98 .7)$ & $5.2\left(\begin{array}{ll}2.7 & 8.6\end{array}\right)$ & $-4 \%$ & 226.8 (143.3 321.6) & $212.3(135.6306 .1)$ & $-6 \%$ \\
\hline Uterine cancer & 10.9 (5.7 20.1) & $13.6(5.723 .1)$ & $24 \%$ & $174.1(100.8316 .1)$ & $194.6(93.5$ 271.9) & $12 \%$ \\
\hline Prostate cancer & $40.2(2760)$ & $120.3(78.9185 .9)$ & $199 \%$ & 609 (373.5 941.4) & $939.3(572.7$ 1,489.8) & $54 \%$ \\
\hline Colon and rectum cancers & $63.1(44.8$ 85.4) & $128.7(90.3171 .7)$ & $104 \%$ & $1,675.7(1,476.61,895.5)$ & $2,452.4(2,019.5 \quad 2,711)$ & $46 \%$ \\
\hline Mouth cancer & $12.4(8.217 .8)$ & $13.8(8.920 .3)$ & $12 \%$ & $307.3(257.8337 .4)$ & $296.6(250.1367 .2)$ & $-3 \%$ \\
\hline Nasopharynx cancer & $0.9\left(\begin{array}{ll}0.3 & 1.9\end{array}\right)$ & $1\left(\begin{array}{ll}0.3 & 2\end{array}\right)$ & $5 \%$ & $70.2(40.194 .4)$ & $63.2(40.488 .2)$ & $-10 \%$ \\
\hline Cancer of other part of pharynx and oropharynx & $4.1(2 \quad 7)$ & $5.8(2.89 .4)$ & $42 \%$ & $172.5(100.3251 .3)$ & 211.9 (125.8 299.5) & $23 \%$ \\
\hline Gallbladder and biliary tract cancer & $3.6(1.96 .1)$ & $5.1(2.59)$ & $41 \%$ & 253.9 (169.6 370.5) & $274.3(193414.1)$ & $8 \%$ \\
\hline Pancreatic cancer & $3.9(26.5)$ & $6.3(3.410 .4)$ & $63 \%$ & $627.8(480.8812 .9)$ & $881.8(646.5$ 1120.7) & $40 \%$ \\
\hline Malignant melanoma of skin & $4.2(2.37 .9)$ & $8.6\left(\begin{array}{ll}4.7 & 13.7\end{array}\right)$ & $103 \%$ & $140.1(94.4223 .2)$ & $207.1(133$ 294.9) & $48 \%$ \\
\hline Non-melanoma skin cancer & $15.6\left(\begin{array}{ll}10.7 & 21.9)\end{array}\right.$ & $38(25.952 .8)$ & $143 \%$ & $89.4\left(\begin{array}{ll}55.1 & 125.3\end{array}\right)$ & $110.9(80.5165 .1)$ & $24 \%$ \\
\hline Ovarian cancer & $6.2(3.69 .8)$ & $9.4(5.214 .5)$ & $52 \%$ & 336.7 (252.6 453.1) & $425.2(287.4561 .8)$ & $26 \%$ \\
\hline Testicular cancer & $0.8(0.21 .6)$ & $1.4\left(\begin{array}{ll}0.5 & 2.9\end{array}\right)$ & $86 \%$ & $22.9\left(\begin{array}{ll}13.7 & 34.8\end{array}\right)$ & $22.7(12.932 .9)$ & $-1 \%$ \\
\hline Kidney and other urinary organ cancers & $5.6(3.18 .6)$ & $17.7(10.230 .6)$ & $218 \%$ & 255.5 (178.9 327.2) & 592.6 (435.2958.7) & $132 \%$ \\
\hline Bladder cancer & $31.7(21.7$ 43.9) & $49.8(32.269 .6)$ & $57 \%$ & $617(491.5789 .2)$ & $704.1(508.2824 .7)$ & $14 \%$ \\
\hline Brain and nervous system cancers & $11.5\left(\begin{array}{ll}6.6 & 17.9\end{array}\right)$ & $17.4\left(\begin{array}{ll}8.7 & 27.2\end{array}\right)$ & $51 \%$ & $716.6(488.11,011.1)$ & $848.3(492.41,050.7)$ & $18 \%$ \\
\hline Thyroid cancer & $2.5(1.34 .4)$ & $4.1(2.26 .8)$ & $63 \%$ & $51.9(40.566 .8)$ & $61.5(45.7$ 78.9) & $18 \%$ \\
\hline Hodgkin s disease & $2.4\left(\begin{array}{ll}1 & 4.2\end{array}\right)$ & $2.1(0.93 .9)$ & $-11 \%$ & $112.8(73.1166 .8)$ & $72.3\left(\begin{array}{ll}47.9 & 109.7)\end{array}\right.$ & $-36 \%$ \\
\hline
\end{tabular}


Table 3 Comparison of the main causes of disability-adjusted life years (DALYs) and years lived with disability (YLDs), in 1990 and 2010 , for the Spanish population (Continued)

\begin{tabular}{|c|c|c|c|c|c|c|}
\hline Non-Hodgkin s lymphoma & $9.4(6 \quad 13.8)$ & $16.1\left(\begin{array}{ll}10 & 23.9\end{array}\right)$ & $72 \%$ & $417.3(366.4$ 493.7) & $503.5(364.3578 .3)$ & $21 \%$ \\
\hline Multiple myeloma & $5.4(2.89)$ & $8.3(4.313 .5)$ & $55 \%$ & $212.7\left(\begin{array}{lll}146.8 & 305.2\end{array}\right)$ & $275.6(182.9388 .8)$ & $30 \%$ \\
\hline Leukemia & $10.4\left(\begin{array}{ll}6.7 & 15.4\end{array}\right)$ & $16.1\left(\begin{array}{ll}10 & 23.5\end{array}\right)$ & $55 \%$ & $705.9(575.5880)$ & $688.4(544.1817)$ & $-2 \%$ \\
\hline Other neoplasms & $19.2(12.428 .5)$ & $47.2(3069.8)$ & $145 \%$ & $1,409.8(1,108.1 \quad 1,845.1)$ & $1,489.3(1,190.52,016.9)$ & $6 \%$ \\
\hline Cardiovascular and circulatory diseases & $1,911.6(1,475.92,423)$ & $2,864.9(2,268.9 \quad 3,499.4)$ & $50 \%$ & $20,076.7(18,998.520,904.2)$ & $17,802(16,834.219,181.8)$ & $-11 \%$ \\
\hline Rheumatic heart disease & $125.4(77.4198 .2)$ & $139.1(93.2207 .5)$ & $11 \%$ & $823.4(745.2933 .8)$ & $507.3(429.5599)$ & $-38 \%$ \\
\hline Ischemic heart disease & $737.6(477.91108)$ & $974.8(6361420.7)$ & $32 \%$ & $9,202.7(8,705.19,952.4)$ & $8,055.8(7,462.7 \quad 8,876.3)$ & $-12 \%$ \\
\hline Cerebrovascular disease & $337.1(278.1393 .5)$ & $640.5(529.3755 .2)$ & $90 \%$ & $6,437.3(5,648.16,783.7)$ & $4,752.8(4,252.95,829.1)$ & $-26 \%$ \\
\hline Ischemic stroke & $271.4(225318.7)$ & $515.9(426.9609 .4)$ & $90 \%$ & $4,130.9(3,546.54,389.3)$ & $3,011(2,635.24,183.6)$ & $-27 \%$ \\
\hline Hemorrhagic and other non-ischemic stroke & $65.6(54.377 .7)$ & $124.6(102.1147 .8)$ & $90 \%$ & $2,306.5(2,058.82,601.6)$ & $1,741.8(1,502.31,936)$ & $-24 \%$ \\
\hline Hypertensive heart disease & $28.3(17.342 .8)$ & $46.5(28.871 .4)$ & $65 \%$ & $531.9(432.3671 .4)$ & $613(490.4764 .1)$ & $15 \%$ \\
\hline Cardiomyopathy and myocarditis & $17.5(10.327 .4)$ & $26.6(16.340 .9)$ & $52 \%$ & $537.7(496.6569 .2)$ & $723.9(634857.1)$ & $35 \%$ \\
\hline Atrial fibrillation and flutter & $278.3\left(\begin{array}{ll}186.1 & 398\end{array}\right)$ & $460.9(306.5658)$ & $66 \%$ & $363.2(269.6484 .1)$ & $849.2(631.81127 .7)$ & $134 \%$ \\
\hline Aortic aneurysm & $0\left(\begin{array}{ll}0 & 0\end{array}\right)$ & $0\left(\begin{array}{ll}0 & 0\end{array}\right)$ & * & 261.2 (199.7 339.1) & $308.2(224.6403 .1)$ & $18 \%$ \\
\hline Peripheral vascular disease & $39.5(20.170 .8)$ & $60.7(30.8$ 109.7) & $54 \%$ & $83.5(54.6124 .9)$ & $174.6(116.3253 .8)$ & $109 \%$ \\
\hline Endocarditis & $4.2(2.46 .8)$ & $7.4(4.511 .6)$ & $75 \%$ & $74.6\left(\begin{array}{ll}64 & 86.9\end{array}\right)$ & $76.3(59.9$ 88) & $2 \%$ \\
\hline Other cardiovascular and circulatory diseases & $343.8(182.1561 .1)$ & $508.7(275.7807 .5)$ & $48 \%$ & $1,761.1(1,592.81,992.2)$ & $1,740.9(1,496.82,046.5)$ & $-1 \%$ \\
\hline Chronic respiratory diseases & $1,935.1(1,247 \quad 2,764.6)$ & $22,17.7(14,39.431,23.6)$ & $15 \%$ & $5,623.2(4,907.36,436.1)$ & $5,260.3(4,434.96,184.9)$ & $-6 \%$ \\
\hline Chronic obstructive pulmonary disease & $860.7(5,23.1 \quad 1,276.8)$ & 10,99.1 (683 15,94.7) & $28 \%$ & $3,030.1(2,586.43,515.9)$ & $2,916(2,423.7 \quad 3,483.1)$ & $-4 \%$ \\
\hline Pneumoconiosis & $8.3(5.312)$ & $15.9\left(\begin{array}{ll}10 & 23.7\end{array}\right)$ & $92 \%$ & $195.7\left(\begin{array}{ll}134.5 & 283\end{array}\right)$ & $158.8(112.5$ 223.2) & $-19 \%$ \\
\hline Asthma & $749.4(411.4$ 1,203.2) & $791.4(4431,253.1)$ & $6 \%$ & $969.4(632.41423 .6)$ & $917.8(564.5$ 1377.3) & $-5 \%$ \\
\hline Interstitial lung disease and pulmonary sarcoidosis & $8.9(5.913 .3)$ & $16.1(10.424 .3)$ & $81 \%$ & $158.8\left(\begin{array}{ll}118.1251 .5\end{array}\right)$ & $262.6(181.8373)$ & $65 \%$ \\
\hline Other chronic respiratory diseases & $307.9\left(\begin{array}{ll}187 & 458.9\end{array}\right)$ & $295.2\left(\begin{array}{lll}183.7 & 431.1\end{array}\right)$ & $-4 \%$ & 1,269.2 (988 1,483.8) & 1,005.2(819.7 1,264) & $-21 \%$ \\
\hline Cirrhosis of the liver & $34.4(21.949 .6)$ & $37.6(24.354)$ & $9 \%$ & $2,358.1(1,931.42,609.8)$ & $1,772.7(1585.92,231.2)$ & $-25 \%$ \\
\hline Cirrhosis of the liver secondary to hepatitis B & $1.3\left(\begin{array}{ll}0.5 & 2.5\end{array}\right)$ & $1.3\left(\begin{array}{lll}0.5 & 2.6\end{array}\right)$ & $4 \%$ & $89.4(70.2105 .5)$ & $67.9\left(\begin{array}{ll}57 & 86.1\end{array}\right)$ & $-24 \%$ \\
\hline Cirrhosis of the liver secondary to hepatitis C & $13.2(7.421 .5)$ & $14.4\left(\begin{array}{ll}7.7 & 23.3\end{array}\right)$ & $9 \%$ & $895(711.61049 .2)$ & $687.1(582870.4)$ & $-23 \%$ \\
\hline Cirrhosis of the liver secondary to alcohol use & $15.8(8.925)$ & $17.5(9.928 .4)$ & $11 \%$ & 1,105.7 (847.2 1295.5) & $830.5(694.91,084.7)$ & $-25 \%$ \\
\hline Other cirrhosis of the liver & $4.1(26.9)$ & $4.3(2.17 .6)$ & $4 \%$ & $268(211.1313 .4)$ & $187.2\left(\begin{array}{ll}156.9 & 238.2\end{array}\right)$ & $-30 \%$ \\
\hline Digestive diseases (except cirrhosis) & $423.2(248.1730 .7)$ & $492.4(313.7$ 755.8) & $16 \%$ & $1,683(1,452.62,012.8)$ & $1,860(1,610.12,164.5)$ & $11 \%$ \\
\hline Peptic ulcer disease & $34(17.964 .8)$ & $21.8\left(\begin{array}{ll}11 & 46.2\end{array}\right)$ & $-36 \%$ & $251.8(202.3293 .9)$ & $116.2(95164.7)$ & $-54 \%$ \\
\hline Gastritis and duodenitis & $29.9(19.344 .6)$ & $38.8(25.856 .3)$ & $30 \%$ & 46.9 (33.4 62.6) & $46.5(32.165)$ & $-1 \%$ \\
\hline Appendicitis & $7.8\left(\begin{array}{ll}2.8 & 16.7\end{array}\right)$ & $8.3(3$ 17.8) & $6 \%$ & 43.7 (30.9 65.7) & 37.7 (22.8 52.5) & $-14 \%$ \\
\hline
\end{tabular}


Table 3 Comparison of the main causes of disability-adjusted life years (DALYs) and years lived with disability (YLDs), in 1990 and 2010 , for the Spanish population (Continued)

\begin{tabular}{|c|c|c|c|c|c|c|}
\hline $\begin{array}{l}\text { Paralytic ileus and intestinal obstruction } \\
\text { without hernia }\end{array}$ & $0.6\left(\begin{array}{ll}0 & 2.7\end{array}\right)$ & $0.6\left(\begin{array}{ll}0 & 2.6\end{array}\right)$ & $3 \%$ & $123.3(96.4175 .4)$ & $169.4(106.6226 .9)$ & $37 \%$ \\
\hline Inguinal or femoral hernia & $28.3(6.684)$ & 28.9 (5.7 81.8) & $2 \%$ & $55.8(33.9110 .3)$ & 48.7 (25.4 101.4) & $-13 \%$ \\
\hline Non-infective inflammatory bowel disease & $205.8(88.9434 .4)$ & 214.3 (109.5 390.4) & $4 \%$ & $243.3(127.2473 .9)$ & $257(151.9435 .7)$ & $6 \%$ \\
\hline Vascular disorders of intestine & $1(0.22 .4)$ & $1.6\left(\begin{array}{ll}0.3 & 3.9\end{array}\right)$ & $70 \%$ & $232.9(117.1468 .5)$ & $313.5(166604)$ & $35 \%$ \\
\hline Gall bladder and bile duct disease & $36.4(24.5$ 51.6) & $45.6(30.764 .3)$ & $25 \%$ & 189.2 (161.3 229.6) & 207.8 (164.8 260.5) & $10 \%$ \\
\hline Pancreatitis & $16.3(5.838 .3)$ & $37.2(12.8$ 83.9) & $128 \%$ & $224.4(164.8$ 275.8) & $228.1\left(\begin{array}{ll}177 & 303.9\end{array}\right)$ & $2 \%$ \\
\hline Other digestive diseases & $63.2\left(\begin{array}{ll}37 & 108.4\end{array}\right)$ & $95.3(60.6146 .9)$ & $51 \%$ & $271.7(228$ 356.4) & $435.1(337.6529 .1)$ & $60 \%$ \\
\hline Neurological disorders & $3,407(2,631.94,321.9)$ & $4,627.9(3,616.35,804.5)$ & $36 \%$ & $4,581(3,789.15,481.9)$ & 7,372.4 (6,127.5 8,666.4) & $61 \%$ \\
\hline Alzheimer's disease and other dementias & $858.3(612.71,136)$ & $1,640.7(1,185$ 2,196.8) & $91 \%$ & $1,433.9(1,138.41,754.3)$ & $3,518(2707.84,334.5)$ & $145 \%$ \\
\hline Parkinson's disease & $71.8\left(\begin{array}{ll}4 & 114.3\end{array}\right)$ & 115.7 (72.9 189) & $61 \%$ & 230.4 (180.8 300.8) & $390(290.8496 .1)$ & $69 \%$ \\
\hline Epilepsy & $292(215.3381 .1)$ & $324(242.9425 .2)$ & $11 \%$ & 399.4 (320.1 491.9) & $432.1(343538.1)$ & $8 \%$ \\
\hline Multiple sclerosis & 37.7 (26.5 51) & $54.5(37.374 .1)$ & $45 \%$ & $82.1(67.698 .9)$ & $109(86.3133 .8)$ & $33 \%$ \\
\hline Migraine & $1,893.5(1,198.12,731.1)$ & $2,146(1,339.7 \quad 3,101.9)$ & $13 \%$ & 1893.5 (1198.1 2731.1) & 2146 (1339.7 3101.9) & $13 \%$ \\
\hline Tension-type headache & $117.1(65.8188)$ & $144.1(82.7233 .9)$ & $23 \%$ & $117.1(65.8188)$ & $144.1(82.7233 .9)$ & $23 \%$ \\
\hline Other neurological disorders & $136.7(102.7176 .2)$ & 202.8 (153.7 261.7) & $48 \%$ & 424.5 (336.1 567.1) & $633.1(480.2802 .4)$ & $49 \%$ \\
\hline Mental and behavioral disorders & $10,177.2(8,247.312,325.7)$ & $12,464.1(10,170.815,032.3)$ & $22 \%$ & $10,528.7(8,600.512,716.4)$ & $12,917.2(10,630.615,432.4)$ & $23 \%$ \\
\hline Schizophrenia & $771.1(443.1$ 1,144.3) & 1,052.2 (583.9 1,591.4) & $36 \%$ & $781.6(453.6$ 1,153.9) & $1,074.7(606.8$ 1,614.1) & $37 \%$ \\
\hline Alcohol use disorders & $691(381.21156 .4)$ & $757.1(401.2$ 1,235.8) & $10 \%$ & $797(482.4$ 1,257.7) & $882.1(514.8$ 1,385.4) & $11 \%$ \\
\hline Drug use disorders & $1,644.7(1,176.5 \quad 2,156.1)$ & $1,784.5(1,277.6 \quad 2,334.5)$ & $9 \%$ & $1,873.5(1,391 \quad 2,416.5)$ & $2,076(1,536.1 \quad 2,677.5)$ & $11 \%$ \\
\hline Opioid use disorders & $746.5(502.9$ 1003.1) & $844.4(571.9$ 1,134.8) & $13 \%$ & $886.9(629.1$ 1,165.1) & $1,016.2(728.8$ 1,339.5) & $15 \%$ \\
\hline Cocaine use disorders & 195.6 (112.9 306.5) & 216.7 (124.3 335) & $11 \%$ & 199.3 (117.2 313.6) & 218.6 (124.8 338.9) & $10 \%$ \\
\hline Amphetamine use disorders & $155.2(86.9249 .2)$ & $162.1(91.4255 .5)$ & $4 \%$ & $157.2(88.8$ 251.8) & $164.5(92.3262 .9)$ & $5 \%$ \\
\hline Cannabis use disorders & $213.6(107.1377 .3)$ & 201.5 (100.3 367.1) & $-6 \%$ & 213.6 (107.1 377.3) & 201.5 (100.3 367.1) & $-6 \%$ \\
\hline Other drug use disorders & $333.8(205.6499 .4)$ & 359.8 (226.7 523.5) & $8 \%$ & 416.4 (280.9 592.2) & 475.1 (327 669.2) & $14 \%$ \\
\hline Unipolar depressive disorders & 4,011.1 (2,829.7 5,395.6) & $5,308.1(3,910.9$ 7,025.8) & $32 \%$ & 4,011.1 (2,829.7 5,395.6) & $5,308.1(3,910.9$ 7,025.8) & $32 \%$ \\
\hline Major depressive disorder & 3,352.4 (2,332.1 4,626.6) & 4,521.8 (3,227.7 6,093.6) & $35 \%$ & $3,352.4(2,332.1 \quad 4,626.6)$ & 4,521.8 (3,227.7 6,093.6) & $35 \%$ \\
\hline Dysthymia & $658.7(426.3922 .7)$ & $786.3(508.1 \quad 1,094.9)$ & $19 \%$ & $658.7(426.3922 .7)$ & $786.3(508.1$ 1,094.9) & $19 \%$ \\
\hline Bipolar affective disorder & $696.1(425.11051)$ & $835.3(510.6$ 1,251.2) & $20 \%$ & $696.1(425.11051)$ & $835.3(510.61251 .2)$ & $20 \%$ \\
\hline Anxiety disorders & 1,213.6 (826.9 1,673.9) & $1331.1(906.81,856.4)$ & $10 \%$ & 1,213.6 (826.9 1,673.9) & 1,331.1 (906.8 1,856.4) & $10 \%$ \\
\hline Eating disorders & $296.4(171.5476 .8)$ & 570.1 (334.2 924.9) & $92 \%$ & 298.7 (174.6 479.3) & 574.6 (339.7 929.5) & $92 \%$ \\
\hline Pervasive development disorders & $399.8(269.4578 .2)$ & $452.2(302.8$ 648.9) & $13 \%$ & 399.8 (269.4 578.2) & $452.2(302.8$ 648.9) & $13 \%$ \\
\hline Autism & $188.8(122.7275 .7)$ & 216.3 (140.4 317.6) & $15 \%$ & 188.8 (122.7 275.7) & 216.3 (140.4 317.6) & $15 \%$ \\
\hline
\end{tabular}


Table 3 Comparison of the main causes of disability-adjusted life years (DALYs) and years lived with disability (YLDs), in 1990 and 2010 , for the Spanish population (Continued)

\begin{tabular}{|c|c|c|c|c|c|c|}
\hline Asperger s syndrome & $211.1(134.8319 .5)$ & $235.8(154.4352 .7)$ & $12 \%$ & $211.1(134.8319 .5)$ & $235.8(154.4352 .7)$ & $12 \%$ \\
\hline Childhood behavioral disorders & $291.8(171.3450 .9)$ & $209.7(120.2323 .4)$ & $-28 \%$ & $291.8(171.3450 .9)$ & $209.7(120.2323 .4)$ & $-28 \%$ \\
\hline Attention-deficit hyperactivity disorder & $27(15.243 .6)$ & $20.8(11.833 .8)$ & $-23 \%$ & $27(15.243 .6)$ & $20.8(11.833 .8)$ & $-23 \%$ \\
\hline Conduct disorder & $264.7(151419.6)$ & $188.9\left(\begin{array}{ll}105 & 299.2\end{array}\right)$ & $-29 \%$ & $264.7(151419.6)$ & $188.9(105299.2)$ & $-29 \%$ \\
\hline Idiopathic intellectual disability & $92(53.5142 .5)$ & $74.1(38$ 123.4) & $-19 \%$ & $92(53.5142 .5)$ & $74.1(38$ 123.4) & $-19 \%$ \\
\hline Other mental and behavioral disorders & $69.5\left(\begin{array}{ll}41 & 108.2\end{array}\right)$ & $89.7(52143.5)$ & $29 \%$ & $73.4(44.8$ 112.2) & $99.2(60.1$ 152.2) & $35 \%$ \\
\hline Diabetes, urogenital, blood, and endocrine diseases & $4,245.7(3,050.25,693.7)$ & $5,381.9(3,956.87,145.9)$ & $27 \%$ & $7,013.4(5,814.58,532.3)$ & $8,414.8(6,968.81,0395.6)$ & $20 \%$ \\
\hline Diabetes mellitus & 2,207.8(1461.1 3173) & $2,807.9(1,906.54,022.5)$ & $27 \%$ & $3,490.7(2,731.4 \quad 4,487.2)$ & $3,954.2(3,014.95,187.7)$ & $13 \%$ \\
\hline Acute glomerulonephritis & $0\left(\begin{array}{ll}0 & 0.1\end{array}\right)$ & $0\left(\begin{array}{ll}0 & 0\end{array}\right)$ & $-9 \%$ & $3.8(2.46 .7)$ & $2.5\left(\begin{array}{ll}1.1 & 3.9\end{array}\right)$ & $-34 \%$ \\
\hline Chronic kidney diseases & $537.1(370.6805 .8)$ & $770.3(538.4$ 1101) & $43 \%$ & $1,371.7(1,146.51,649.9)$ & $1,590.2(1,313.1 \quad 1,967.6)$ & $16 \%$ \\
\hline Chronic kidney disease due to diabetes mellitus & $80.8(52.9117 .2)$ & $119.3(78.1172 .1)$ & $48 \%$ & $214.8\left(\begin{array}{lll}173.7 & 261.2\end{array}\right)$ & $242(190.6308 .7)$ & $13 \%$ \\
\hline Chronic kidney disease due to hypertension & $99.2(66.2147 .1)$ & $143.9(97.4204 .3)$ & $45 \%$ & $264.7(219.2322 .4)$ & $315.3(257.2393 .6)$ & $19 \%$ \\
\hline Chronic kidney disease unspecified & $357.1(240.4547 .6)$ & $507.1(352.2735)$ & $42 \%$ & $892.2(740.51101 .4)$ & $1,032.9(841.51,303.6)$ & $16 \%$ \\
\hline Urinary diseases and male infertility & $395.5(254.4603 .1)$ & $541.2(347.1812 .7)$ & $37 \%$ & $604.2(451.8809 .5)$ & $963.5(717.5$ 1,265.4) & $59 \%$ \\
\hline $\begin{array}{l}\text { Tubulointerstitial nephritis, pyelonephritis, and } \\
\text { urinary tract infections }\end{array}$ & $5.9(2.611 .2)$ & $7.4(3.514 .3)$ & $27 \%$ & $156.5(124.4229 .1)$ & $328.8(177.1474 .9)$ & $110 \%$ \\
\hline Urolithiasis & $50(27.381 .3)$ & $72.6\left(\begin{array}{ll}40.1 & 114.7\end{array}\right)$ & $45 \%$ & 59.1 (36 91.8) & $78.8\left(\begin{array}{ll}46.4 & 122.1\end{array}\right)$ & $33 \%$ \\
\hline Benign prostatic hyperplasia & $319.6(201.5$ 487.1) & $431.8(270.1654 .1)$ & $35 \%$ & $319.6(201.5487 .1)$ & $431.8(270.1654 .1)$ & $35 \%$ \\
\hline Male infertility & $3.2(1.27 .1)$ & $4.2(1.69)$ & $32 \%$ & $3.2(1.27 .1)$ & $4.2(1.69)$ & $32 \%$ \\
\hline Other urinary diseases & $16.9(9.5 \quad 27.4)$ & $25.1(14.140 .3)$ & $49 \%$ & $65.9(51.686)$ & $119.8(87.9155 .5)$ & $82 \%$ \\
\hline Gynecological diseases & $464.2(258.8797)$ & $585.2(325.2999)$ & $26 \%$ & $468.9(263.7801 .4)$ & $590.4(3301,004)$ & $26 \%$ \\
\hline Uterine fibroids & $96.8(43.5$ 190.7) & $139.2(60.2268 .9)$ & $44 \%$ & $97.2(43.9$ 191.1) & $139.7(60.8269 .4)$ & $44 \%$ \\
\hline Polycystic ovarian syndrome & $142.4(66.4265 .2)$ & $170.3(77.9324 .2)$ & $20 \%$ & $142.4(66.4265 .2)$ & $170.3(77.9324 .2)$ & $20 \%$ \\
\hline Female infertility & $2.5(0.95 .4)$ & $3.1\left(\begin{array}{ll}1.1 & 6.7\end{array}\right)$ & $24 \%$ & $2.5(0.95 .4)$ & $3.1\left(\begin{array}{ll}1.1 & 6.7\end{array}\right)$ & $24 \%$ \\
\hline Endometriosis & $27.1(952.7)$ & $34.3(12.466 .5)$ & $27 \%$ & $27.1(952.7)$ & $34.3(12.466 .5)$ & $27 \%$ \\
\hline Genital prolapse & $123.8(49.5 \quad 259.5)$ & $156.3\left(\begin{array}{lll}62.6 & 339.3)\end{array}\right.$ & $26 \%$ & $124.2(49.9259 .9)$ & $156.7\left(\begin{array}{ll}63 & 339.7\end{array}\right)$ & $26 \%$ \\
\hline Premenstrual syndrome & 71.7 (0 200.2) & 82.1 (0 213.6) & $14 \%$ & 71.7 (0 200.2) & $82.1\left(\begin{array}{ll}0 & 213.6\end{array}\right)$ & $14 \%$ \\
\hline Other gynecological diseases & $0\left(\begin{array}{ll}0 & 0\end{array}\right)$ & $0\left(\begin{array}{ll}0 & 0\end{array}\right)$ & * & $3.9(3.44 .6)$ & $4.2(3.35 .4)$ & $10 \%$ \\
\hline Hemoglobinopathies and hemolytic anemia & $498.7(296.21080 .4)$ & $477.4(291.8935)$ & $-4 \%$ & $599.4(393.7$ 1,169.3) & $540.7(350.5997 .4)$ & $-10 \%$ \\
\hline Thalassemia & $161.4\left(\begin{array}{ll}102.1 & 247.1)\end{array}\right.$ & $156.1(99.3254 .6)$ & $-3 \%$ & $174.9(115.5260 .7)$ & $162.3(105.8$ 260.9) & $-7 \%$ \\
\hline Sickle cell disorders & $309.9(171.6832 .4)$ & $296.2(172.1703 .8)$ & $-4 \%$ & $338.4(199.5859 .6)$ & $309.3(184.8715 .3)$ & $-9 \%$ \\
\hline G6PD deficiency & $24.8(11.793 .6)$ & $18.3(9.358 .9)$ & $-26 \%$ & $32.5(19$ 101.1) & $22.5(13.262 .6)$ & $-31 \%$ \\
\hline Other hemoglobinopathies and hemolytic anemia & $2.5(0.51 .8)$ & $6.7\left(\begin{array}{ll}0.9 & 9.3\end{array}\right)$ & $169 \%$ & $53.6(36.264 .7)$ & $46.6(31.479 .2)$ & $-13 \%$ \\
\hline
\end{tabular}


Table 3 Comparison of the main causes of disability-adjusted life years (DALYs) and years lived with disability (YLDs), in 1990 and 2010 , for the Spanish population (Continued)

\begin{tabular}{|c|c|c|c|c|c|c|}
\hline $\begin{array}{l}\text { Other endocrine, nutritional, blood, and } \\
\text { immune disorders }\end{array}$ & $142.4(83.1228 .4)$ & $199.8(115.9362)$ & $40 \%$ & $474.8(329.2710 .6)$ & $773.4(529.7$ 1,539.3) & $63 \%$ \\
\hline Musculoskeletal disorders & $11,220.4(, 8585.214,277.6)$ & $14,278.5(10,905.8 \quad 17,775.5)$ & $27 \%$ & $11,469.4(88,29.8$ 14,558.2) & $14,654.1(11,291.8$ 18,160.2) & $28 \%$ \\
\hline Rheumatoid arthritis & $477.5(345.2636 .5)$ & $665.5(465.5881)$ & $39 \%$ & $519.4(384.9684 .3)$ & 710.1 (509.5 925.6) & $37 \%$ \\
\hline Osteoarthritis & $1,055.3(648.4 \quad 1,604.3)$ & $1,407.1(877.8 \quad 2,066.2)$ & $33 \%$ & $1,055.3(648.41,604.3)$ & $1,407.1(877.8 \quad 2,066.2)$ & $33 \%$ \\
\hline Low back and neck pain & $7,363.4(5,148.29,886.3)$ & $9,178.1(6,357.5 \quad 12,295.1)$ & $25 \%$ & 7,363.4 (5,148.2 9,886.3) & $9,178.1(6,357.51,2295.1)$ & $25 \%$ \\
\hline Low back pain & $4,784.5(3,305.8 \quad 6,484.6)$ & $6,011.7(4,104.58,080.9)$ & $26 \%$ & 4,784.5 (3,305.8 6,484.6) & $6,011.7(4,104.58,080.9)$ & $26 \%$ \\
\hline Neck pain & $2,578.9(1,769.5 \quad 3,608.8)$ & $3,166.3(2,193.9 \quad 4,438)$ & $23 \%$ & $2,578.9(1,769.5 \quad 3,608.8)$ & $3,166.3(2,193.9 \quad 4,438)$ & $23 \%$ \\
\hline Gout & $21.6(13.432 .1)$ & $30.2(1945.1)$ & $40 \%$ & $21.6(13.432 .1)$ & $30.2(1945.1)$ & $40 \%$ \\
\hline Other musculoskeletal disorders & 2,302.7 (1,585.5 3,228.6) & $2,997.7(2,089.4$ 4,136.6) & $30 \%$ & $2,509.7(1,803.7 \quad 3,433.4)$ & $3,328.7(2,440.8 \quad 4,471.4)$ & $33 \%$ \\
\hline Other non-communicable diseases & $4,700.2(3,172.46,899.1)$ & $5,087.8(3,400.3 \quad 7,616.6)$ & $8 \%$ & $6,009.2(4,467.8$ 8,218.2) & $5,881.5(4,184.3 \quad 8,375.2)$ & $-2 \%$ \\
\hline Congenital anomalies & $130.9(93.7182 .5)$ & $141(101.2201 .8)$ & $8 \%$ & $1,278.3(1,065.61,416.6)$ & 778.7 (685.8 973.5) & $-39 \%$ \\
\hline Neural tube defects & $11.6(6.418 .7)$ & $8.2\left(\begin{array}{ll}4.4 & 13.6\end{array}\right)$ & $-29 \%$ & $99.4(58.5126 .5)$ & 18.5 (12.5 28) & $-81 \%$ \\
\hline Congenital heart anomalies & $34.2(17.666 .8)$ & $32.4(15.967 .4)$ & $-5 \%$ & $566.6(460.8650 .2)$ & $321.4(254.9411 .9)$ & $-43 \%$ \\
\hline Cleft lip and cleft palate & $5.4\left(\begin{array}{ll}2.7 & 9.7\end{array}\right)$ & $6(3.110 .5)$ & $12 \%$ & $7.9(4.912 .4)$ & $7.4(4.312 .1)$ & $-6 \%$ \\
\hline Down s syndrome & $38.1(21.661 .4)$ & $46.5(27.174 .6)$ & $22 \%$ & $104(69.3138 .5)$ & $104.7(80.1$ 136) & $1 \%$ \\
\hline Other chromosomal abnormalities & $14.5(8 \quad 23.9)$ & $17.4(10.129 .2)$ & $19 \%$ & $59.4(41.178 .5)$ & $55(39.277 .1)$ & $-7 \%$ \\
\hline Other congenital anomalies & $27.1(19.138 .3)$ & $30.6(21.844 .9)$ & $13 \%$ & 441 (294.4 539.7) & $271.6(215.5397 .8)$ & $-38 \%$ \\
\hline Skin and subcutaneous diseases & $1,989.9(1,235.63,086)$ & $2,206.8(1,347 \quad 3,476.9)$ & $11 \%$ & $2,058.2(1,3023,150.8)$ & $2,301.8(1,445 \quad 3,558.5)$ & $12 \%$ \\
\hline Eczema & $462.2(233.8802)$ & $511.7(252.5845 .6)$ & $11 \%$ & $462.2(233.8$ 802) & $511.7(252.5845 .6)$ & $11 \%$ \\
\hline Psoriasis & $87.3(42.4$ 145.8) & $105.1\left(\begin{array}{ll}50 & 172.9\end{array}\right)$ & $20 \%$ & $87.3(42.4$ 145.8) & $105.1\left(\begin{array}{ll}50 & 172.9\end{array}\right)$ & $20 \%$ \\
\hline Cellulitis & $11.1(1.245 .6)$ & $11(1.345 .3)$ & $-1 \%$ & $26.2(15.760 .2)$ & $29.6(16.963 .8)$ & $13 \%$ \\
\hline Abscess, impetigo, and other bacterial skin diseases & $41.8(16.985 .1)$ & $49.3(19.8101 .3)$ & $18 \%$ & $63.3(38$ 107.4) & $76(45.7$ 129.6) & $20 \%$ \\
\hline Scabies & $22.9(10.144 .9)$ & $26.2(11.849)$ & $15 \%$ & $22.9(10.144 .9)$ & $26.2(11.849)$ & $15 \%$ \\
\hline Fungal skin diseases & $107.6(33.8246 .6)$ & $128.2(39.3291 .8)$ & $19 \%$ & $107.6(33.8246 .6)$ & $128.2(39.3291 .8)$ & $19 \%$ \\
\hline Viral skin diseases & $189.2(72.5359 .4)$ & $182.5(66.3336 .4)$ & $-4 \%$ & $189.2(72.5359 .4)$ & $182.5(66.3336 .4)$ & $-4 \%$ \\
\hline Acne vulgaris & $312.6(139.6589 .8)$ & $237.6(106.6469 .3)$ & $-24 \%$ & $312.6(139.6589 .8)$ & $237.6(106.6469 .3)$ & $-24 \%$ \\
\hline Alopecia areata & $78.7(23158.4)$ & $91.1(25.1 \quad 182.4)$ & $16 \%$ & $78.7(23158.4)$ & $91.1(25.1$ 182.4) & $16 \%$ \\
\hline Pruritus & $179.4(82.5347 .4)$ & $244.2(110.2489 .4)$ & $36 \%$ & $179.4(82.5347 .4)$ & $244.2(110.2489 .4)$ & $36 \%$ \\
\hline Urticaria & $137.4(55.3236 .9)$ & $159\left(\begin{array}{ll}65 & 271\end{array}\right)$ & $16 \%$ & $137.4(55.3236 .9)$ & $159\left(\begin{array}{ll}65 & 271\end{array}\right)$ & $16 \%$ \\
\hline Decubitus ulcer & $30(9.869 .2)$ & $40.3(12.996 .9)$ & $34 \%$ & $61.5(39.8$ 101.6) & $89.7\left(\begin{array}{ll}55 & 150.1\end{array}\right)$ & $46 \%$ \\
\hline Other skin and subcutaneous diseases & $329.6(155.1$ 610.6) & 420.8 (193.8 806.2) & $28 \%$ & 329.8 (155.2 610.6) & 421 (193.9 806.3) & $28 \%$ \\
\hline
\end{tabular}


Table 3 Comparison of the main causes of disability-adjusted life years (DALYs) and years lived with disability (YLDs), in 1990 and 2010 , for the Spanish population (Continued)

\begin{tabular}{|c|c|c|c|c|c|c|}
\hline Sense organ diseases & 1637 (11642335) & $1,850.2(1291.12,656.9)$ & $13 \%$ & $1,637(1,1642,335)$ & $1,850.2(1,291.12,656.9)$ & $13 \%$ \\
\hline Glaucoma & $36(2354.9)$ & $56.9(37.682 .7)$ & $58 \%$ & $36(2354.9)$ & $56.9(37.682 .7)$ & $58 \%$ \\
\hline Cataracts & $193.1\left(\begin{array}{ll}136.8 & 261.7\end{array}\right)$ & $170.7(116.3233 .8)$ & $-12 \%$ & 193.1 (136.8 261.7) & 170.7 (116.3 233.8) & $-12 \%$ \\
\hline Macular degeneration & $58.4(37.587 .3)$ & $107.8(71.8152 .9)$ & $85 \%$ & $58.4(37.587 .3)$ & $107.8(71.8152 .9)$ & $85 \%$ \\
\hline Refraction and accommodation disorders & $84(61.3110 .7)$ & $107.9(77.6142 .3)$ & $28 \%$ & $84\left(\begin{array}{ll}61.3 & 110.7\end{array}\right)$ & $107.9(77.6142 .3)$ & $28 \%$ \\
\hline Other hearing loss & $926.8(541.41480 .3)$ & $962.7(554.5$ 1598.5) & $4 \%$ & $926.8(541.4 \quad 1,480.3)$ & $962.7(554.51,598.5)$ & $4 \%$ \\
\hline Other vision loss & $329.9(174.9586)$ & $433.2(222771)$ & $31 \%$ & $329.9(174.9586)$ & $433.2(222771)$ & $31 \%$ \\
\hline Other sense organ diseases & $8.8(3$ 19.8) & $11(3.725 .4)$ & $25 \%$ & $8.8(3 \quad 19.8)$ & $11(3.725 .4)$ & $25 \%$ \\
\hline Oral disorders & $942.4(564.3$ 1507.7) & 889.8 (519.4 1403.9) & $-6 \%$ & $942.4(564.31,507.7)$ & $889.8(519.4$ 1,403.9) & $-6 \%$ \\
\hline Dental caries & $110.2(45$ 216.1) & $130.4(52.8250 .1)$ & $18 \%$ & $110.2(45$ 216.1) & $130.4(52.8250 .1)$ & $18 \%$ \\
\hline Periodontal disease & $143(54.8312 .3)$ & $193.8(72.6400 .1)$ & $36 \%$ & $143\left(\begin{array}{lll}54.8 & 312.3\end{array}\right)$ & $193.8(72.6400 .1)$ & $36 \%$ \\
\hline Edentulism & $689.2(394.81103 .5)$ & $565.6(322903)$ & $-18 \%$ & $689.2(394.8$ 1103.5) & $565.6(322903)$ & $-18 \%$ \\
\hline Sudden infant death syndrome & $0\left(\begin{array}{ll}0 & 0\end{array}\right)$ & $0(0 \quad 0)$ & * & $93.3(40.6$ 150.8) & 61 (31.4 111.4) & $-35 \%$ \\
\hline Injuries & $3,873.3(2,679.95,500.9)$ & $5,495(3,846.67,727.1)$ & $42 \%$ & $11,961.1(10,559.1 \quad 13,749.9)$ & $9,962.5(8,154 \quad 12,170.7)$ & $-17 \%$ \\
\hline Transport injuries & $1,134(763.2 \quad 1,670.3)$ & $1,428.6(975$ 2,074.9) & $26 \%$ & $4,925.5(4,315.5$ 5,683.5) & $3,047.6(2,494.2 \quad 3,809.3)$ & $-38 \%$ \\
\hline Road injury & $982.9(659$ 1,450.2) & 1,197.1 (810.2 1,745.7) & $22 \%$ & $4,671.5(4,049.7 \quad 5,342.3)$ & $2,692.9(2,226.1 \quad 3,328.1)$ & $-42 \%$ \\
\hline Pedestrian injury by road vehicle & 169 (113.6 248.3) & $243.2(165350.8)$ & $44 \%$ & 580.3 (487.4 761.5) & $464.4(372.3584 .4)$ & $-20 \%$ \\
\hline Pedal cycle vehicle & 18.8 (12.7 27.8) & $50.5(34.274 .1)$ & $169 \%$ & $71.2(53.8110 .9)$ & $107.2(75.3136 .5)$ & $51 \%$ \\
\hline Motorized vehicle with two wheels & $232.4(155.6347 .3)$ & $172.6(116.1252 .7)$ & $-26 \%$ & $1,423.3(1,009.61,638.8)$ & $436(360.3539 .5)$ & $-69 \%$ \\
\hline Motorized vehicle with three or more wheels & $576.6(386.6852 .7)$ & $779.7(527.31134 .8)$ & $35 \%$ & $2,579.1(2,156.93,157.2)$ & $1,722.1(1,368.22,279.8)$ & $-33 \%$ \\
\hline Road injury other & $13(8.819 .2)$ & $12.1(8.217 .4)$ & $-7 \%$ & $44.6(31.760)$ & $24(17.733 .7)$ & $-46 \%$ \\
\hline Other transport injury & $151.1(102.4223)$ & $231.5(157.9331 .2)$ & $53 \%$ & $254(189.8359 .6)$ & $354.8(275.4460 .4)$ & $40 \%$ \\
\hline Unintentional injuries other than transport injuries & $2,638(1,821 \quad 3,727.8)$ & $3,944(2,760.55,515.8)$ & $50 \%$ & $5,486.5(4,503.8 \quad 6,659)$ & $5,472.7(4,205.37,082.7)$ & $0 \%$ \\
\hline Falls & $2,150.2(1,471.23043 .3)$ & $3,315.8(2,316.64,644.3)$ & $54 \%$ & $2,462.8(1,754.8 \quad 3,385.5)$ & $3,763.4(2,726.7 \quad 5,125)$ & $53 \%$ \\
\hline Drowning & $9.7(6.114 .5)$ & $11.1(7$ 16.8) & $14 \%$ & 404.4 (312.7 497.3) & $222.8\left(\begin{array}{ll}181 & 295.5\end{array}\right)$ & $-45 \%$ \\
\hline Fire, heat and hot substances & $57(30.5$ 106.3) & $70.2(37.6$ 127.1) & $23 \%$ & 158.1 (122.4 213) & $141\left(\begin{array}{ll}105.1202 .2\end{array}\right)$ & $-11 \%$ \\
\hline Poisonings & $9.2(5.614 .6)$ & $7.1(4.311 .3)$ & $-23 \%$ & 220.6 (153.6 338.5) & $165.9\left(\begin{array}{ll}79.7 & 233.9\end{array}\right)$ & $-25 \%$ \\
\hline Exposure to mechanical forces & $72.6\left(\begin{array}{ll}45.5 & 114\end{array}\right)$ & $75.2(47.8$ 113.9) & $4 \%$ & 269.1 (212.6 366.4) & $185.4(130.8252 .4)$ & $-31 \%$ \\
\hline Mechanical forces (firearm) & $26.9\left(\begin{array}{ll}17 & 42.2\end{array}\right)$ & $14.6(9.422 .2)$ & $-46 \%$ & $94.8\left(\begin{array}{ll}56.5 & 123\end{array}\right)$ & $31.6(22.747 .7)$ & $-67 \%$ \\
\hline Mechanical forces (other) & $56.5(35.488 .2)$ & $79.1(50.2$ 119.5) & $40 \%$ & $185.1(147.6282 .1)$ & 172.3 (131.7 241.7) & $-7 \%$ \\
\hline Adverse effects of medical treatment & $45.3(29.268 .3)$ & $102.6(66151.3)$ & $127 \%$ & 143.5 (115.6 181.7) & $227.3(174.5307 .9)$ & $58 \%$ \\
\hline Animal contact & $21.9\left(\begin{array}{ll}14.2 & 32.8)\end{array}\right.$ & $11.7\left(\begin{array}{ll}7.7 & 17.2\end{array}\right)$ & $-47 \%$ & $35.3(26.247 .7)$ & $18.6(14 \quad 25.1)$ & $-47 \%$ \\
\hline Animal contact (venomous) & $14.3(8.7 \quad 22.3)$ & $6.8(4.310 .5)$ & $-53 \%$ & $16.4(10.624 .8)$ & $8.3\left(\begin{array}{ll}5.6 & 12.2)\end{array}\right.$ & $-50 \%$ \\
\hline
\end{tabular}


Table 3 Comparison of the main causes of disability-adjusted life years (DALYs) and years lived with disability (YLDs), in 1990 and 2010 , for the Spanish population (Continued)

\begin{tabular}{|c|c|c|c|c|c|c|}
\hline Animal contact (non-venomous) & $7.6(4.811 .8)$ & $4.9(3 \quad 7.3)$ & $-36 \%$ & $18.9(13.924 .7)$ & $10.3\left(\begin{array}{ll}7.8 & 13.9)\end{array}\right.$ & $-45 \%$ \\
\hline Unintentional injuries not classified elsewhere & $272.1(185.1396 .4)$ & $350.3(232.7 \quad 505.7)$ & $29 \%$ & $1,792.7(1,054.8 \quad 2,031)$ & $748.2(605.7955 .4)$ & $-58 \%$ \\
\hline Self-harm and interpersonal violence & $101.3(66.6146 .4)$ & $122.3(81.1173)$ & $21 \%$ & $1,549.1(1,277.2$ 2,016.4) & $1,442.2(1,051.7 \quad 1,690.9)$ & $-7 \%$ \\
\hline Self-harm & $12.6(7.918 .3)$ & $15.2\left(\begin{array}{ll}9.7 & 23.1\end{array}\right)$ & $21 \%$ & 1,244.6 $(9,62.4 \quad 1,686.8)$ & $1,154.4(7,59.7 \quad 1,377.5)$ & $-7 \%$ \\
\hline Interpersonal violence & 88.7 (58.4 129.4) & $107.1(70.8151 .4)$ & $21 \%$ & 304.5 (241.9 403) & 287.8 (230.5 369.8) & $-5 \%$ \\
\hline Assault by firearm & $23.2\left(\begin{array}{ll}15 & 34.3\end{array}\right)$ & $30.1(19.542 .9)$ & $30 \%$ & $74.6(59105.3)$ & $74.2(57.398 .9)$ & $-1 \%$ \\
\hline Assault by sharp object & $31.9(20.946 .9)$ & $56.2(3779.6)$ & $76 \%$ & $106.7(79.1175 .7)$ & $143.2(94.8186 .6)$ & $34 \%$ \\
\hline Assault by other means & $40.1(26.557 .7)$ & $31.3(2143.8)$ & $-22 \%$ & $129.7(98.4158 .1)$ & 80.9 (65.5 106) & $-38 \%$ \\
\hline
\end{tabular}

Data are presented as mean values and uncertainty intervals (Lower, Upper).

* not applicable. 


\begin{tabular}{|c|c|c|c|c|}
\hline \multicolumn{2}{|r|}{1990 Main causes for DALYs } & \multicolumn{2}{|r|}{2010 Main causes for DALYS } & \multirow{2}{*}{$\begin{array}{r}\% \Delta \\
14 \%\end{array}$} \\
\hline 1 & Cardiovascular and circulatory diseases & 1 & Neoplasms & \\
\hline 2 & Neoplasms & 2 & Cardiovascular and circulatory diseases & $-11 \%$ \\
\hline 3 & Musculoskeletal disorders & 3 & Musculoskeletal disorders & $28 \%$ \\
\hline 4 & Mental and behavioral disorders & 4 & Mental and behavioraldisorders & $23 \%$ \\
\hline 5 & $\begin{array}{l}\text { Diabetes, urogenital, blood, and endocrine } \\
\text { diseases }\end{array}$ & 5 & $\begin{array}{l}\text { Diabetes, urogenital, blood, and endocrine } \\
\text { diseases }\end{array}$ & $20 \%$ \\
\hline 6 & Other non-communicable diseases & 6 & Neurological disorders & $61 \%$ \\
\hline 7 & Chronic respiratory diseases & 7 & Other non-communicable diseases & $-2 \%$ \\
\hline 8 & $\begin{array}{l}\text { Unintentional injuries other than transport } \\
\text { injuries }\end{array}$ & 8 & $\begin{array}{l}\text { Unintentional injuries other than transport } \\
\text { injuries }\end{array}$ & $-0,3 \%$ \\
\hline 9 & Transport injuries & 9 & Chronic respiratory diseases & $-6 \%$ \\
\hline 10 & Neurological disorders & 10 & Transport injuries & $-38 \%$ \\
\hline 11 & $\begin{array}{l}\text { Diarrhea, lower respiratory infections, } \\
\text { meningitis, and other common infectious } \\
\text { diseases }\end{array}$ & 11 & $\begin{array}{l}\text { Diarrhea, lower respiratory infections, } \\
\text { meningitis, and other common infectious } \\
\text { diseases }\end{array}$ & $-8 \%$ \\
\hline 12 & Cirrhosis of the liver & 12 & Digestive diseases (except cirrhosis) & $11 \%$ \\
\hline 13 & Digestive diseases (except cirrhosis) & 13 & Cirrhosis of the liver & $-25 \%$ \\
\hline 14 & Self-harm and interpersonal violence & 14 & Self-harm and interpersonal violence & $-7 \%$ \\
\hline 15 & HIV/AIDS and tuberculosis & 15 & Neonatal disorders & $-28 \%$ \\
\hline 16 & Neonatal disorders & 16 & HIV/AIDS and tuberculosis & $-52 \%$ \\
\hline 17 & Nutritional deficiencies & 17 & Nutritional deficiencies & $13 \%$ \\
\hline 18 & $\begin{array}{l}\text { Other communicable, maternal, neonatal, } \\
\text { and nutritional disorders }\end{array}$ & 18 & $\begin{array}{l}\text { Other communicable, maternal, neonatal, } \\
\text { and nutritional disorders }\end{array}$ & $8 \%$ \\
\hline 19 & Neglected tropical diseases and malaria & 19 & Neglected tropical diseases and malaria & $-7 \%$ \\
\hline 20 & Maternal disorders & 20 & Maternal disorders & $-22 \%$ \\
\hline
\end{tabular}

Figure 3 Spanish disability-adjusted life years (DALYs) ranks for the top 20 main causes in 1990 and 2010, and the percentage change between 1990 and 2010. Continuous line represents an ascending order in rank and the broken line represents a descending order. $(\Delta \%)=\%$ of change in absolute numbers of DALYs.
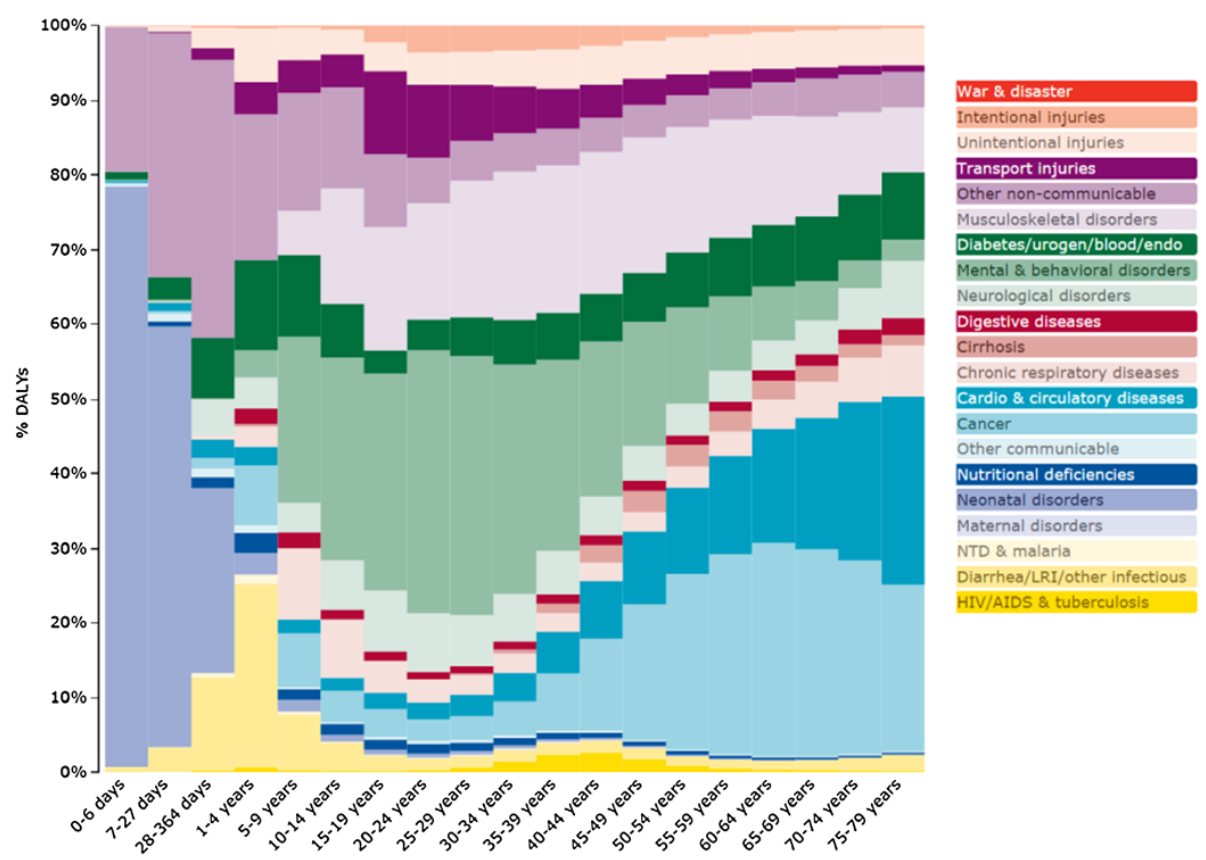

Figure 4 DALYs in Spain by cause and age in 2010. Adopted and modified from GBD data visualizations. 


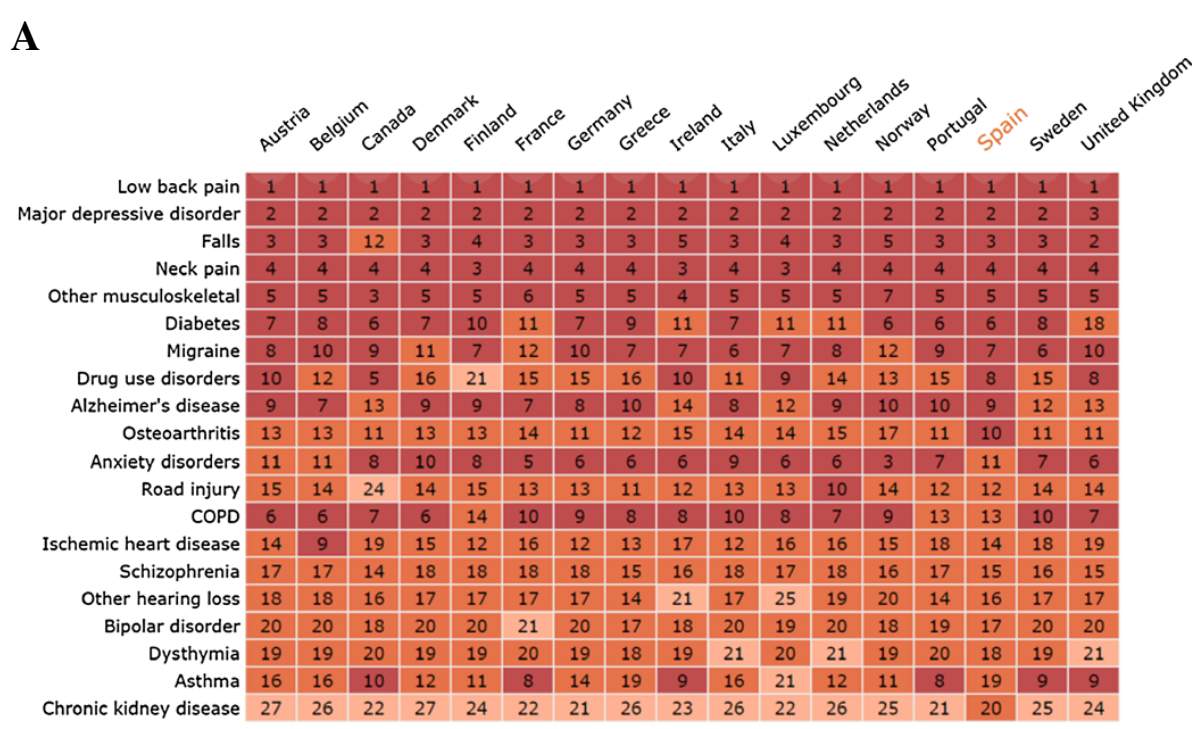

B

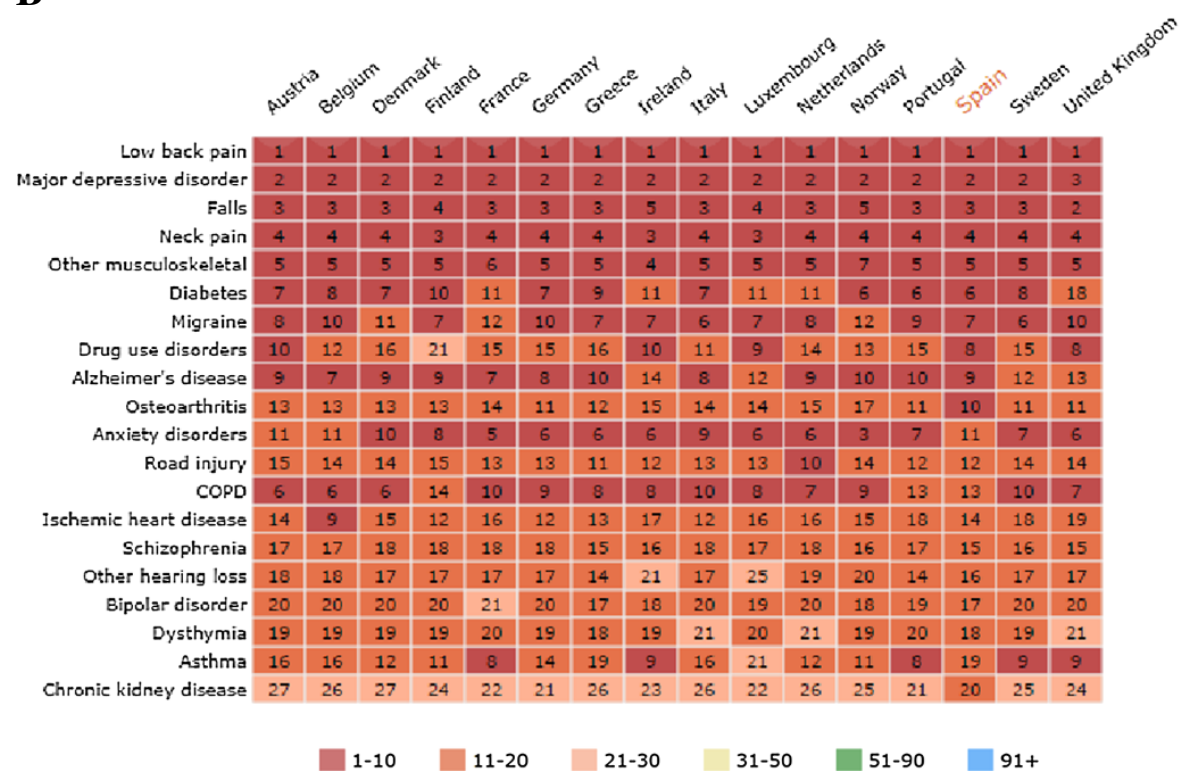

Figure 5 Age-standardized YLDs ranking relative to comparator European countries by the top 20 causes in (A) 1990 and (B) 2010. Numbers in cells indicate the ranks by country for each cause, with 1 being the disorder with the highest impact. The presented causes are ordered by the 20 leading causes of YLDs in Spain. YLDs, Years lived with disability; COPD, Chronic obstructive pulmonary disease. Adopted and modified from GBD data visualizations.

respiratory diseases by $6 \%$ (rank 9). Despite the population growth of $15 \%$ between 1990 and 2010 and the ageing of the population, the burden of disease increased only by $5 \%$. The analysis of changes by age and gender group denoted that DALYs decreased when analyzed as rates per 100,000 population.

The relevance of neoplasms and cardiovascular and circulatory diseases on population health is mostly driven by mortality. Accordingly, the cardiovascular and circulatory diseases, neoplasms, and injuries due to transport reasons were the top three leading causes for the burden of
YLLs. However, the impact of musculoskeletal disorders and mental disorders is mostly through YLDs, since they are the first and second causes in the ranking. Together, these two groups of disorders, accounted for almost the half (48\%) of all YLDs in Spain. Additionally, depression, and other mental disorders (like anxiety, etc.) have been associated to various musculoskeletal disorders [17,18]. All these conditions are related to occupational risk, particularly low back pain [19], and absenteeism. The impact of these disorders and the need for mental health promotion and musculoskeletal 
health prevention may have been underestimated by public health authorities and policies [20].

Other studies have previously evaluated the burden of morbidity and mortality in Spain $[8,9]$. According to these, in 2008, the major causes for mortality in males and females were also cardiovascular diseases and malignant neoplasms [8]. Furthermore, a recent study in Valencia reported similar results in mortality rates in the local population (i.e., $26 \%$ of all deaths due to malignant tumors and $34 \%$ due to cardiovascular diseases [9]).

Clear gender differences emerged in the analyses. Specifically, in males, cancer (i.e., neoplasms) was the main mortality cause followed by cardiovascular diseases, while in females the order was reversed. Risk factor differences may be causing the increased impact of cancer in males: they still have higher rates of smoking and heavy alcohol consumption [21,22]. The increased mortality of cardiovascular diseases in females is due to cerebro-vascular problems. Further, hormonal factors (disappearance of the protective role of estrogens after menopause) have been associated to the increased risk of stroke in females [23].

The top five causes for the burden of YLDs in Spain are similar to those in the other Mediterranean countries (except for slight differences with France) [24-26]. Similar results appeared also in the burden of DALYs comparing Spain to other European countries [24-26]. These similarities in the Mediterranean populations health could be a result of the common lifestyle habits (dietary habits, smoking, physical activity, etc.) [27], as well as shared genetic traits within the region [28]. Spain, as well as other Mediterranean countries, has to shift the provided health care services from curative to preventive $[29,30]$ and to identify the priority diseases for health research funding and prevention policies development [8].

When comparing the results among all European countries, some remarkable results have also been found. While there are substantial differences in the rank order of diseases regarding DALYs [7], there are much less differences in the first five causes of YLDs across European countries. Country differences may be mostly caused by differences in mortality instead of differences in YLDs. Whether this can be attributed to mortality figures being recorded more systematically in each country than prevalence figures should still be clarified.

In 2010, Spain presented the same leading conditions for YLLs compared to other Mediterranean countries, such as Greece, France, and Italy [24-26]. In 2010, Spain, France, and Italy showed age-standardized YLL rates for liver cancers significantly higher than the overall mean rate. Spain and Greece showed significantly higher rates of age-standardized YLL rates for bladder cancers [7]. Besides, the leading causes for premature mortality in the UK seem to be similar with those in Spain (i.e., cardiovascular and circulatory diseases and trachea, bronchus, and lung cancers [24-26]).

Globally, there is continuous shift from communicable to non-communicable diseases as the leading causes of mortality and DALYs [2,5]. According to the latest data for 2010, in the top ten leading causes of population $\mathrm{s}$ mortality, five were classified as non-communicable [2]. Global DALYs remained stable from 1990 to 2010 [5]. However, the global age-standardized DALY rates [5] actually decreased, which is consistent with our findings in Spain. Ischemic heart disease was the leading cause of DALYs worldwide in 2010, followed by lower respiratory infections, stroke, diarrheal diseases, and HIV/AIDS. Depressive disorders, also climbed from the fifteenth to the eleventh rank and road injury from twelfth to tenth rank $[2,5]$. However, in Spain the major causes for DALYs were neoplasms followed by cardiovascular diseases, musculoskeletal disorders, and mental disorders. Neurological disorders moved from the tenth to sixth rank; this high ranking in musculoskeletal disorders was quite similar with the global estimates [5] low back pain and neck pain are the most important contributors accompanied by osteoarthritis, rheumatoid arthritis, and gout [5,31]. The pathophysiology of chronic diseases, especially cardiovascular diseases and cancers, is complex, with the interaction of new environmental risk factors (e.g., multimorbidity, financial status, etc.), in addition to the classical modifiable risk factors (i.e., cognition reserve, smoking, unhealthy diet, physical activity, hypertension, etc.), making the prevention of chronic diseases in older adults quite difficult. Efforts to improve and protect health, prevent disease and injury, and deliver high-quality health care to the population must be tailored to address the causes associated with the greatest burden mainly of chronic diseases [32]. It is expected that preventive strategies can influence many of the aforementioned chronic conditions and non-communicable causes and increase the quality of life, while averting or minimizing the need for expensive medical care $[33,34]$. Furthermore, the engagement with the GBD collaborative group will provide more and better analysis data of the global burden of disease and, specifically, in Spain a fact that will contribute to continuous improvements of the health estimates in future iterations of the GBD study.

\section{Limitations}

The previous results should be considered taking into account the following limitations. Although Spain follows the European Statistics Code of Practice of 2006 2008 and data is collected in a consistent way across the country, there are a number of issues that should be considered when analyzing the results. Regarding mortality, the GBD project defined a number of garbage codes, which are causes of death that should not be identified as 
underlying causes of death but have been entered as the underlying causes of death on death certificates. Garbage codes were substituted by underlying causes based on pathophysiology. The fraction of garbage codes in Spain was within the world average. The substitution of garbage codes has impact on mortality causes. For example, in 2010, transport injury deaths based on raw data from Spain were 2,257. After correction for garbage codes and other adjustments, this number increased to 3,657 deaths. Following the modeling process, it further increased to 3,950 .

In this study, the calculation of DWs were based on surveys including several countries, and not just Spain. However, according to recent results [10], the DW assessments appeared to be consistent even among different cultural environments. Another aspect is the prevalence of the referred health conditions, which was based on epidemiological studies, a fact that may create uncertainty depending on the quality of the primary data. To overcome this issue, some expert groups developed tools to assess the risk of bias in the selected studies and sensitivity analyses were performed to weight study sizes according to their quality, or even to eliminate them from the final analysis [10]. As referred in previous works of the GBD study [10], Bayesian statistical models were used to estimate prevalence of conditions in each country by age, sex, and year. The nature of this estimation process implies that, in some cases, depending on the covariates, estimated variance might be smaller than the real variance across countries in a region, and in some other cases, uncertainty intervals for a specific estimate might be exaggerated. Furthermore, the calculation of uncertainty intervals throughout the Bayesian model analysis has provided some information on the extent of available information for Spain. However, the nature of the estimation process for causes of death and the prevalence of sequelae more generally lead to exaggerated uncertainty intervals in a high-income country such as Spain [12]. These wide uncertainty intervals may limit the number of the detected significant changes in the burden of disease between 1990 and 2010.

\section{Conclusions}

The present findings, together with previous work [5], suggest that cardiovascular and circulatory diseases, neoplasms, and mental and neurological disorders seem to be the leading causes of mortality as well as for YLDs and DALYs in Spain. Although several health promotion action plans have taken place globally $[35,36]$ in the past few years, morbidity throughout non-communicable diseases is increasing at alarming rates. Public health care systems have to focus further on the quality of health care services [37]. Furthermore, health promotion strategies should focus on health education programs that could improve quality of life. Public health care services have to shift from being curative to being preventive; in Spain, a strong emphasis should be given on health promotion, disease prevention, and rehabilitation.

\section{Abbreviations}

DALYs: Disability-adjusted life years; DW: Disability weight; GBD: Global

Burden of Diseases, Injuries, and Risk Factors; UK: United Kingdom;

YLDs: years lived with disability; YLLs: Years of life lost.

\section{Competing interests}

The authors declare that they have no competing interests.

\section{Authors contributions}

JMH and CJLM contributed to the concept and design of the study, acquisition of data, analysis and interpretation of data, and preparation of the manuscript. ST and NG contributed to the concept and design of the study, analysis and interpretation of data, and preparation of the manuscript. CDT, LC, LSR, and FPR contributed to interpretation of data, critical review and revision of the manuscript for important intellectual content. All authors read and approved the final manuscript.

\section{Acknowledgements}

Dr. Stefano Tyrovolas received a scholarship from the Foundation for Education and European Culture (IPEP) for his postdoctoral research, of which this work is part. Dr. Lidia Sanchez-Riera received a research grant from Sociedad Espaola de Reumatologa. Noe Garin is grateful to the FPU doctoral program (reference FPU12/05661) supported by the Spanish Ministry of Education, Culture and Sport.

\section{Source of funding}

Funding for GBD 2010 study was provided by the Bill \& Melinda Gates Foundation. This research was done as part of GBD 2010 study. All authors declare that they have no other funding, or any other support that could inappropriately influence the presented work.

\section{Author details}

'Parc Sanitari Sant Joan de Du, Universitat de Barcelona, Fundaci Sant Joan de Du. Dr Antoni Pujades, 42, 08830 Sant Boi de Llobregat, Barcelona, Spain. ${ }^{2}$ Instituto de Salud Carlos III, Centro de Investigacin Biomdica en Red de Salud Mental CIBERSAM, Dr. Esquerdo 46, 28007 Madrid, Spain. ${ }^{3}$ Unitat de Reumatologia. Hospital de la Santa Creu i Sant Pau, Universitat Autnoma de Barcelona, Carrer de Sant Quint, 89, 08026 Barcelona, Spain. ${ }^{4}$ Instituto de Salud Musculoesqueltica (InMusc), Calle Hilarin, Eslava 2 28015, Madrid, Spain. ${ }^{5}$ Institute of Bone and Joint Research, Royal North Shore Hospital, University of Sydney, Pacific Hwy, St Leonards, New South Wales 2065, Australia. ${ }^{6}$ Institut d Investigaci Biomdica de Bellvitge, Hospital Universitari de Bellvitge, Freixa Llarga s/n, 08907 L Hospitalet de Llobregat, Barcelona, Spain. ${ }^{7}$ Rheumatology Division, Cruces University Hospital, Crystal-induced arthritis investigation team, BioCruces Health Investigation Institute, Plaza de Cruces 12, 48903 Baracaldo, Vizcaya, Spain. ${ }^{8}$ Institute for Health Metrics and Evaluation, University of Washington, 2301 Fifth Avenue, mSuite 600, Seattle, WA 98121, USA.

Received: 2 July 2014 Accepted: 12 November 2014 Published online: 05 December 2014

References

1. Murray CJ, Salomon JA, Mathers CD, Lopez AD: Summary Measures of Population Health: Concepts, Ethics, Measurement and Applications. Geneva: WHO; 2002

2. Lozano R, Naghavi M, Foreman K, Lim S, Shibuya K, Aboyans V, Abraham J, Adair T, Aggarwal R, Ahn SY, Alvarado M, Anderson HR, Anderson LM, Andrews KG, Atkinson C, Baddour LM, Barker-Collo S, Bartels DH, Bell ML, Benjamin EJ, Bennett D, Bhalla K, Bikbov B, Bin Abdulhak A, Birbeck G, Blyth F, Bolliger I, Boufous S, Bucello C, Burch M, et al: Global and regional mortality from 235 causes of death for 20 age groups in 1990 and 2010: a systematic analysis for the global burden of disease study 2010. Lancet 2012, 380:2095 2128 
3. Murray CJ, Lopez AD: Mortality by cause for eight regions of the world: global burden of disease study. Lancet 1997, 349:1269 1276

4. Bank W, World Development Report 1993: Investing in Health. New York: Oxford University Press; 1993

5. Murray CJ, Vos T, Lozano R, Naghavi M, Flaxman AD, Michaud C, Ezzati M, Shibuya K, Salomon JA, Abdalla S, Aboyans V, Abraham J, Ackerman I, Aggarwal R, Ahn SY, Ali MK, Alvarado M, Anderson HR, Anderson LM, Andrews KG, Atkinson C, Baddour LM, Bahalim AN, Barker-Collo S, Barrero LH, Bartels DH, Basez MG, Baxter A, Bell ML, Benjamin EJ, et al: Disabilityadjusted life years (DALYs)for 291 diseases and injuries in 21 regions, 1990 2010: a systematic analysis for the global burden of disease study 2010. Lancet 2012, 380:21972223.

6. Murray CJ, Ezzati M, Flaxman AD, Lim S, Lozano R, Michaud C, Naghavi M, Salomon JA, Shibuya K, Vos T, Wikler D, Lopez AD: GBD 2010: design, definitions, and metrics. Lancet 2012, 380:2063 2066

7. Murray CJ, Richards MA, Newton JN, Fenton KA, Anderson HR, Atkinson C, Bennett D, Bernab E, Blencowe H, Bourne R, Braithwaite T, Brayne C, Bruce NG, Brugha TS, Burney P, Dherani M, Dolk H, Edmond K, Ezzati M, Flaxman $A D$, Fleming TD, Freedman G, Gunnell D, Hay RJ, Hutchings SJ, Ohno SL, Lozano R, Lyons RA, Marcenes W, Naghavi M, et al: UK health performance: findings of the global burden of disease study 2010. Lancet 2013, 381:997 1020.

8. Gnova-Maleras R, Ivarez-Martn E, Morant-Ginestar C, Fernndez de Larrea-Baz N, Catal-Lpez F: Measuring the burden of disease and injury in Spain using disability-adjusted life years: an updated and policyoriented overview. Public Health 2012, 126:1024 1031.

9. Catal-Lpez F, Gnova-Maleras R, Ridao M, Alvarez E, Sanflix-Gimeno G, Morant C, Peir S: Burden of disease assessment with summary measures of population health for the Region of Valencia, Spain: a populationbased study. Med Clin (Barc) 2013, 140:343 350

10. Vos T, Flaxman AD, Naghavi M, Lozano R, Michaud C, Ezzati M, Shibuya K, Salomon JA, Abdalla S, Aboyans V, Abraham J, Ackerman I, Aggarwal R, Ahn SY, Ali MK, Alvarado M, Anderson HR, Anderson LM, Andrews KG, Atkinson C, Baddour LM, Bahalim AN, Barker-Collo S, Barrero LH, Bartels DH, Basez MG, Baxter A, Bell ML, Benjamin EJ, Bennett D, et al: Years lived with disability (YLDs) for 1160 sequelae of 289 diseases and injuries 1990 2010: a systematic analysis for the global burden of disease study 2010. Lancet 2012, 380:2163 2196.

11. Wang H, Dwyer-Lindgren L, Lofgren KT, Rajaratnam JK, Marcus JR, LevinRector A, Levitz CE, Lopez AD, Murray CJ: Age-specific and sex-specific mortality in 187 countries, 1970 2010: a systematic analysis for the global burden of disease study 2010. Lancet 2012, 380:2071 2094.

12. Granados JA: Health at advanced age: social inequality and other factors potentially impacting longevity in nine high-income countries. Maturitas 2013, 74:137 147

13. Ahern RM, Lozano R, Naghavi M, Foreman K, Gakidou E, Murray CJ: Improving the public health utility of global cardiovascular mortality data: the rise of ischemic heart disease. Popul Health Metr 2011, 9:8.

14. Mathers CD, Vos ET, Stevenson CE, Begg SJ: The burden of disease and injury in Australia. Bull World Health Organ 2001, 79:1076 1084

15. Salomon JA, Vos T, Hogan DR, Gagnon M, Naghavi M, Mokdad A, Begum N, Shah R, Karyana M, Kosen S, Farje MR, Moncada G, Dutta A, Sazawal S, Dyer A, Seiler J, Aboyans V, Baker L, Baxter A, Benjamin EJ, Bhalla K, Bin Abdulhak A, Blyth F, Bourne R, Braithwaite T, Brooks P, Brugha TS, Bryan-Hancock C, Buchbinder R, Burney $P$, et al: Common values in assessing health outcomes from disease and injury: disability weights measurement study forthe Global Burden of Disease Study 2010. Lancet 2012, 380:2129 2143.

16. Ahmad OB, Boschi-Pinto C, Lopez AD, Murray CJ, Lozano R, Inoue M: Age Standardization of Rates: A New WHO Standard; 2001. [http://www.who. int/healthinfo/paper31.pdf]

17. Linton SJ: A review of psychological risk factors in back and neck pain. Spine 2000, 25:1148 1156.

18. Antonopoulou MD, Alegakis AK, Hadjipavlou AG, Lionis CD: Studying the association between musculoskeletal disorders, quality of life and mental health. A primary care pilot study in rural Crete, Greece. BMC Musculoskelet Disord 2009, 10:143.

19. Driscoll T, Jacklyn G, Orchard J, Passmore E, Vos T, Freedman G, Lim S, Punnett $L$ : The global burden of occupationally related low back pain: estimates from the Global Burden of Disease 2010 study. Ann Rheum Dis 2014, 73:975 981 .
20. Min JA, Lee CU, Lee C: Mental health promotion and illness prevention: a challenge for psychiatrists. Psychiatry Investig 2013, 10:307 316.

21. Mashberg A, Boffetta P, Winkelman R, Garfinkel L: Tobacco smoking, alcohol drinking, and cancer of the oral cavity and oropharynx among U.S. veterans. Cancer 1993, 72:1369 1375

22. Rota M, Pasquali E, Bellocco R, Bagnardi V, Scotti L, Islami F, Negri E, Boffetta P, Pelucchi C, Corrao G, La Vecchia C: Alcohol drinking and cutaneous melanoma risk: a systematic review and dose-risk meta-analysis. Br J Dermatol. 2014, 170:1021 1108

23. Haast RA, Gustafson DR, Kiliaan AJ: Sex differences in stroke. J Cereb Blood Flow Metab 2012, 32:2100 2107.

24. Institute for Health Metrics and Evaluation: GBD Profile: France. [http:// www.healthdata.org/sites/default/files/files/country_profiles/GBD/ inme_gbd_country_report_france.pdf]

25. Institute for Health Metrics and Evaluation: GBD Profile: Greece. [http:// www.healthdata.org/sites/default/files/files/country_profiles/GBD/ inme_gbd_country_report_greece.pdf]

26. Institute for Health Metrics and Evaluation: GBD Profile: Italy. [http://www. healthdata.org/sites/default/files/files/country_profiles/GBD/

inme_gbd_country_report_italy.pdf]

27. Tourlouki E, Matalas AL, Bountziouka V, Tyrovolas S, Zeimbekis A, Gotsis E, Tsiligianni I, Protopapa I, Protopapas C, Metallinos G, Lionis C, Piscopo S, Polychronopoulos E, Panagiotakos DB: Are current dietary habits in Mediterranean islands a reflection of the past? Results from the MEDIS study. Ecol Food Nutr 2013, 52:371 386.

28. Athanasiadis G, Gonzlez-Prez E, Esteban E, Dugoujon JM, Stoneking M, Moral P: The Mediterranean Sea as a barrier to gene flow: evidence from variation in and around the F7 and F12 genomic regions. BMC Evol Biol 2010, 10:84

29. Spanish Ministry of Health, Social Policy and Equality: Ischaemic heart disease strategy of the Spanish National Health System. Madrid: Spanish Ministry of Health, Social Policy and Equality; 2009. [http://www.msc.es/ organizacion/sns/planCalidadSNS/docs/Summary_Ischaemic_Heart_ Disease_Strategy_Spanish_National_Health_System.pdf]

30. Spanish Ministry of Health, Social Policy and Equality: Mental health strategy of the Spanish National Health System. Madrid: Spanish Ministry of Health, Social Policy and Equality; 2011. [http://www.msps.es/organizacion/sns/ planCalidadSNS/docs/saludmental/SaludMental2009-2013.pdf]

31. Hoy D, March L, Brooks P, Blyth F, Woolf A, Bain C, Williams G, Smith E, Vos $T$, Barendregt J, Murray C, Burstein R, Buchbinder R: The global burden of low back pain: estimates from the global burden of disease 2010 study. Ann Rheum Dis 2014, 73:968 974.

32. Hopman WM, Harrison MB, Coo H, Friedberg E, Buchanan M, VanDenKerkhof EG: Associations between chronic disease, age and physical and mental health status. Chronic Dis Can 2009, 29:108 116

33. Starfield B, Shi L, Macinko J: Contribution of primary care to health systems and health. Milbank Q 2005, 83:457 502.

34. Panagiotakos DB, Pitsavos C, Chrysohoou C, Skoumas I, Stefanadis C: Prevalence and five-year incidence (2001 2006) of cardiovascular disease risk factors in a Greek sample: the ATTICA study. Hellenic J Cardiol 2009, 250:388 395

35. World Health Organization: Active Ageing: A Policy Framework. Geneva: WHO; 2002. [http://whqlibdoc.who.int/hq/2002/WHO_NMH_NPH_02.8.pdf]

36. World Health Organization: Global Strategy on Diet, Physical Activity and Health. Geneva: WHO; 2004

37. World Health Organization: Strengthening Public Health Services Across the European Region A Summary of Background Documents for the European Action Plan. Denmark: WHO Regional Office for Europe; 2012. [http://www.euro.who.int/_data/assets/pdf_file/0017/172016/RC62-id05final-Eng.pdf]

doi:10.1186/s12916-014-0236-9

Cite this article as: Haro et al:: The burden of disease in Spain: results from the global burden of disease study 2010. BMC Medicine 2014 12:236. 